\title{
Preparation and Photophysical Properties of
}

\author{
Bis(tridentate) Iridium(III) Emitters: Pincer
}

\section{Coordination of 2,6-Di(2-pyridyl)phenyl}

Pierre-Luc T. Boudreault, ${ }^{b}$ Miguel A. Esteruelas, ${ }^{*}$, Daniel Gómez-Bautista, ${ }^{a}$ Susana Izquierdo, ${ }^{a}$ Ana M. López, ${ }^{a}$ Enrique Oñate, ${ }^{a}$ Esther Raga, ${ }^{a}$ Jui-Yi Tsai ${ }^{b}$

${ }^{a}$ Departamento de Química Inorgánica - Instituto de Síntesis Química y Catálisis Homogénea (ISQCH) - Centro de Innovación en Química Avanzada (ORFEO-CINQA), Universidad de Zaragoza - CSIC, 50009 Zaragoza, Spain

${ }^{b}$ Universal Display Corporation, 375 Phillips Boulevard, Ewing, New Jersey 08618, USA 


\section{Contents:}

$\begin{array}{lr}\text { Experimental Section: General Information } & \text { S3 }\end{array}$

$\begin{array}{ll}\text { Structural Analysis of Complexes 2, 3, 6, 7, and } 9 & \text { S3 }\end{array}$

$\begin{array}{ll}\text { Computational Details } & \text { S5 }\end{array}$

$\begin{array}{lr}\text { Energies of Optimized Structures } & \text { S5 }\end{array}$

UV-vis Spectra of Complexes 4-7 and 9 (Observed and Calculated) S8

Analysis of Computed UV/Vis Data for 4-7 and 9 10

$\begin{array}{lr}\text { Cyclic Voltammograms of Complexes 4-7 and } 9 & \text { S15 }\end{array}$

Theoretical Analysis of Molecular Orbitals of Complexes 4-7 and $9 \quad$ S16

Normalized Excitation and Emission Spectra of Complexes 4-7 and $9 \quad$ S27

$\begin{array}{lr}\text { NMR Spectra of Complexes 2-9 } & \text { S32 }\end{array}$

TGA Curves of Complexes 4-7 and 9 S50

$\begin{array}{lr}\text { DSC Curves of Complexes } 6 \text { and } 7 & \text { S51 }\end{array}$

$\begin{array}{ll}\text { References } & \text { S52 }\end{array}$ 
Experimental Section: General Information. ${ }^{1} \mathrm{H},{ }^{31} \mathrm{P}\left\{{ }^{1} \mathrm{H}\right\}$, and ${ }^{13} \mathrm{C}\left\{{ }^{1} \mathrm{H}\right\}$ NMR spectra were recorded on Bruker Avance 300 or $400 \mathrm{MHz}$ instrument. C, H, and $\mathrm{N}$ analyses were carried out in a Perkin-Elmer $2400 \mathrm{CHNS} / \mathrm{O}$ analyzer. High-resolution electrospray mass spectra were acquired using a MicroTOF-Q hybrid quadrupole time-of-flight spectrometer (Bruker Daltonics, Bremen, Germany). UV-visible spectra were registered on an Evolution 600 spectrophotomer. Steady-state photoluminescence spectra were recorded on a Jobin-Yvon Horiba Fluorolog FL-3-11 spectrofluorimeter. Lifetimes were measured using an IBH 5000F coaxial nanosecond flash lamp. Quantum yields were measured using the Hamamatsu Absolute PL Quantum Yield Measurement System C11347-11. Cyclic voltammetry measurements were performed using a Voltalab PST050 potentiostat with Pt wire as working electrode, Pt wire as counter electrode, and saturated calomel (SCE) as reference electrode. The experiments were carried out under argon in dichloromethane or acetonitrile solutions $\left(10^{-3} \mathrm{M}\right)$, with $\mathrm{Bu}_{4} \mathrm{NPF}_{6}$ as supporting electrolyte $(0.1 \mathrm{M})$. Scan rate was 100 $\mathrm{mV} \cdot \mathrm{s}^{-1}$. The potentials were referenced to the ferrocene/ferrocenium $\left(\mathrm{Fc} / \mathrm{Fc}^{+}\right)$couple.

Structural Analysis of Complexes 2, 3, 6, 7, and 9. X-ray data were collected on a Bruker Smart APEX or Bruker Smart APEX DUO CCD diffractometers equipped with a normal focus, and $2.4 \mathrm{~kW}$ sealed tube source (Mo radiation, $\lambda=0.71073 \AA$ ). Data were collected over the complete sphere covering $0.3^{\circ}$ in $\omega$. Data were corrected for absorption by using a multiscan method applied with the SADABS program. ${ }^{1}$ The structures were solved by Patterson or direct methods and refined by fullmatrix least squares on $\mathrm{F}^{2}$ with SHELXL2016, ${ }^{2}$ including isotropic and subsequently anisotropic displacement parameters. The hydrogen atoms were observed in the last Fourier Maps or calculated, and refined freely or using a restricted riding model.

Crystals of $\mathbf{3}$ were obtained as twins that could not be completely solved. As a result the most intense residual peak is relatively high $\left(14.5 \mathrm{e} / \AA^{3}\right)$ and its position is related to that of the iridium atom $(\mathrm{x}, \mathrm{y}, \mathrm{z}+0.5)$.

Crystal data for 2 (CCDC 1968299): $\mathrm{C}_{18} \mathrm{H}_{17} \mathrm{Cl}_{2} \mathrm{IrN}_{2} \mathrm{OS}, \mathrm{C}_{2} \mathrm{H}_{6} \mathrm{OS}, \mathrm{M}_{\mathrm{W}}$ 650.62, yellow, irregular block $\left(0.101 \times 0.068 \times 0.057 \mathrm{~mm}^{3}\right)$, monoclinic, space group $\mathrm{P} 2{ }_{1} / \mathrm{n}, a: 10.3654(11) \AA, b: 14.9081(15)$ $\AA, c: 15.0397(16) \AA, \beta: 109.7090(10)^{\circ}, V=2187.9(4) \AA^{3}, Z=4, Z^{\prime}=1, D_{\text {calc }}: 1.975 \mathrm{~g} \mathrm{~cm}^{-3}, \mathrm{~F}(000)$ : 1264, $T=100(2) \mathrm{K}, \mu 6.558 \mathrm{~mm}^{-1} .33948$ measured reflections $\left(2 \theta: 3-57^{\circ}, \omega\right.$ scans $\left.0.3^{\circ}\right), 5835$ unique $\left(\mathrm{R}_{\text {int }}=0.0348\right)$; min./max. transm. Factors $0.704 / 0.746$. Final agreement factors were $\mathrm{R}^{1}=$ 0.0192 (5210 observed reflections, $\mathrm{I}>2 \sigma(\mathrm{I})$ ) and $\mathrm{wR}^{2}=0.0425$; data/restraints/parameters 5835/0/266; GoF = 1.042. Largest peak and hole: 0.909 (close to iridium atoms) and $-0.513 \mathrm{e} / \AA^{3}$.

Crystal data for 3 (CCDC 1968295): $\mathrm{C}_{24} \mathrm{H}_{25} \mathrm{Cl}_{2} \mathrm{IrN}_{2}, \mathrm{M}_{\mathrm{W}} 604.56$, yellow, irregular block $(0.160 \mathrm{x}$ $0.120 \times 0.080 \mathrm{~mm}^{3}$ ), monoclinic, space group $\mathrm{P} 21 / \mathrm{c}, a: 14.940(3) \AA, b: 10.148(2) \AA, c: 13.943(3) \AA$, 
$\beta: 90.767(4)^{\circ}, V=2113.6(8) \AA^{3}, Z=4, Z^{\prime}=1, D_{\text {calc }}: 1.900 \mathrm{~g} \mathrm{~cm}^{-3}, \mathrm{~F}(000): 1176, T=100(2) \mathrm{K}, \mu$ $6.583 \mathrm{~mm}^{-1} .16427$ measured reflections $\left(2 \theta: 3-51^{\circ}, \omega\right.$ scans $\left.0.3^{\circ}\right), 3766$ unique $\left(\mathrm{R}_{\mathrm{int}}=0.1181\right)$; min./max. transm. Factors 0.630/0.862. Final agreement factors were $\mathrm{R}^{1}=0.0724$ (2926 observed reflections, $\mathrm{I}>2 \sigma(\mathrm{I}))$ and $\mathrm{wR}^{2}=0.1766$; data/restraints/parameters 3766/18/263; GoF = 1.350. Largest peak and hole 14.498 and $-2.351 \mathrm{e} / \AA^{3}$.

Crystal data for 6 (CCDC 1968296): $\mathrm{C}_{32} \mathrm{H}_{24} \mathrm{IrN}_{5}, \mathrm{CH}_{4} \mathrm{O}, \mathrm{H}_{2} \mathrm{O}, \mathrm{M}_{\mathrm{W}}$ 720.82, orange, irregular block $\left(0.234 \times 0.083 \times 0.066 \mathrm{~mm}^{3}\right)$, triclinic, space group P-1, $a: 8.8148(10) \AA, b: 12.6168(15) \AA, c$ : $13.0145(15) \AA, \alpha: 87.249(2)^{\circ}, \beta: 76.4980(10)^{\circ}, \gamma: 87.665(2)^{\circ}, V=1405.1(3) \AA^{3}, Z=2, Z^{\prime}=1, D_{\text {calc: }}$ : $1.704 \mathrm{~g} \mathrm{~cm}^{-3}, \mathrm{~F}(000): 712, \mathrm{~T}=100(2) \mathrm{K}, \mu 4.791 \mathrm{~mm}^{-1} .16504$ measured reflections $\left(2 \theta: 3-57^{\circ}, \omega\right.$ scans $\left.0.3^{\circ}\right), 6587$ unique $\left(\mathrm{R}_{\mathrm{int}}=0.0482\right)$; min./max. transm. Factors $0.656 / 0.862$. Final agreement factors were $\mathrm{R}^{1}=0.0552$ (5169 observed reflections, $\mathrm{I}>2 \sigma(\mathrm{I})$ ) and $\mathrm{wR}^{2}=0.1319$; data/restraints/parameters 6587/6/363; GoF $=1.048$. Largest peak and hole 2.026 (close to iridium atoms) and $-1.639 \mathrm{e} / \AA^{3}$.

Crystal data for 7 (CCDC 1968297): $\mathrm{C}_{36} \mathrm{H}_{26} \mathrm{IrN}_{5}, 0.5\left(\mathrm{CH}_{2} \mathrm{Cl}_{2}\right), \mathrm{CH}_{4} \mathrm{O}, \mathrm{M}_{\mathrm{W}}$ 795.32, yellow, irregular block $\left(0.160 \times 0.139 \times 0.109 \mathrm{~mm}^{3}\right)$, monoclinic, space group P2 $1 / \mathrm{c}, a$ : 8.5953(4) $\AA, b$ : 12.2424(6) A, $c: 29.8095(15) \AA, \beta: 92.2540(10)^{\circ}, V=3134.3(3) \AA^{3}, Z=4, Z^{\prime}=1, D_{\text {calc }}: 1.685 \mathrm{~g} \mathrm{~cm}^{-}$ ${ }^{3}, \mathrm{~F}(000): 1572, T=100(2) \mathrm{K}, \mu 4.385 \mathrm{~mm}^{-1} .43536$ measured reflections $\left(2 \theta: 3-57^{\circ}, \omega\right.$ scans $0.3^{\circ}$ ), 7634 unique $\left(\mathrm{R}_{\text {int }}=0.0402\right)$; min./max. transm. Factors $0.654 / 0.862$. Final agreement factors were $\mathrm{R}^{1}$ $=0.0277(6563$ observed reflections, $\mathrm{I}>2 \sigma(\mathrm{I}))$ and $\mathrm{wR}^{2}=0.0629$; data/restraints/parameters $7634 / 36 / 428 ; \mathrm{GoF}=1.109$. Largest peak and hole 0.873 (close to iridium atoms) and $-1.027 \mathrm{e} / \AA^{3}$.

Crystal data for 9 (CCDC 1968298): $\mathrm{C}_{37} \mathrm{H}_{27} \mathrm{IrN}_{4}, \mathrm{CH}_{2} \mathrm{Cl}_{2}, \mathrm{M}_{\mathrm{W}}$ 804.75, yellow, irregular block $\left(0.300 \times 0.020 \times 0.020 \mathrm{~mm}^{3}\right)$, monoclinic, space group P2 $1 / \mathrm{c}, a: 8.7712(13) \AA, b: 14.172(2) \AA, c$ : 25.055(4) $\AA, \beta: 99.357(2)^{\circ}, V=3073.0(8) \AA^{3}, Z=4, Z^{\prime}=1, D_{\text {calc }}: 1.739 \mathrm{~g} \mathrm{~cm}^{-3}, \mathrm{~F}(000): 1584, \mathrm{~T}=$ $100(2) \mathrm{K}, \mu 4.555 \mathrm{~mm}^{-1} .47074$ measured reflections $\left(2 \theta: 3-57^{\circ}\right.$, $\omega$ scans $\left.0.3^{\circ}\right), 6175$ unique $\left(\mathrm{R}_{\text {int }}=\right.$ 0.1009); min./max. transm. Factors $0.706 / 0.862$. Final agreement factors were $R^{1}=0.0413(4767$ observed reflections, $\mathrm{I}>2 \sigma(\mathrm{I}))$ and $\mathrm{wR}^{2}=0.0783$; data/restraints/parameters 6175/0/408; GoF = 1.049. Largest peak and hole 1.408 (close to iridium atoms) and $-1.535 \mathrm{e} / \AA^{3}$ 
Computational Details. All calculations were performed at the DFT level using the B3LYP functional $^{3}$ supplemented with the Grimme's dispersion correction $\mathrm{D} 3^{4}$ as implemented in Gaussian09. ${ }^{5}$ Ir atoms were described by means of an effective core potential SDD for the inner electron $^{6}$ and its associated double- $\zeta$ basis set for the outer ones, complemented with a set of $f$ polarization functions for iridium. ${ }^{7}$ The $6-31 \mathrm{G}^{* *}$ basis set was used for the $\mathrm{H}, \mathrm{C}$, and $\mathrm{N}$ atoms. ${ }^{8}$ All minima were verified to have no negative frequencies. The geometries were fully optimized in vacuo and in THF $(\varepsilon=7.4257)$ solvent using the continuum SMD model. ${ }^{9}$ We performed TD-DFT calculations at the same level of theory in THF calculating the lowest 50 singlet-singlet excitations at the ground state $S_{0}$. It should be noted that the singlet-triplet excitations are set to zero due to the neglect of spin-orbit coupling in the TDDFT calculations as implemented in G09. The UV/vis absorption spectra were obtained by using the GaussSum 3 software. ${ }^{10}$ The phosphorescence emission compares well with the 0-0 transition calculated taking into account the zero point energies (zpe) of the geometries of both the optimized $\mathrm{T}_{1}$ and $\mathrm{S}_{0}$ states in THF $(\mathbf{4}, \mathbf{5}, \mathbf{6}, \mathbf{7})$ or the difference of energies of both the optimized $\mathrm{T}_{1}$ and the singlet with the same geometry (9).

\section{Energies of Optimized Structures}

\section{Complex $4\left(\mathrm{~S}_{0}, \mathrm{THF}\right)$}

$\begin{array}{lc}\text { Zero-point correction= } & \begin{array}{c}0.430295 \\ \text { (Hartree/Particle) }\end{array} \\ \text { Thermal correction to Energy= } & 0.456836 \\ \text { Thermal correction to Enthalpy }= & 0.457780 \\ \text { Thermal correction to Gibbs Free Energy }= & 0.373112 \\ \text { Sum of electronic and zero-point Energies }= & -1533.249178 \\ \text { Sum of electronic and thermal Energies }= & -1533.222638 \\ \text { Sum of electronic and thermal Enthalpies }= & -1533.221693 \\ \text { Sum of electronic and thermal Free Energies }= & -1533.306362\end{array}$

\section{Complex $4\left(T_{1}\right.$, THF $)$}

Zero-point correction=

Thermal correction to Energy=

Thermal correction to Enthalpy=

Thermal correction to Gibbs Free Energy=

Sum of electronic and zero-point Energies=

Sum of electronic and thermal Energies=

Sum of electronic and thermal Enthalpies=

Sum of electronic and thermal Free Energies=
0.425024 (Hartree/Particle)

0.452407

0.453351

0.365797

$-1533.164351$

$-1533.136968$

$-1533.136023$

$-1533.223578$ 


\section{Complex $5\left(S_{0}\right.$, THF $)$}

Zero-point correction=

Thermal correction to Energy=

Thermal correction to Enthalpy=
0.477447 (Hartree/Particle)

0.506506

0.507450

Thermal correction to Gibbs Free Energy $=\quad 0.418190$

Sum of electronic and zero-point Energies=

$-1686.866986$

Sum of electronic and thermal Energies=

$-1686.837927$

Sum of electronic and thermal Enthalpies=

$-1686.836983$

Sum of electronic and thermal Free Energies=

$-1686.926243$

\section{Complex $5\left(\mathrm{~T}_{1}, \mathrm{THF}\right)$}

Zero-point correction=

Thermal correction to Energy=

Thermal correction to Enthalpy=
0.472531 (Hartree/Particle)

0.502398

0.503342

Thermal correction to Gibbs Free Energy= 0.410966

Sum of electronic and zero-point Energies= $-1686.781912$

Sum of electronic and thermal Energies= $-1686.752045$

Sum of electronic and thermal Enthalpies= $-1686.751101$

Sum of electronic and thermal Free Energies=

$-1686.843477$

\section{Complex $6\left(\mathrm{~S}_{0}, \mathrm{THF}\right)$}

Zero-point correction=

Thermal correction to Energy=

Thermal correction to Enthalpy=
0.486244 (Hartree/Particle)

0.515764

0.516708

Thermal correction to Gibbs Free Energy $=\quad 0.426400$

Sum of electronic and zero-point Energies= $\quad-1611.836054$

Sum of electronic and thermal Energies $=\quad-1611.806534$

Sum of electronic and thermal Enthalpies= $\quad-1611.805590$

Sum of electronic and thermal Free Energies $=\quad-1611.895898$

\section{Complex $6\left(T_{1}\right.$, THF $)$}

Zero-point correction=

Thermal correction to Energy=

Thermal correction to Enthalpy=
0.482331 (Hartree/Particle)

0.512370

0.513315

Thermal correction to Gibbs Free Energy= 0.421434

Sum of electronic and zero-point Energies= $-1611.747923$

Sum of electronic and thermal Energies= $-1611.717883$

Sum of electronic and thermal Enthalpies= $-1611.716939$

Sum of electronic and thermal Free Energies= $-1611.808819$

\section{Complex $7\left(S_{0}\right.$, THF $)$}




$\begin{array}{lc}\text { Thermal correction to Energy= } & 0.565438 \\ \text { Thermal correction to Enthalpy= } & 0.566382 \\ \text { Thermal correction to Gibbs Free Energy= } & 0.469366 \\ \text { Sum of electronic and zero-point Energies }= & -1765.454255 \\ \text { Sum of electronic and thermal Energies }= & -1765.421998 \\ \text { Sum of electronic and thermal Enthalpies }= & -1765.421054 \\ \text { Sum of electronic and thermal Free Energies }= & -1765.518070\end{array}$

\section{Complex $7\left(T_{1}\right.$, THF $)$}

$\begin{array}{lcc}\text { Zero-point correction }= & 0.528689 \text { (Hartree/Particle) } \\ \text { Thermal correction to Energy= } & 0.561641 \\ \text { Thermal correction to Enthalpy= } & 0.562585 \\ \text { Thermal correction to Gibbs Free Energy= } & 0.463168 \\ \text { Sum of electronic and zero-point Energies }= & -1765.366701 \\ \text { Sum of electronic and thermal Energies }= & -1765.333750 \\ \text { Sum of electronic and thermal Enthalpies }= & -1765.332805 \\ \text { Sum of electronic and thermal Free Energies }= & -1765.432222\end{array}$

\section{Complex $9\left(S_{0}\right.$, THF)}

$\begin{array}{lc}\text { Zero-point correction= } & 0.544126 \text { (Hartree/Particle) } \\ \text { Thermal correction to Energy= } & 0.576536 \\ \text { Thermal correction to Enthalpy= } & 0.577480 \\ \text { Thermal correction to Gibbs Free Energy= } & 0.481043 \\ \text { Sum of electronic and zero-point Energies }= & -1749.388260 \\ \text { Sum of electronic and thermal Energies }= & -1749.355850 \\ \text { Sum of electronic and thermal Enthalpies }= & -1749.354906 \\ \text { Sum of electronic and thermal Free Energies }= & -1749.451344\end{array}$

\section{Complex $9\left(T_{1}\right.$, THF $)$}

E(RB3LYP)

Zero-point correction=

Thermal correction to Energy=

Thermal correction to Enthalpy=

Thermal correction to Gibbs Free Energy=

Sum of electronic and zero-point Energies=

Sum of electronic and thermal Energies=

Sum of electronic and thermal Enthalpies=

Sum of electronic and thermal Free Energies=

0.539090 (Hartree/Particle)

0.572239

0.573183

0.473812

$-1749.303747$

$-1749.270598$

$-1749.269654$

$-1749.369025$

\section{Complex 9 (S structure of $T_{1}, T H F$ )}

E(RB3LYP)

-1749.92421591 a.u. 

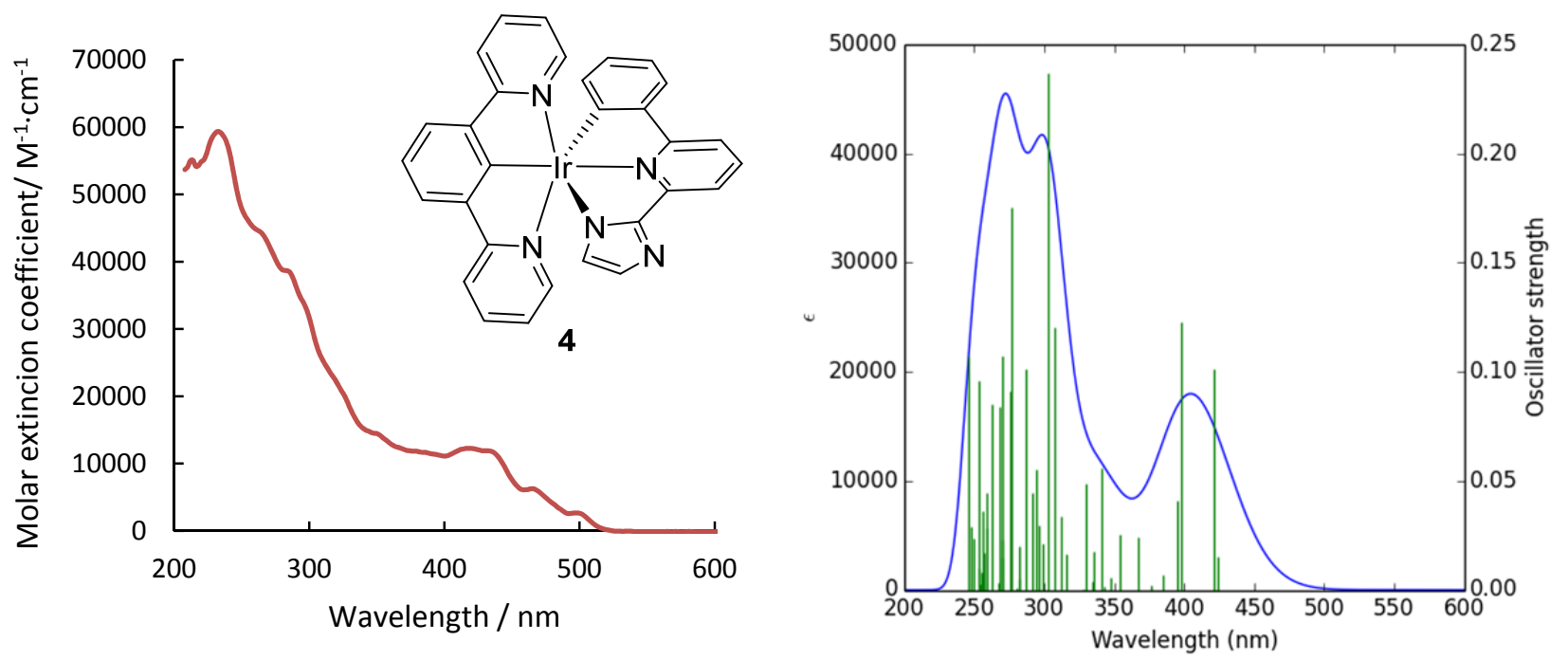

Figure S1. Observed UV-vis spectrum of complex 4 in 2-MeTHF $\left(2.0 \times 10^{-5} \mathrm{M}\right)$ and calculated (B3LYP(GD3)//SDD(f)/6-31G**) in THF.
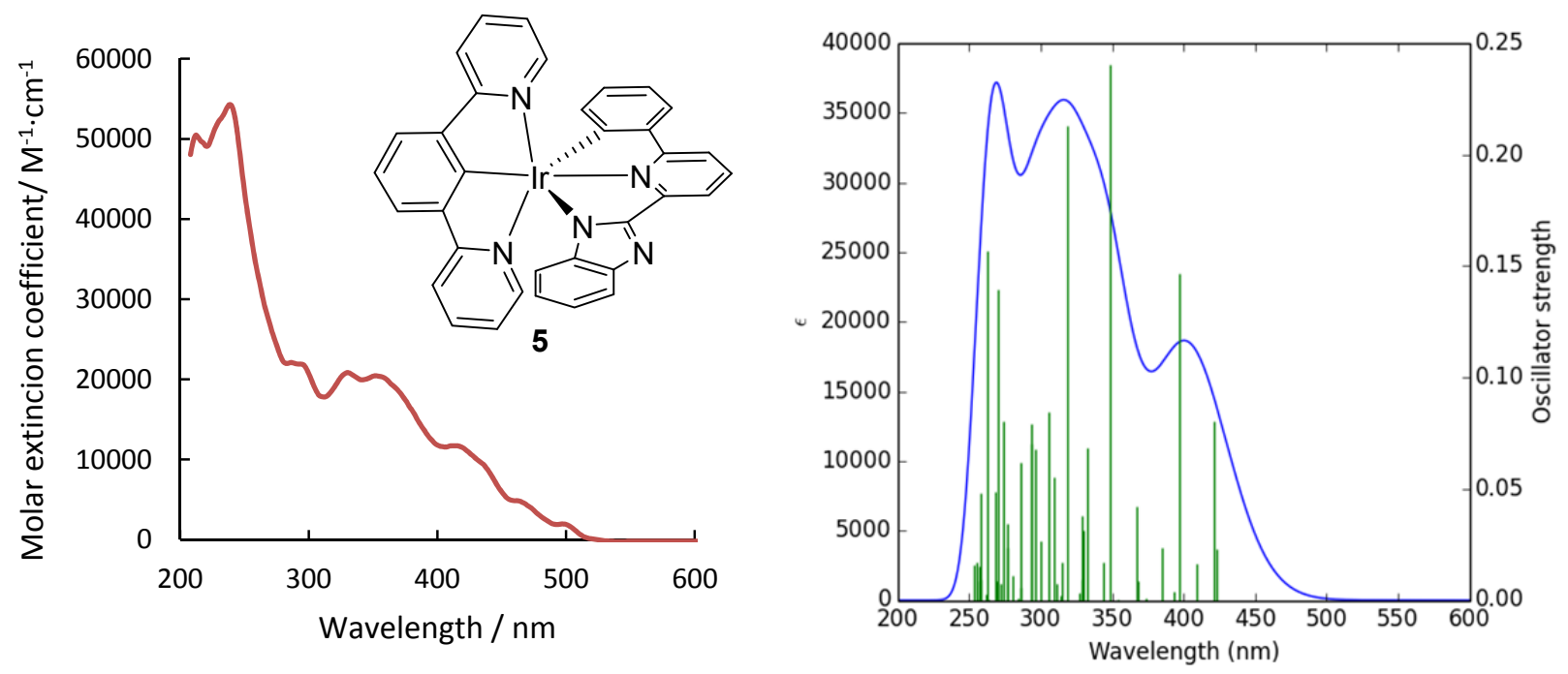

Figure S2. Observed UV-vis spectrum of complex 5 in 2-MeTHF $\left(2.0 \times 10^{-5} \mathrm{M}\right)$ and calculated (B3LYP(GD3)//SDD(f)/6-31G**) in THF. 

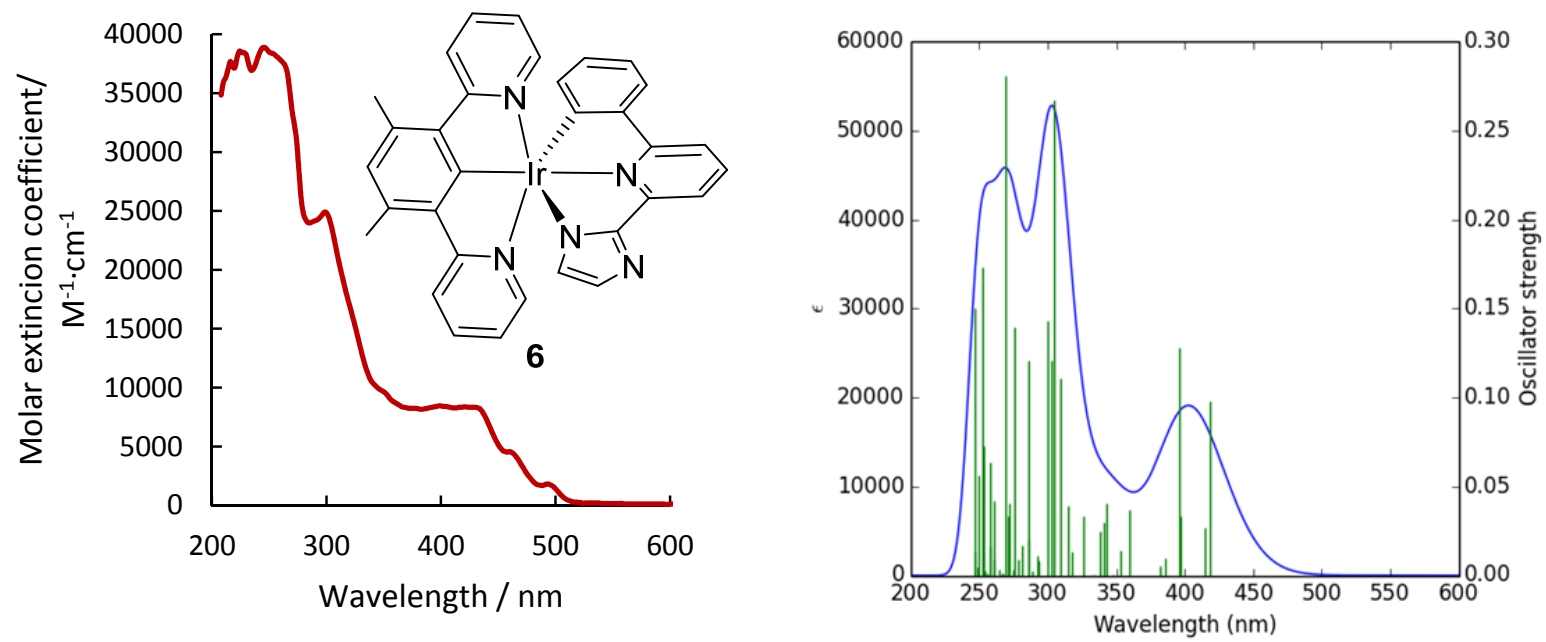

Figure S3. Observed UV-vis spectrum of complex 6 in 2-MeTHF $\left(2.5 \times 10^{-5} \mathrm{M}\right)$ and calculated (B3LYP(GD3)//SDD(f)/6-31G**) in THF.
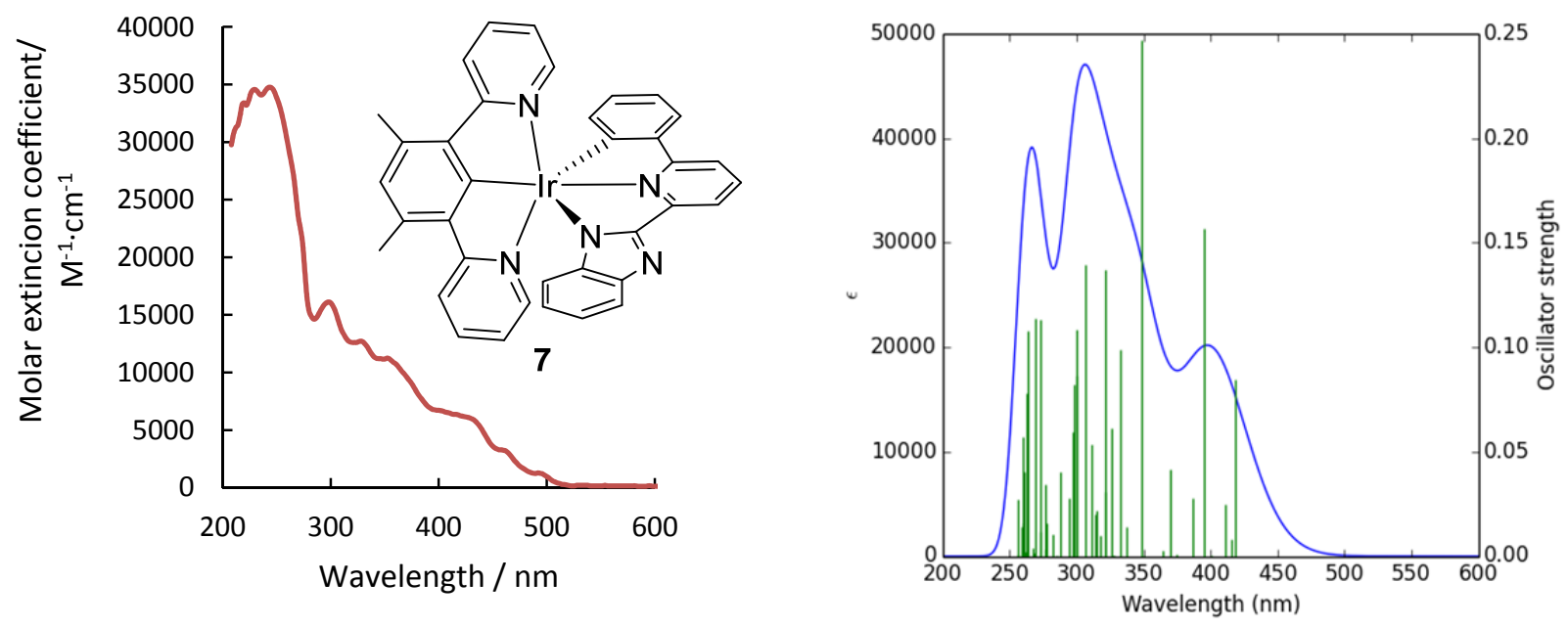

Figure S4. Observed UV-vis spectrum of complex 7 in 2-MeTHF $\left(2.5 \times 10^{-5} \mathrm{M}\right)$ and calculated (B3LYP(GD3)//SDD(f)/6-31G**) in THF.
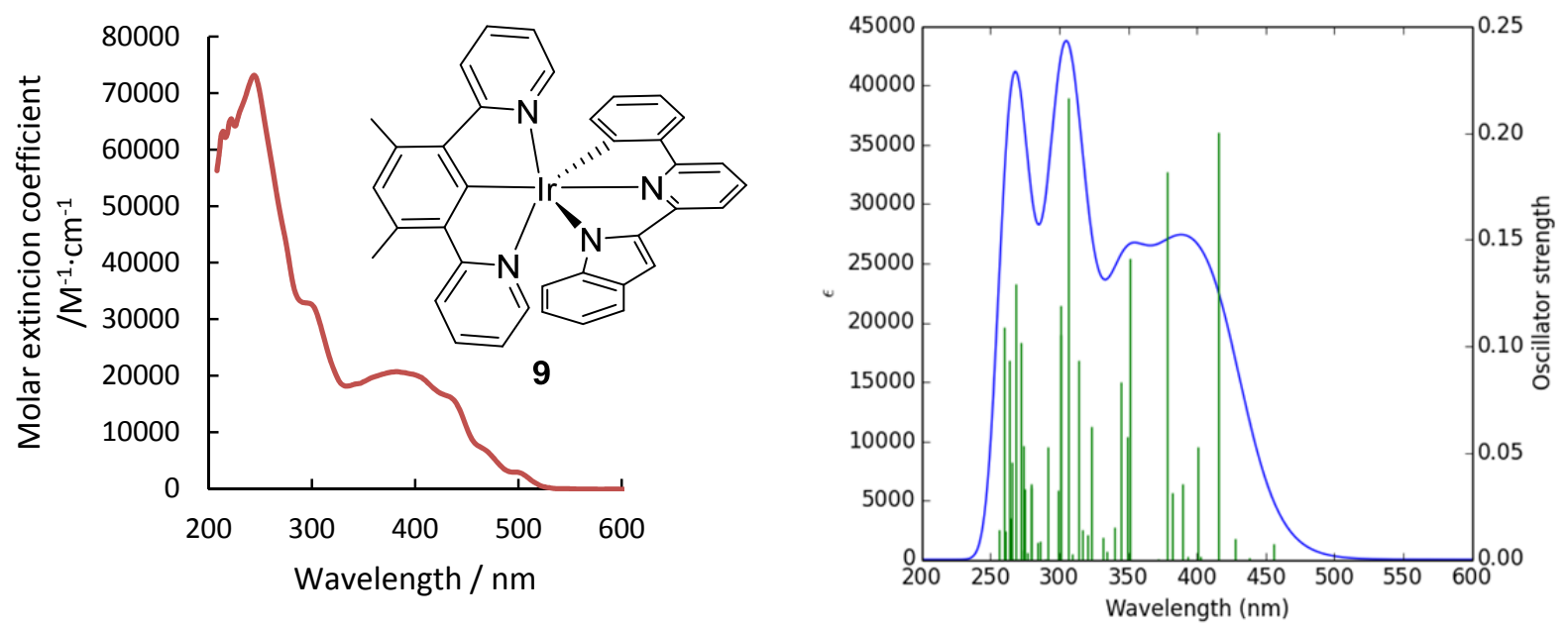

Figure S5. Observed UV-vis spectrum of complex 9 in 2-MeTHF $\left(2.5 \times 10^{-5} \mathrm{M}\right)$ and calculated (B3LYP(GD3)//SDD(f)/6-31G**) in THF. 


\section{Analysis of Computed UV/Vis Data for 4-7 and 9}

Selected transitions for the calculated UV spectra, energies, oscillator strengths, and molecular orbital contributions to the transitions are given in Tables S1, S3, S5, S7, and S9. In order to facilitate the understanding of the electronic transitions, an analysis of the change in charge density for each different group in which the molecules have been divided with the Gauss Sum program is included in Tables S2, S4, S6, S8, and S10.

Table S1: Selected transitions for the calculated UV spectrum of complex 4 in THF

\begin{tabular}{|c|c|c|c|c|c|}
\hline No. & $\lambda(\mathrm{nm})$ & $\begin{array}{l}\text { Osc. } \\
\text { Strength }\end{array}$ & Symmetry & Major contributions & Minor contributions \\
\hline 1 & 482 & 0 & Triplet & H-1->LUMO (96\%) & \\
\hline 7 & 424 & 0.0154 & Singlet & HOMO->LUMO (96\%) & \\
\hline 8 & 422 & 0.1015 & Singlet & $\begin{array}{l}\text { H-1->LUMO (82\%), HOMO- } \\
>\text { L+1 (15\%) }\end{array}$ & $H-2->L+1(2 \%)$ \\
\hline 10 & 398 & 0.1227 & Singlet & $\begin{array}{l}\text { H-1->LUMO (16\%), HOMO- } \\
>\text { L+1 (81\%) }\end{array}$ & \\
\hline 11 & 395 & 0.0412 & Singlet & HOMO->L+2 (90\%) & $\mathrm{H}-2->\mathrm{L}+2(3 \%), \mathrm{HOMO}->\mathrm{L}+3(4 \%)$ \\
\hline 41 & 307 & 0.1206 & Singlet & $H-3->L+2(70 \%)$ & $\begin{array}{l}\text { H-4->LUMO (2\%), H-3->L+3 (2\%), } \\
H-2->L+2(2 \%), H-2->L+4(9 \%), H- \\
1->L+5(6 \%), H O M O->L+3(3 \%)\end{array}$ \\
\hline 43 & 303 & 0.2367 & Singlet & $H-2->L+3(82 \%)$ & $H-1->L+5(6 \%)$, HOMO->L+3 (4\%) \\
\hline 68 & 276 & 0.1754 & Singlet & $\begin{array}{l}\text { H-6->LUMO (74\%), H-5- } \\
>\text { L+1 (12\%) }\end{array}$ & $H-3->L+5(2 \%), H-2->L+5(6 \%)$ \\
\hline 74 & 270 & 0.1073 & Singlet & $\begin{array}{c}\text { H-7->LUMO (16\%), H-5- } \\
>L+2(34 \%), H-4->L+3 \\
(10 \%), H-1->L+6(10 \%)\end{array}$ & $\begin{array}{l}H-5->L+3(2 \%), H-4->L+2(3 \%), H- \\
3->L+4(4 \%), H-1->L+8(7 \%), H-1- \\
>L+9(4 \%)\end{array}$ \\
\hline 82 & 263 & 0.0849 & Singlet & $\begin{array}{l}H-5->L+3(10 \%), H-4->L+3 \\
(63 \%)\end{array}$ & $\begin{array}{l}\text { H-7->LUMO (3\%), H-7->L+2 (6\%), } \\
H-5->L+2(9 \%), H-1->L+6(4 \%)\end{array}$ \\
\hline 98 & 248 & 0.0289 & Singlet & $\begin{array}{l}\text { H-9->LUMO (14\%), H-5- } \\
>\text { L+4 (70\%) }\end{array}$ & $\begin{array}{l}H-8->L+1(2 \%), H-7->L+2(3 \%), H- \\
4->L+4(5 \%)\end{array}$ \\
\hline
\end{tabular}

Table S2: Composition (\%) of the selected transitions for the calculated UV-vis spectrum of complex 4

\begin{tabular}{|c|c|c|c|c|c|c|}
\hline No. & $\lambda(\mathrm{nm})$ & $\begin{array}{c}\text { Osc. } \\
\text { Strength }\end{array}$ & Symmetry & Iridium & $\begin{array}{c}\text { dpyb } \\
\text { (5t-NCN) }\end{array}$ & $\begin{array}{c}\mathrm{NImpyC}_{6} \mathrm{H}_{4} \\
\left(4 \mathrm{t}^{\prime}-\mathrm{CNN}\right)\end{array}$ \\
\hline 1 & 482 & 0 & Triplet & $38-->2(-36)$ & 52-->97 (45) & $10-->1(-9)$ \\
\hline 7 & 424 & 0.0154 & Singlet & $16-->2(-14)$ & 4-->97 (93) & $80-->1(-79)$ \\
\hline 8 & 422 & 0.1015 & Singlet & $35-->3(-32)$ & $44-->96(52)$ & $21-->1(-20)$ \\
\hline 10 & 398 & 0.1227 & Singlet & $20-->6(-14)$ & $12-->92(80)$ & $68-->2(-66)$ \\
\hline 11 & 395 & 0.0412 & Singlet & $17-->4(-13)$ & $4-->2(-2)$ & 79-->94 (15) \\
\hline 41 & 307 & 0.1206 & Singlet & $52-->4(-48)$ & $16-->18(2)$ & $32-->78(46)$ \\
\hline 43 & 303 & 0.2367 & Singlet & $31-->3(-28)$ & $13-->15(2)$ & 57-->83 (26) \\
\hline 68 & 276 & 0.1754 & Singlet & $14-->2(-12)$ & $44-->96(52)$ & $43-->1(-42)$ \\
\hline 74 & 270 & 0.1073 & Singlet & $16-->18(2)$ & $34-->30(-4)$ & $50-->53(3)$ \\
\hline 82 & 263 & 0.0849 & Singlet & $4-->6(2)$ & $52-->10(-42)$ & $43-->84(41)$ \\
\hline 98 & 248 & 0.0289 & Singlet & $6-->2(-4)$ & $27-->87(60)$ & $67-->11(-56)$ \\
\hline
\end{tabular}


Table S3: Selected transitions for the calculated UV spectrum of complex 5 in THF

\begin{tabular}{|c|c|c|c|c|c|}
\hline No. & $\lambda(\mathrm{nm})$ & $\begin{array}{l}\text { Osc. } \\
\text { Strength }\end{array}$ & Symmetry & Major contributions & Minor contributions \\
\hline 1 & 481 & 0 & Triplet & H-1->LUMO (95\%) & \\
\hline 8 & 424 & 0.023 & Singlet & HOMO->LUMO (84\%) & $\begin{array}{l}\text { H-1->LUMO (8\%), HOMO- } \\
>\text { L+2 (3\%) }\end{array}$ \\
\hline 9 & 421 & 0.0801 & Singlet & $\begin{array}{l}\text { H-1->LUMO (72\%), HOMO- } \\
>\text { L+2 (16\%),HOMO->LUMO } \\
(10 \%)\end{array}$ & $H-2->L+1(2 \%)$ \\
\hline 13 & 397 & 0.1464 & Singlet & $\begin{array}{l}\text { H-1->LUMO (18\%), HOMO- } \\
>\text { L+2 (78\%) }\end{array}$ & \\
\hline 27 & 348 & 0.2403 & Singlet & $H-2->L+1(86 \%)$ & $\begin{array}{l}\mathrm{H}-3->\mathrm{LUMO}(4 \%), \mathrm{H}-2- \\
>\mathrm{L}+3(2 \%)\end{array}$ \\
\hline 43 & 319 & 0.2123 & Singlet & $H-3->L+1(88 \%)$ & $\mathrm{H}-2->\mathrm{L}+3(3 \%)$ \\
\hline 53 & 305 & 0.0846 & Singlet & $\begin{array}{l}H-4->L+1(68 \%), H-1->L+5 \\
(12 \%)\end{array}$ & $\begin{array}{l}H-2->L+3(3 \%), H-2->L+4 \\
(3 \%)\end{array}$ \\
\hline 60 & 294 & 0.0702 & Singlet & $H-5->L+2(92 \%)$ & \\
\hline 61 & 293 & 0.0793 & Singlet & $\begin{array}{l}\text { H-6->LUMO (12\%), H-5- } \\
>\text { LUMO (78\%) }\end{array}$ & $H-1->L+5(2 \%)$ \\
\hline 86 & 271 & 0.1394 & Singlet & $\begin{array}{l}H-6->L+1(59 \%), H-5->L+3 \\
(22 \%)\end{array}$ & $\begin{array}{l}H-4->L+4(2 \%), H-3->L+4 \\
(5 \%)\end{array}$ \\
\hline 92 & 263 & 0.1567 & Singlet & $H-6->L+3(68 \%)$ & $\begin{array}{l}\mathrm{H}-8->\mathrm{LUMO}(7 \%), \mathrm{H}-7- \\
>\mathrm{L}+2(5 \%), \mathrm{HOMO}->\mathrm{L}+7 \\
(7 \%)\end{array}$ \\
\hline
\end{tabular}

Table S4: Composition (\%) of the selected transitions for the calculated UV-vis spectrum of complex 5

\begin{tabular}{|c|c|c|c|c|c|c|}
\hline No. & $\lambda(\mathrm{nm})$ & $\begin{array}{c}\text { Osc. } \\
\text { Strength }\end{array}$ & Symmetry & Iridium & $\begin{array}{c}\text { dpyb } \\
\text { (5t-NCN) }\end{array}$ & $\begin{array}{c}\mathrm{NBzimpyC}_{6} \mathrm{H}_{4} \\
\left(4 \mathrm{t}^{\prime}-\mathrm{CNN}\right)\end{array}$ \\
\hline 1 & 481 & 0 & Triplet & $38-->2(-36)$ & 52-->97 (45) & $10-->1(-9)$ \\
\hline 8 & 424 & 0.023 & Singlet & $28-->2(-26)$ & 10-->97 (87) & $62-->1(-61)$ \\
\hline 9 & 421 & 0.0801 & Singlet & $35-->2(-33)$ & 40-->96 (56) & $25-->2(-23)$ \\
\hline 13 & 397 & 0.1464 & Singlet & $29-->6(-23)$ & 15-->92 (77) & $56-->2(-54)$ \\
\hline 27 & 348 & 0.2403 & Singlet & 7-->4 (-3) & $2-->6(4)$ & $91-->91(0)$ \\
\hline 43 & 319 & 0.2123 & Singlet & 21-->4 (-17) & $12-->2(-10)$ & $67-->95(28)$ \\
\hline 53 & 305 & 0.0846 & Singlet & $41-->4(-37)$ & $14-->18(4)$ & $44-->78(34)$ \\
\hline 60 & 294 & 0.0702 & Singlet & 1-->7 (6) & 79-->91 (12) & $20-->2(-18)$ \\
\hline 61 & 293 & 0.0793 & Singlet & $3-->2(-1)$ & 69-->97 (28) & $27-->1(-26)$ \\
\hline 86 & 271 & 0.1394 & Singlet & $13-->3(-10)$ & $29-->9(-20)$ & 59-->88 (29) \\
\hline 92 & 263 & 0.1567 & Singlet & $17-->2(-15)$ & $15-->14(-1)$ & $68-->84(16)$ \\
\hline
\end{tabular}


Table S5: Selected transitions for the calculated UV spectrum of complex 6 in THF

\begin{tabular}{|c|c|c|c|c|c|}
\hline No. & $\lambda(\mathrm{nm})$ & $\begin{array}{l}\text { Osc. } \\
\text { Strength }\end{array}$ & Symmetry & Major contributions & Minor contributions \\
\hline 1 & 473 & 0 & Triplet & H-1->LUMO (95\%) & \\
\hline 7 & 418 & 0.0977 & Singlet & $\begin{array}{l}\text { H-1->LUMO (79\%), HOMO- } \\
>\text { L+1 (17\%) }\end{array}$ & $H-2->L+1(2 \%)$ \\
\hline 8 & 414 & 0.0267 & Singlet & HOMO->LUMO (91\%) & $\begin{array}{l}\mathrm{H}-1->\mathrm{L}+1(3 \%), \text { HOMO->L+2 } \\
(2 \%)\end{array}$ \\
\hline 10 & 397 & 0.0332 & Singlet & HOMO->L+2 (87\%) & $\begin{array}{l}H-2->L+2(2 \%), H-1->L+1 \\
(4 \%), \text { HOMO->L+3 (3\%) }\end{array}$ \\
\hline 11 & 396 & 0.1276 & Singlet & $\begin{array}{l}\text { H-1->LUMO (19\%), HOMO- } \\
>\text { L+1 (77\%) }\end{array}$ & \\
\hline 38 & 318 & 0,0134 & Singlet & HOMO->L+5 (89\%) & $H-4->L+1(5 \%)$ \\
\hline 43 & 305 & 0.2672 & Singlet & $H-2->L+3(84 \%)$ & $\begin{array}{l}\mathrm{H}-1->\mathrm{L}+5(5 \%), \mathrm{HOMO}->\mathrm{L}+3 \\
(4 \%)\end{array}$ \\
\hline 47 & 300 & 0.1431 & Singlet & H-4->LUMO (73\%) & $\begin{array}{l}\text { H-3->LUMO (3\%), H-3->L+2 } \\
(3 \%), H-3->L+3(5 \%), H-2- \\
>L+4(6 \%), H-1->L+5(3 \%) \\
\text { HOMO->L+4 (2\%) }\end{array}$ \\
\hline 68 & 276 & 0.1397 & Singlet & H-6->LUMO (79\%) & \\
\hline 76 & 269 & 0.2803 & Singlet & $\begin{array}{l}H-5->L+2(52 \%), H-4->L+3 \\
(25 \%)\end{array}$ & $\begin{array}{l}H-7->L+2(2 \%), H-3->L+3 \\
(8 \%), H-3->L+4(2 \%)\end{array}$ \\
\hline 96 & 253 & 0.1734 & Singlet & $\begin{array}{l}H-7->L+1(20 \%), H-4->L+5 \\
(59 \%)\end{array}$ & $\begin{array}{l}\text { H-10->LUMO (7\%), H-9->L+1 } \\
(2 \%), H-8->\text { LUMO (5\%) }\end{array}$ \\
\hline
\end{tabular}

Table S6: Composition (\%) of the selected transitions for the calculated UV-vis spectrum of complex 6 in THF

\begin{tabular}{|c|c|c|c|c|c|c|}
\hline No. & $\lambda(\mathrm{nm})$ & $\begin{array}{c}\text { Osc. } \\
\text { Strength }\end{array}$ & Symmetry & Iridium & $\begin{array}{l}\text { dpyMeb } \\
\text { (5t-NCN) }\end{array}$ & $\begin{array}{c}\mathrm{NImpyC}_{6} \mathrm{H}_{4} \\
\left(4 \mathrm{t}^{\prime}-\mathrm{CNN}\right)\end{array}$ \\
\hline 1 & 473 & 0 & Triplet & $36-->2(-34)$ & 54-->96 (42) & $9-->2(-7)$ \\
\hline 7 & 418 & 0.0977 & Singlet & $33-->3(-30)$ & $45-->95(50)$ & $22-->2(-20)$ \\
\hline 8 & 414 & 0.0267 & Singlet & $19-->2(-17)$ & 7-->93 (86) & 74-->4 (-70) \\
\hline 10 & 397 & 0.0332 & Singlet & $19-->4(-15)$ & $8-->7(-1)$ & 73-->89 (16) \\
\hline 11 & 396 & 0.1276 & Singlet & $22-->6(-16)$ & 15-->92 (77) & $64-->2(-62)$ \\
\hline 38 & 318 & 0,0134 & Singlet & $17-->4(-13)$ & 9-->96 (87) & 73-->0 (-73) \\
\hline 43 & 305 & 0.2672 & Singlet & $27-->2(-25)$ & $17-->10(-7)$ & $55-->87(32)$ \\
\hline 47 & 300 & 0.1431 & Singlet & $13-->2(-11)$ & $64-->88(24)$ & $23-->10(-13)$ \\
\hline 68 & 276 & 0.1397 & Singlet & $18-->2(-16)$ & 46-->96 (50) & $36-->2(-34)$ \\
\hline 76 & 269 & 0.2803 & Singlet & $11-->3(-8)$ & $27-->6(-21)$ & 61-->91 (30) \\
\hline 96 & 253 & 0.1734 & Singlet & $7-->4(-3)$ & 55-->95 (40) & $38-->1(-37)$ \\
\hline
\end{tabular}


Table S7: Selected transitions for the calculated UV spectrum of complex 7 in THF

\begin{tabular}{|c|c|c|c|c|c|}
\hline No. & $\lambda(\mathrm{nm})$ & $\begin{array}{l}\text { Osc. } \\
\text { Strength }\end{array}$ & Symmetry & Major contributions & Minor contributions \\
\hline 1 & 472 & 0 & Triplet & H-1->LUMO (95\%) & \\
\hline 8 & 418 & 0.0848 & Singlet & $\begin{array}{l}\text { H-1->LUMO (76\%), HOMO- } \\
>\text { L+2 (22\%) }\end{array}$ & \\
\hline 9 & 416 & 0.008 & Singlet & $\begin{array}{l}\text { HOMO->LUMO (50\%), HOMO- } \\
>\text { L+1 (44\%) }\end{array}$ & $H-1->L+2(3 \%)$ \\
\hline 10 & 411 & 0.0249 & Singlet & $\begin{array}{l}\text { HOMO->LUMO (43\%), HOMO- } \\
>\text { L+1 (50\%) }\end{array}$ & $\begin{array}{l}\mathrm{H}-1->\mathrm{L}+2(3 \%), \mathrm{HOMO}->\mathrm{L}+3 \\
(2 \%)\end{array}$ \\
\hline 14 & 395 & 0.1568 & Singlet & $\begin{array}{l}\text { H-1->LUMO (22\%), HOMO- } \\
>\text { L+2 (73\%) }\end{array}$ & \\
\hline 27 & 349 & 0.2468 & Singlet & $\mathrm{H}-2->\mathrm{L}+1(89 \%)$ & $H-2->L+3(3 \%)$ \\
\hline 34 & 333 & 0.0987 & Singlet & $H-2->L+3(70 \%)$ & $\begin{array}{l}\text { H-4->LUMO (3\%), H-4->L+1 } \\
(5 \%), H-4->L+3(2 \%), H-3- \\
>\text { LUMO }(6 \%), H-3->L+1(4 \%), \\
\text { H-2->L+1 (3\%) }\end{array}$ \\
\hline 41 & 322 & 0.137 & Singlet & $H-3->L+1(63 \%)$ & $\begin{array}{l}\text { H-5->LUMO (8\%), H-4- } \\
\text { >LUMO (8\%), H-3->LUMO } \\
(3 \%), \text { HOMO->L+4 (6\%) }\end{array}$ \\
\hline 51 & 307 & 0.1393 & Singlet & $\begin{array}{l}H-5->L+1(19 \%), H-4->L+1 \\
(55 \%)\end{array}$ & $\begin{array}{l}H-2->L+3(4 \%), H-1->L+5 \\
(6 \%), H O M O->L+3(2 \%)\end{array}$ \\
\hline 54 & 300 & 0.1085 & Singlet & $\begin{array}{l}H-5->L+2(82 \%), H-4->L+2 \\
(10 \%)\end{array}$ & HOMO->L+5 (4\%) \\
\hline 83 & 273 & 0.1135 & Singlet & $H-6->L+1(75 \%)$ & $\begin{array}{l}H-6->\text { LUMO (3\%), H-6->L+3 } \\
(2 \%), H-5->L+3(7 \%), H-3- \\
>L+4(6 \%)\end{array}$ \\
\hline 98 & 259 & 0.0142 & Singlet & $\begin{array}{l}\text { H-5->L+4 (29\%), HOMO->L+7 } \\
(49 \%)\end{array}$ & $\begin{array}{l}H-8->L+1(4 \%), H-4->L+4 \\
(4 \%), H-1->L+9(2 \%)\end{array}$ \\
\hline
\end{tabular}

Table S8: Composition (\%) of the selected transitions for the calculated UV-vis spectrum of complex 7

\begin{tabular}{|c|c|c|c|c|c|c|}
\hline No. & $\lambda(\mathrm{nm})$ & $\begin{array}{c}\text { Osc. } \\
\text { Strength }\end{array}$ & Symmetry & Iridium & $\begin{array}{l}\text { dpyMeb } \\
\text { (5t-NCN) }\end{array}$ & $\begin{array}{c}\mathrm{NBzimpyC}_{6} \mathrm{H}_{4} \\
\left(4 \mathrm{t}^{\prime}-\mathrm{CNN}\right)\end{array}$ \\
\hline 1 & 472 & 0 & Triplet & $36-->2(-34)$ & 54-->96 (42) & $10-->2(-8)$ \\
\hline 8 & 418 & 0.0848 & Singlet & $34-->3(-31)$ & 44-->95 (51) & $22-->2(-20)$ \\
\hline 9 & 416 & 0.008 & Singlet & $29-->3(-26)$ & $9-->53(44)$ & $62-->44(-18)$ \\
\hline 10 & 411 & 0.0249 & Singlet & $29-->3(-26)$ & $9-->45(36)$ & $62-->52(-10)$ \\
\hline 14 & 395 & 0.1568 & Singlet & $30-->6(-24)$ & 19-->92 (73) & $51->2(-49)$ \\
\hline 27 & 349 & 0.2468 & Singlet & $7-->4(-3)$ & $1-->2(1)$ & $92-->94(2)$ \\
\hline 34 & 333 & 0.0987 & Singlet & $11-->2(-9)$ & $8-->10(2)$ & $81-->88(7)$ \\
\hline 41 & 322 & 0.137 & Singlet & $19-->3(-16)$ & $28-->29(1)$ & $53-->67(14)$ \\
\hline 51 & 307 & 0.1393 & Singlet & $30-->4(-26)$ & $38-->9(-29)$ & $32-->88(56)$ \\
\hline 54 & 300 & 0.1085 & Singlet & $17-->7(-10)$ & $36-->91(55)$ & $46-->2(-44)$ \\
\hline 83 & 273 & 0.1135 & Singlet & $15-->3(-12)$ & $12-->11(-1)$ & $73-->86(13)$ \\
\hline 98 & 259 & 0.0142 & Singlet & $24-->3(-21)$ & 21-->37 (16) & $55-->60(5)$ \\
\hline
\end{tabular}


Table S9: Selected transitions for the calculated UV spectrum of complex 9 in THF

\begin{tabular}{|c|c|c|c|c|c|}
\hline No. & $\lambda(\mathrm{nm})$ & $\begin{array}{l}\text { Osc. } \\
\text { Strength }\end{array}$ & Symmetry & Major contributions & Minor contributions \\
\hline 1 & 530 & 0 & Triplet & $\begin{array}{l}\text { HOMO->L+2 (39\%), } \\
\text { HOMO->L+3 (40\%) }\end{array}$ & $\begin{array}{l}H-2->L+2(3 \%), H-2->L+3 \\
(8 \%), H O M O->L+7(2 \%)\end{array}$ \\
\hline 6 & 456 & 0,0072 & Singlet & HOMO->LUMO (98\%) & \\
\hline 9 & 438 & 0,0008 & Singlet & $\begin{array}{l}\text { H-1->LUMO (17\%), } \\
\text { HOMO->L+1 (79\%) }\end{array}$ & \\
\hline 13 & 416 & 0,2004 & Singlet & $\begin{array}{l}\text { H-1->LUMO (79\%), } \\
\text { HOMO->L+1 (18\%) }\end{array}$ & \\
\hline 21 & 382 & 0,0315 & Singlet & $H-2->L+1(92 \%)$ & \\
\hline 22 & 379 & 0,1821 & Singlet & $\begin{array}{l}\mathrm{H}-2->\mathrm{L}+2(59 \%), \mathrm{H}-1- \\
>\mathrm{L}+1(18 \%)\end{array}$ & $\begin{array}{l}\text { H-2->LUMO (3\%), HOMO- } \\
>\text { L+2 (7\%), HOMO->L+3 (8\%) }\end{array}$ \\
\hline 30 & 352 & 0,1412 & Singlet & $\begin{array}{l}\mathrm{H}-2->\mathrm{L}+3(58 \%), \mathrm{HOMO}- \\
>\mathrm{L}+4(30 \%)\end{array}$ & H-3->LUMO (3\%) \\
\hline 34 & 344 & 0,0832 & Singlet & $\begin{array}{l}\text { H-4->LUMO (14\%), H-3- } \\
>\text { LUMO }(77 \%)\end{array}$ & $H-2->L+3(3 \%)$ \\
\hline 56 & 307 & 0,2163 & Singlet & $H-4->L+2(74 \%)$ & $\begin{array}{l}\mathrm{H}-5->\mathrm{LUMO}(3 \%), \mathrm{H}-5->\mathrm{L}+2 \\
(3 \%), \mathrm{H}-4->\mathrm{L}+3(7 \%), \mathrm{H}-2- \\
>\mathrm{L}+3(2 \%)\end{array}$ \\
\hline 60 & 301 & 0,1057 & Singlet & $H-5->L+1$ (89\%) & $H-2->L+5(6 \%)$ \\
\hline 89 & 272 & 0,1016 & Singlet & $\begin{array}{l}H-6->L+1(60 \%), H-3- \\
>L+5(15 \%)\end{array}$ & $\begin{array}{l}\text { H-7->LUMO (4\%), H-4->L+5 } \\
(7 \%), \text { HOMO->L+10 (3\%) }\end{array}$ \\
\hline 91 & 268 & 0,1293 & Singlet & $H-6->L+2(75 \%)$ & $\begin{array}{l}H-6->L+3(4 \%), H-5->L+3 \\
(4 \%), H O M O->L+7(4 \%)\end{array}$ \\
\hline
\end{tabular}

Table S10: Composition (\%) of the selected transitions for the calculated UV-vis spectrum of complex 9

\begin{tabular}{ccccccc}
\hline No. & $\lambda \mathbf{( n m})$ & $\begin{array}{c}\text { Osc. } \\
\text { Strength }\end{array}$ & Symmetry & Iridium & $\begin{array}{c}\text { dpyMeb } \\
\text { (5t-NCN) }\end{array}$ & $\begin{array}{c}\text { IndpyC } \mathbf{H}_{4} \\
\text { (4t'-CNN) }\end{array}$ \\
\hline $\mathbf{1}$ & 530 & 0 & Triplet & $16-->3(-13)$ & $4-->2(-2)$ & $80-->95(15)$ \\
\hline $\mathbf{6}$ & 456 & 0,0072 & Singlet & $17-->2(-15)$ & $4-->96(92)$ & $80-->2(-78)$ \\
$\mathbf{9}$ & 438 & 0,0008 & Singlet & $20-->6(-14)$ & $12-->92(80)$ & $67-->2(-65)$ \\
$\mathbf{1 3}$ & 416 & 0,2004 & Singlet & $33-->3(-30)$ & $44-->95(51)$ & $23-->2(-21)$ \\
$\mathbf{2 1}$ & 382 & 0,0315 & Singlet & $10-->7(-3)$ & $3-->91(88)$ & $87-->2(-85)$ \\
$\mathbf{2 2}$ & 379 & 0,1821 & Singlet & $16-->4(-12)$ & $13-->22(9)$ & $71-->74(3)$ \\
$\mathbf{3 0}$ & 352 & 0,1412 & Singlet & $13-->2(-11)$ & $4-->36(32)$ & $83->63(-20)$ \\
$\mathbf{3 4}$ & 344 & 0,0832 & Singlet & $25-->2(-23)$ & $22-->93(71)$ & $54-->5(-49)$ \\
$\mathbf{5 6}$ & 307 & 0,2163 & Singlet & $49-->4(-45)$ & $20-->5(-15)$ & $31-->91(60)$ \\
$\mathbf{6 0}$ & 301 & 0,1057 & Singlet & $3-->7(4)$ & $61-->91(30)$ & $35->>2(-33)$ \\
$\mathbf{8 9}$ & 272 & 0,1016 & Singlet & $18-->7(-11)$ & $14-->91(77)$ & $67-->2(-65)$ \\
$\mathbf{9 1}$ & 268 & 0,1293 & Singlet & $14-->4(-10)$ & $12-->3(-9)$ & $74-->94(20)$ \\
\hline
\end{tabular}



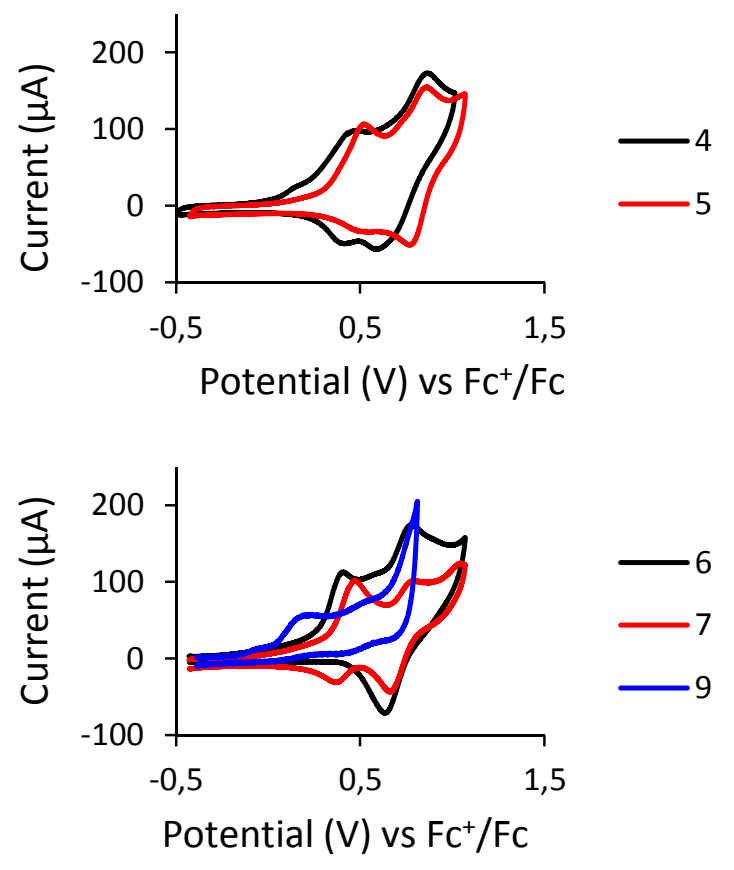

Figure S6. Cyclic voltammograms of complexes 4-7 and 9, in dichloromethane $\left(10^{-3} \mathrm{M}, 4-7\right)$ or acetonitrile $\left(10^{-3} \mathrm{M}, 9\right)$ solutions with $\mathrm{Bu}_{4} \mathrm{NPF}_{6}$ as supporting electrolyte $(0.1 \mathrm{M})$ at a scan rate of $100 \mathrm{mV} \mathrm{s}^{-1}$. The potentials were referenced to the ferrocene/ferrocenium $\left(\mathrm{Fc} / \mathrm{Fc}^{+}\right)$couple. 
Theoretical Analysis of Molecular Orbitals of Complexes 4-7 and 9

Molecular orbitals, energies, and population analysis (\%) are given in Tables S11 - S15 and Figures $\mathrm{S} 7-\mathrm{S} 12$.

Table S11: Composition of the frontier orbitals of complex 4

\begin{tabular}{|c|c|c|c|c|}
\hline Mo & $\mathrm{eV}$ & Iridium & $\begin{array}{l}\text { NCN-dpyb } \\
\text { (5t) }\end{array}$ & $\begin{array}{c}\text { CNN-NImpyC }{ }_{6} \mathrm{H}_{4} \\
\left(4 \mathrm{t}^{\prime}\right)\end{array}$ \\
\hline$L+10$ & 0,79 & 7 & 0 & 93 \\
\hline L+9 & 0,69 & 31 & 62 & 7 \\
\hline$L+8$ & 0,57 & 36 & 54 & 10 \\
\hline$L+7$ & 0,43 & 4 & 1 & 95 \\
\hline$L+6$ & 0,1 & 98 & 3 & 0 \\
\hline$L+5$ & $-0,54$ & 4 & 96 & 0 \\
\hline$L+4$ & $-0,75$ & 2 & 88 & 10 \\
\hline$L+3$ & $-0,94$ & 2 & 9 & 89 \\
\hline$L+2$ & $-1,2$ & 4 & 2 & 94 \\
\hline L+1 & $-1,33$ & 7 & 91 & 2 \\
\hline LUMO & $-1,59$ & 2 & 97 & 1 \\
\hline Hомо & $-5,08$ & 16 & 4 & 80 \\
\hline H-1 & $-5,23$ & 38 & 52 & 10 \\
\hline $\mathrm{H}-2$ & $-5,58$ & 31 & 10 & 59 \\
\hline $\mathrm{H}-3$ & $-5,92$ & 59 & 13 & 28 \\
\hline H-4 & $-6,2$ & 1 & 67 & 32 \\
\hline $\mathrm{H}-5$ & $-6,32$ & 4 & 25 & 70 \\
\hline H-6 & $-6,75$ & 12 & 50 & 37 \\
\hline $\mathrm{H}-7$ & $-6,82$ & 15 & 8 & 77 \\
\hline $\mathrm{H}-8$ & $-7,01$ & 10 & 13 & 77 \\
\hline H-9 & $-7,2$ & 14 & 27 & 59 \\
\hline H-10 & $-7,33$ & 11 & 50 & 39 \\
\hline
\end{tabular}




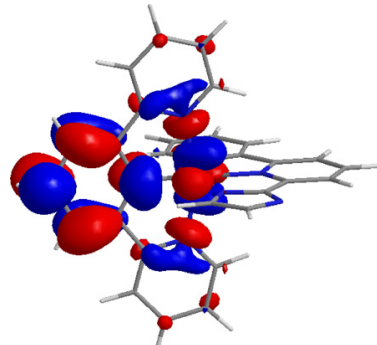

L+9

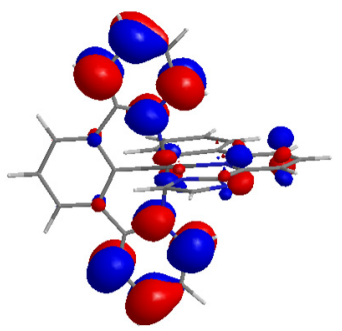

L+5

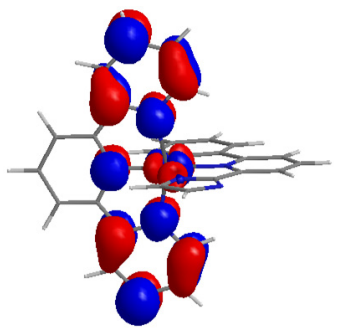

L+1

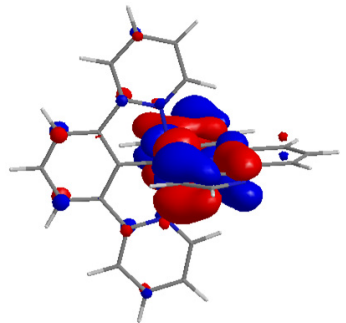

H-2

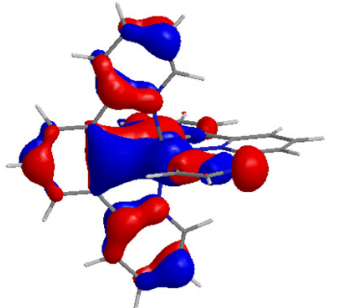

H-6

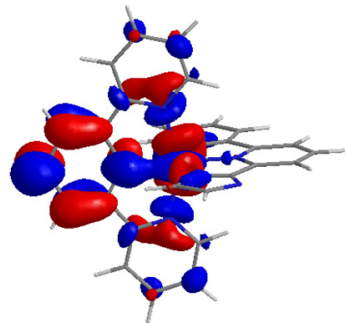

$L+8$

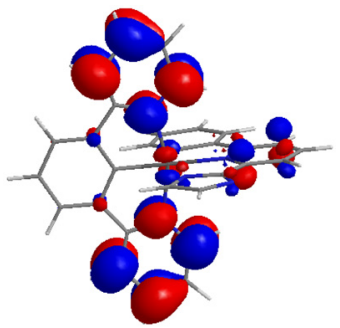

L+4

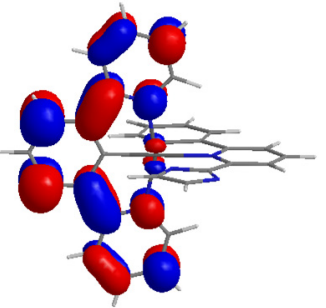

LUMO

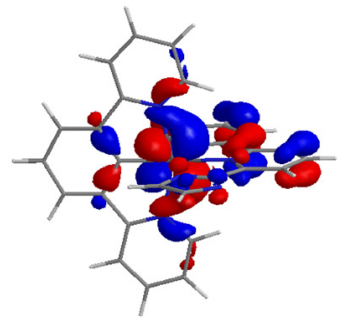

$\mathrm{H}-3$

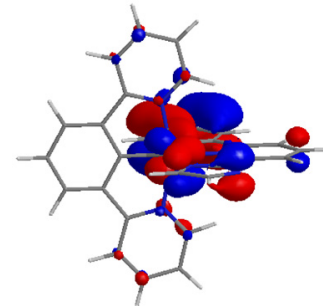

H-7

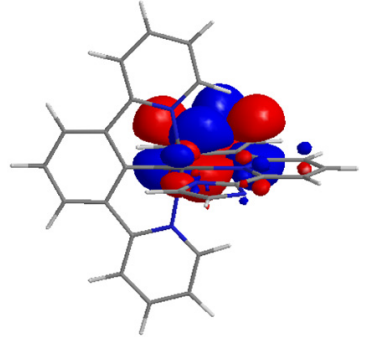

L+7

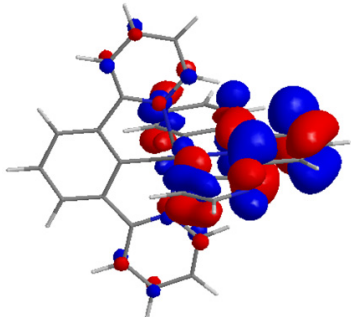

$L+3$

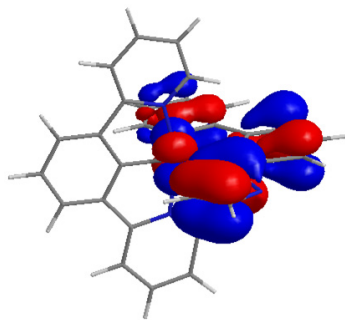

HOMO

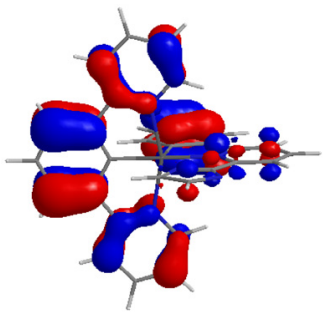

H-4

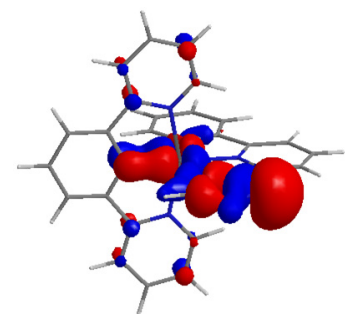

H-8

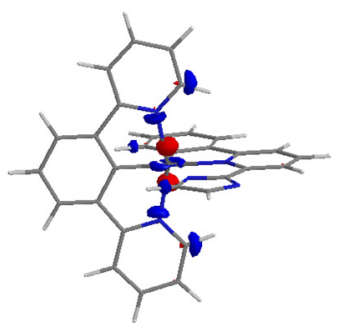

L+6

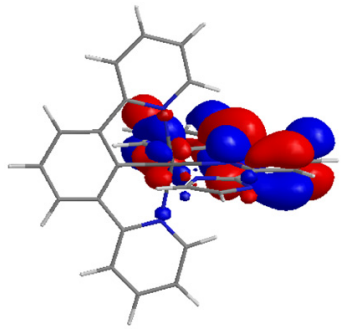

$\mathrm{L}+2$

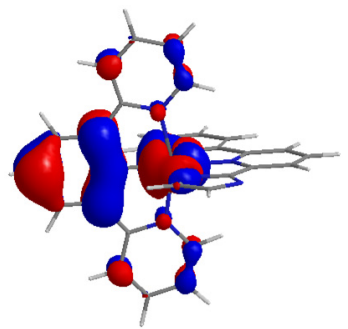

H-1

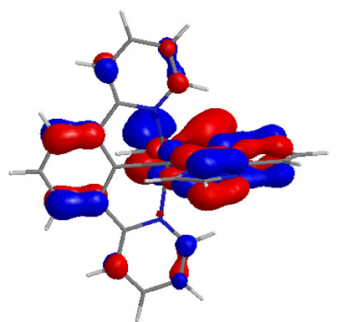

H-5

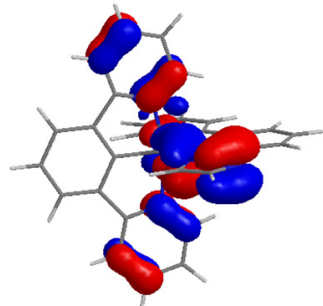

H-9

Figure S7. Frontier molecular orbitals of complex 4 (isovalue $0.03 \mathrm{au}$ ). 
Table S12: Composition of the frontier orbitals of complex 5

\begin{tabular}{|c|c|c|c|c|}
\hline MO & eV & Iridium & $\begin{array}{l}\text { NCN-dpyb } \\
(5 t)\end{array}$ & $\begin{array}{c}\text { CNN-NBzimpyC } \mathrm{C}_{6} \mathrm{H}_{4} \\
\left(4 \mathrm{t}^{\prime}\right)\end{array}$ \\
\hline$L+10$ & 0,69 & 37 & 54 & 8 \\
\hline L+9 & 0,58 & 30 & 62 & 7 \\
\hline$L+8$ & 0,48 & 3 & 0 & 97 \\
\hline L+7 & 0,27 & 2 & 1 & 97 \\
\hline$L+6$ & 0,1 & 97 & 4 & 0 \\
\hline$L+5$ & $-0,54$ & 4 & 96 & 0 \\
\hline$L+4$ & $-0,77$ & 2 & 95 & 3 \\
\hline$L+3$ & $-1,22$ & 2 & 2 & 97 \\
\hline$L+2$ & $-1,33$ & 7 & 91 & 2 \\
\hline$L+1$ & $-1,36$ & 4 & 2 & 95 \\
\hline LUMO & $-1,6$ & 2 & 97 & 1 \\
\hline HOMO & $-5,14$ & 27 & 6 & 67 \\
\hline H-1 & $-5,24$ & 38 & 52 & 10 \\
\hline $\mathrm{H}-2$ & $-5,46$ & 6 & 1 & 92 \\
\hline $\mathrm{H}-3$ & $-5,82$ & 22 & 13 & 66 \\
\hline $\mathrm{H}-4$ & $-6,05$ & 45 & 9 & 46 \\
\hline H-5 & $-6,21$ & 1 & 79 & 20 \\
\hline H-6 & $-6,48$ & 15 & 12 & 73 \\
\hline $\mathrm{H}-7$ & $-6,78$ & 15 & 56 & 29 \\
\hline $\mathrm{H}-8$ & $-6,94$ & 21 & 24 & 54 \\
\hline H-9 & $-7,1$ & 5 & 6 & 89 \\
\hline H-10 & $-7,39$ & 12 & 51 & 37 \\
\hline
\end{tabular}




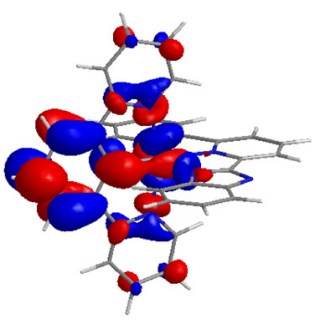

L+9

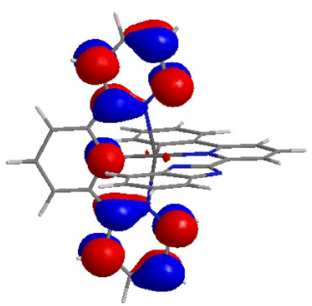

L+5

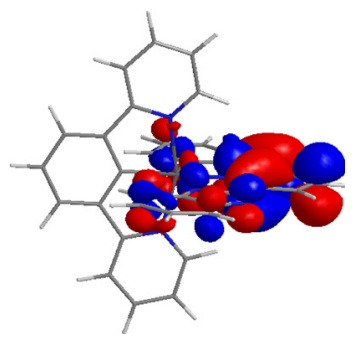

L+1

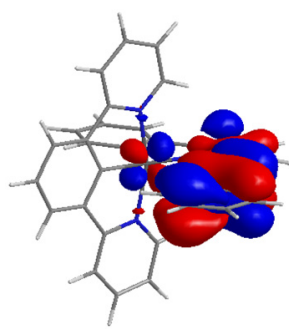

H-2

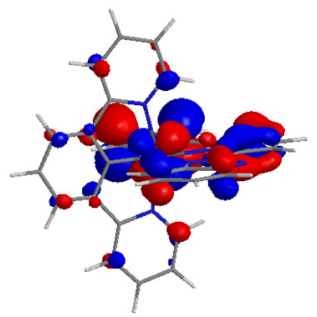

H-6

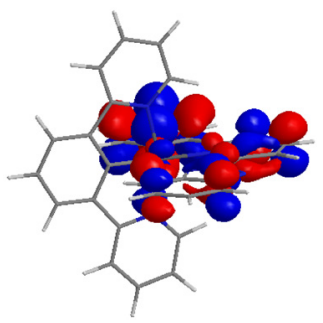

$L+8$

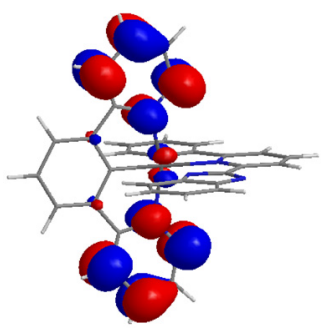

L+4

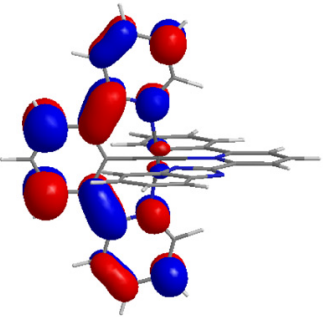

LUMO

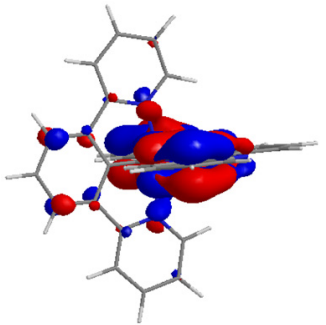

H-3

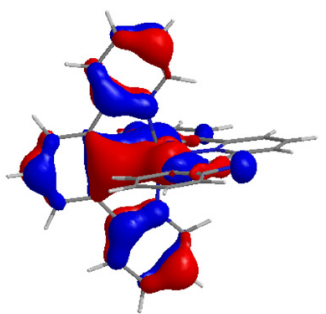

H-7

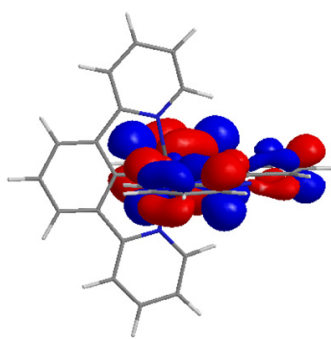

L+7

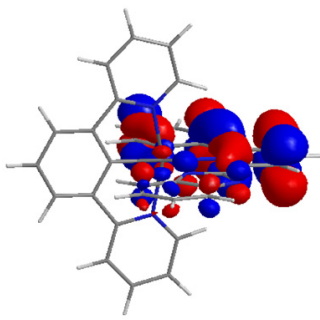

$L+3$

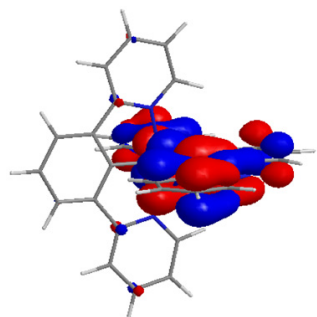

HOMO

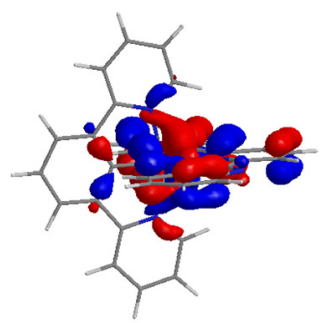

H-4

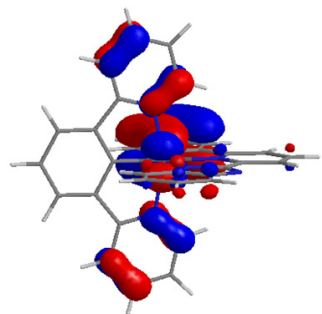

H-8

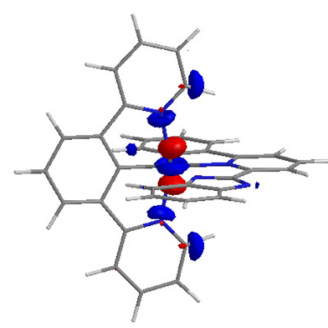

L+6

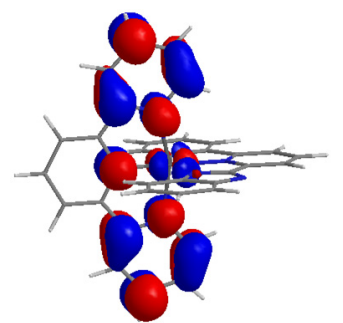

L+2

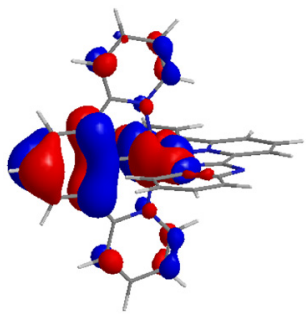

H-1

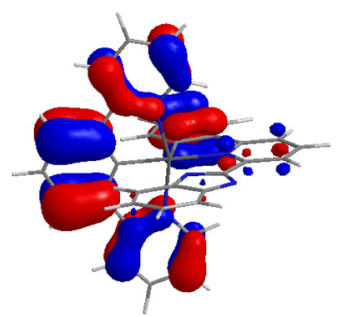

H-5

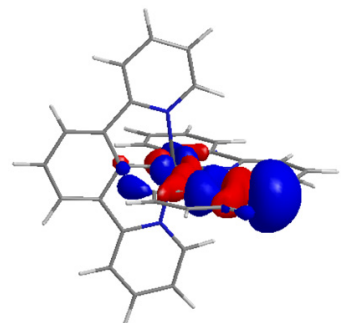

H-9

Figure S8. Frontier molecular orbitals of complex 5 (isovalue $0.03 \mathrm{au}$ ). 
Table S13: Composition of the frontier orbitals of complex 6

\begin{tabular}{|c|c|c|c|c|}
\hline MO & eV & Iridium & $\begin{array}{c}\text { NCN-dpyMeb } \\
(5 t)\end{array}$ & $\begin{array}{c}\mathrm{CNN}-\mathrm{N} I m p y \mathrm{C}_{6} \mathrm{H}_{(} \\
\left(4 \mathrm{t}^{\prime}\right)\end{array}$ \\
\hline$L+10$ & 0,84 & 32 & 62 & 6 \\
\hline$L+9$ & 0,79 & 7 & 0 & 93 \\
\hline$L+8$ & 0,7 & 38 & 51 & 11 \\
\hline$L+7$ & 0,45 & 4 & 1 & 95 \\
\hline$L+6$ & 0,1 & 98 & 2 & 0 \\
\hline$L+5$ & $-0,53$ & 4 & 96 & 0 \\
\hline$L+4$ & $-0,66$ & 2 & 91 & 8 \\
\hline$L+3$ & $-0,93$ & 2 & 6 & 92 \\
\hline$L+2$ & $-1,19$ & 4 & 3 & 93 \\
\hline$L+1$ & $-1,28$ & 7 & 91 & 2 \\
\hline LUMO & $-1,49$ & 2 & 96 & 2 \\
\hline номо & $-5,06$ & 18 & 5 & 77 \\
\hline H-1 & $-5,16$ & 36 & 54 & 9 \\
\hline H-2 & $-5,53$ & 27 & 16 & 57 \\
\hline $\mathrm{H}-3$ & $-5,89$ & 58 & 17 & 25 \\
\hline H-4 & $-6,03$ & 4 & 76 & 20 \\
\hline $\mathrm{H}-5$ & $-6,29$ & 5 & 7 & 88 \\
\hline H-6 & $-6,66$ & 16 & 52 & 31 \\
\hline H-7 & $-6,79$ & 15 & 9 & 76 \\
\hline $\mathrm{H}-8$ & $-6,99$ & 10 & 8 & 82 \\
\hline H-9 & $-7,15$ & 12 & 26 & 62 \\
\hline H-10 & $-7,24$ & 11 & 52 & 37 \\
\hline
\end{tabular}




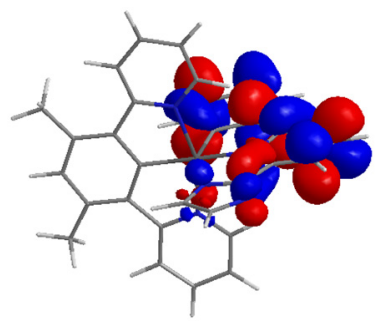

$L+9$

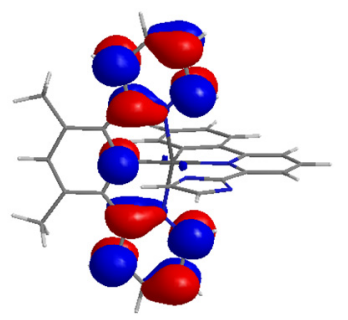

L+5

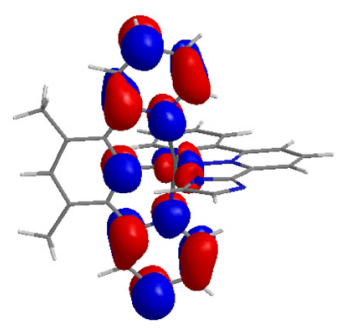

L+1

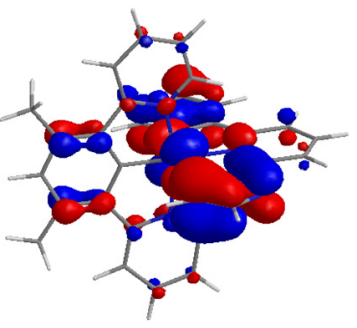

H-2

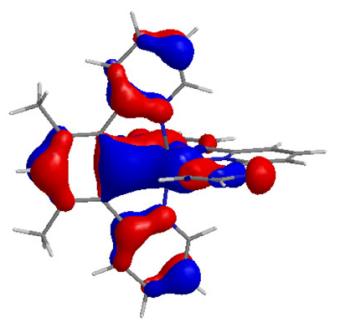

H-6

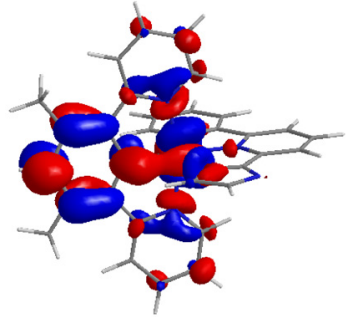

L+8

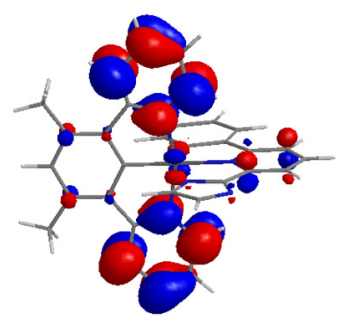

L+4

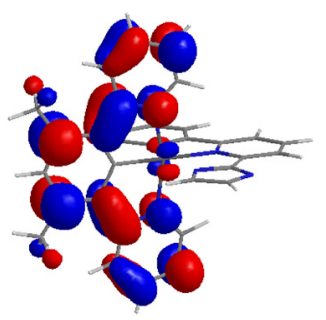

LUMO

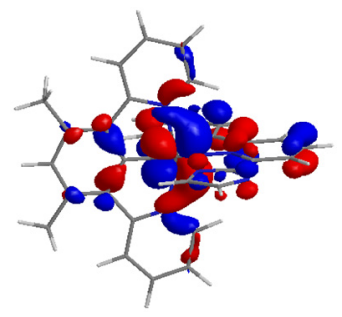

H-3

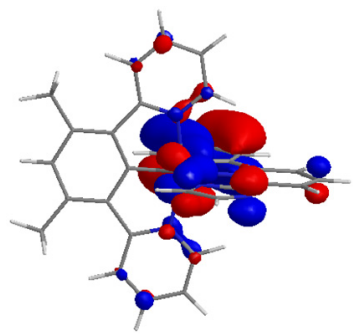

H-7

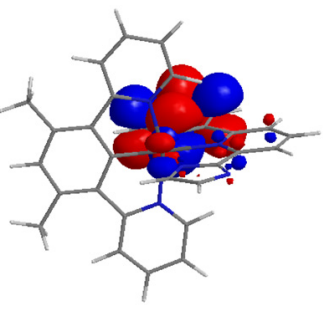

L+7

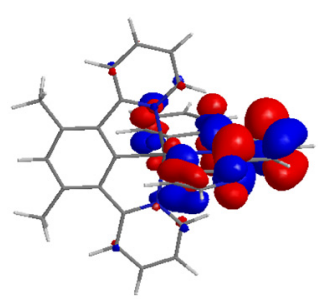

$L+3$

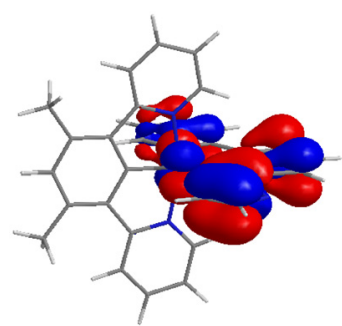

HOMO

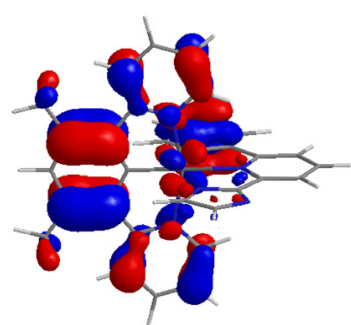

H-4

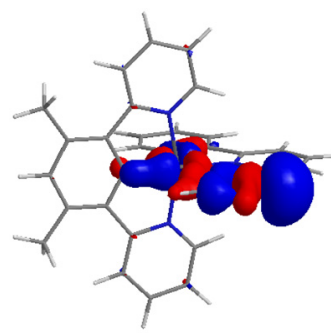

H-8

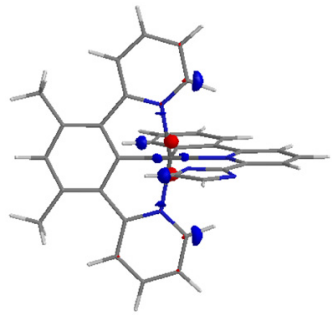

L+6

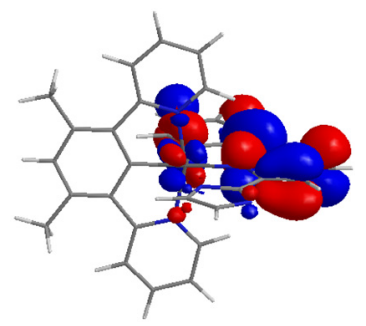

$\mathrm{L}+2$

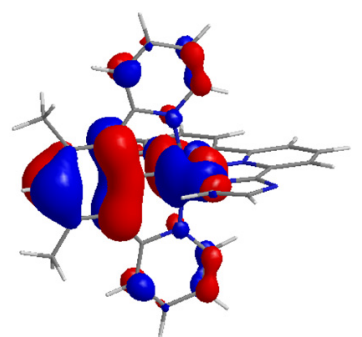

H-1

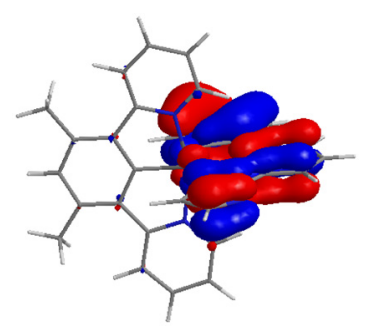

H-5

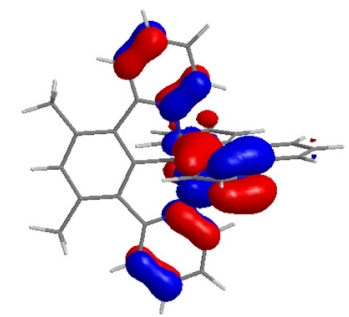

H-9

Figure S9. Frontier molecular orbitals of complex 6 (isovalue $0.03 \mathrm{au}$ ). 
Table S14: Composition of the frontier orbitals of complex 7

\begin{tabular}{|c|c|c|c|c|}
\hline MO & eV & Iridium & $\begin{array}{c}\text { NCN-dpyMeb } \\
\text { (5t-) }\end{array}$ & $\begin{array}{c}\text { CNN-NBzimpyC } \mathrm{C}_{6} \mathrm{H}_{4} \\
\left(4 \mathrm{t}^{\prime}\right)\end{array}$ \\
\hline$L+10$ & 0,84 & 40 & 52 & 8 \\
\hline$L+9$ & 0,71 & 31 & 61 & 8 \\
\hline$L+8$ & 0,49 & 3 & 1 & 96 \\
\hline$L+7$ & 0,28 & 2 & 1 & 97 \\
\hline$L+6$ & 0,11 & 97 & 3 & 0 \\
\hline$L+5$ & $-0,54$ & 4 & 96 & 0 \\
\hline$L+4$ & $-0,68$ & 2 & 95 & 3 \\
\hline$L+3$ & $-1,21$ & 2 & 2 & 96 \\
\hline$L+2$ & $-1,28$ & 7 & 91 & 2 \\
\hline L+1 & $-1,36$ & 4 & 2 & 94 \\
\hline LUMO & $-1,5$ & 2 & 96 & 2 \\
\hline HOMO & $-5,11$ & 29 & 8 & 63 \\
\hline H-1 & $-5,17$ & 36 & 54 & 10 \\
\hline H-2 & $-5,45$ & 7 & 1 & 92 \\
\hline H-3 & $-5,77$ & 16 & 27 & 57 \\
\hline H-4 & -6 & 36 & 40 & 24 \\
\hline $\mathrm{H}-5$ & $-6,06$ & 15 & 37 & 48 \\
\hline H-6 & $-6,45$ & 15 & 9 & 76 \\
\hline H-7 & $-6,68$ & 18 & 56 & 27 \\
\hline H-8 & $-6,89$ & 20 & 24 & 56 \\
\hline H-9 & $-7,08$ & 5 & 4 & 91 \\
\hline H-10 & $-7,3$ & 12 & 54 & 35 \\
\hline
\end{tabular}




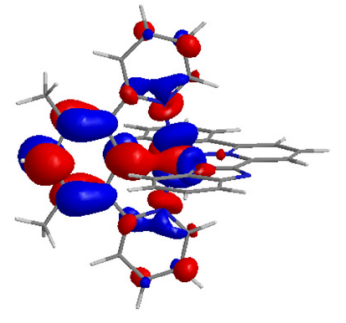

$L+9$

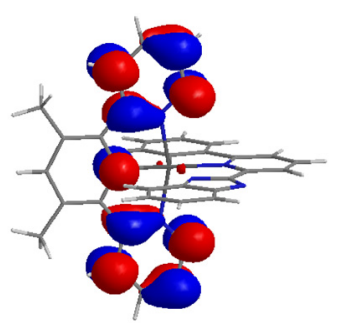

$L+5$

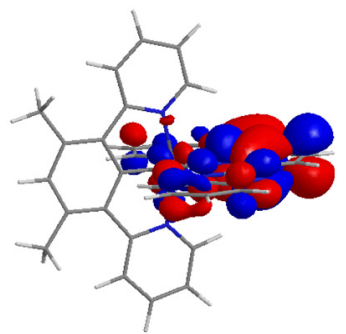

L+1

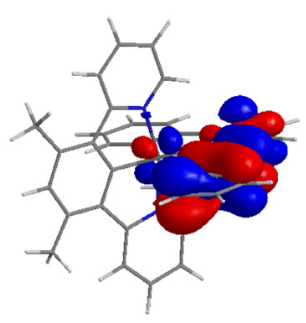

H-2

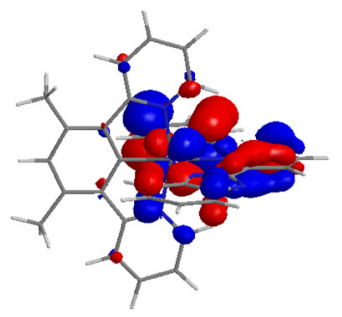

H-6

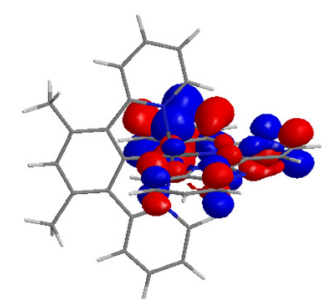

L+8

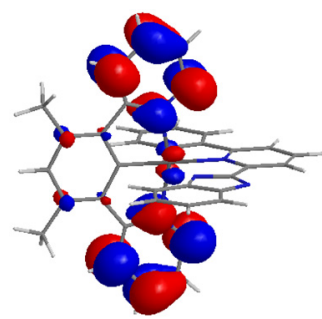

L+4

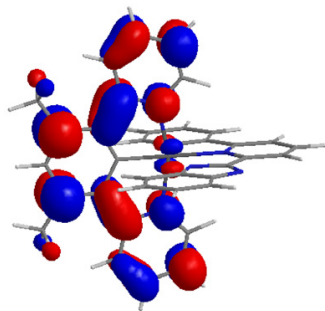

LUMO

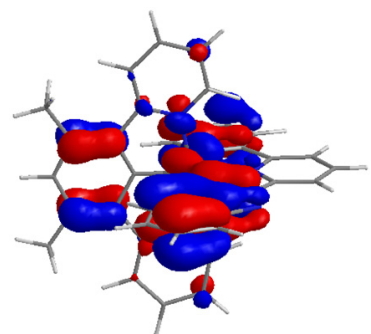

H-3

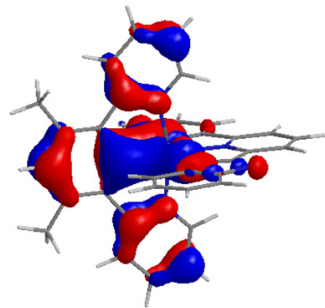

H-7

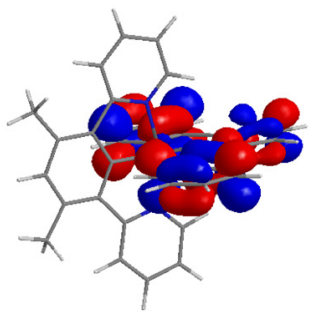

$L+7$

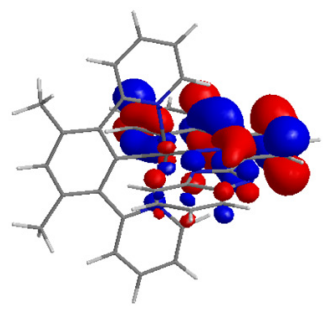

$L+3$

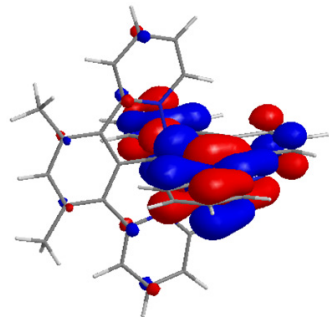

HOMO

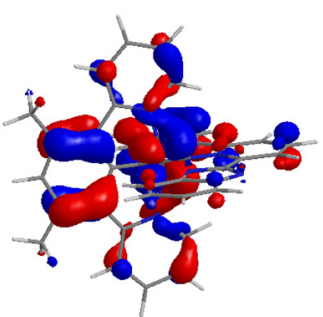

H-4

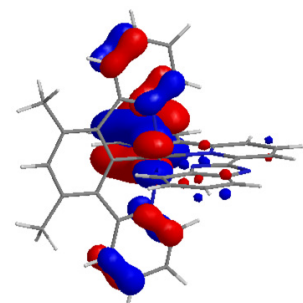

H-8

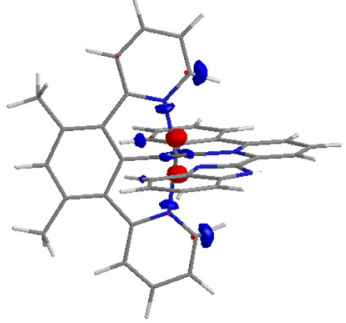

$L+6$

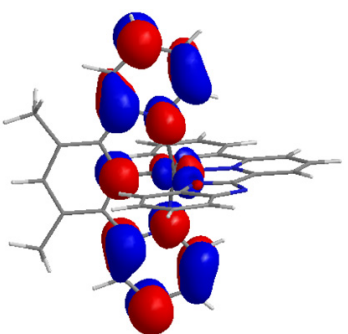

L+2

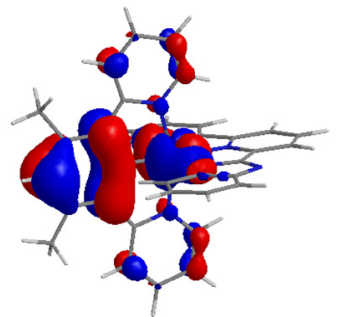

H-1

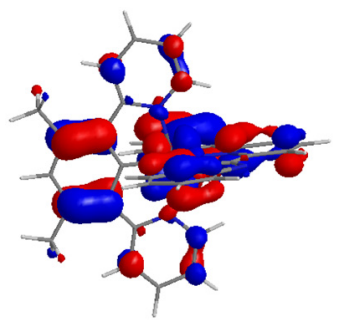

H-5

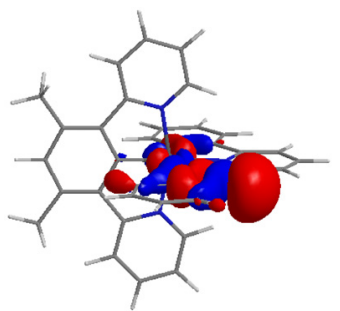

H-9

Figure S10. Frontier molecular orbitals of complex 7 (isovalue $0.03 \mathrm{au}$ ). 
Table S15: Composition of the frontier orbitals of complex 9

\begin{tabular}{|c|c|c|c|c|}
\hline MO & eV & Iridium & $\begin{array}{c}\text { NCN-dpyMeb } \\
\text { (5t) }\end{array}$ & $\begin{array}{c}\text { CNN-IndpyC } \mathrm{C}_{6} \mathrm{H} \\
\left(4 \mathrm{t}^{\prime}\right)\end{array}$ \\
\hline$L+10$ & 0,89 & 50 & 41 & 9 \\
\hline$L+9$ & 0,78 & 23 & 71 & 6 \\
\hline$L+8$ & 0,56 & 3 & 1 & 97 \\
\hline$L+7$ & 0,36 & 3 & 1 & 96 \\
\hline$L+6$ & 0,12 & 98 & 3 & 0 \\
\hline$L+5$ & $-0,5$ & 4 & 96 & 0 \\
\hline$L+4$ & $-0,64$ & 2 & 94 & 4 \\
\hline$L+3$ & $-1,11$ & 1 & 2 & 97 \\
\hline$L+2$ & $-1,22$ & 4 & 3 & 93 \\
\hline$L+1$ & $-1,23$ & 7 & 91 & 2 \\
\hline LUMO & $-1,46$ & 2 & 96 & 2 \\
\hline HOMO & $-4,76$ & 17 & 4 & 80 \\
\hline H-1 & $-5,09$ & 36 & 53 & 11 \\
\hline $\mathrm{H}-2$ & $-5,09$ & 10 & 3 & 87 \\
\hline H-3 & $-5,64$ & 20 & 23 & 57 \\
\hline $\mathrm{H}-4$ & $-5,93$ & 54 & 17 & 29 \\
\hline H-5 & $-5,99$ & 3 & 65 & 32 \\
\hline H-6 & $-6,38$ & 14 & 9 & 76 \\
\hline H-7 & $-6,6$ & 16 & 57 & 27 \\
\hline $\mathrm{H}-8$ & $-6,83$ & 19 & 21 & 60 \\
\hline H-9 & $-7,16$ & 4 & 7 & 89 \\
\hline H-10 & $-7,21$ & 11 & 53 & 36 \\
\hline
\end{tabular}




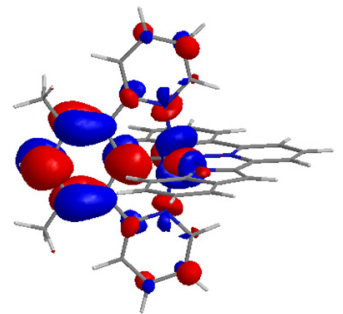

L+9

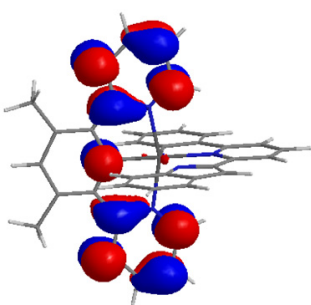

$L+5$

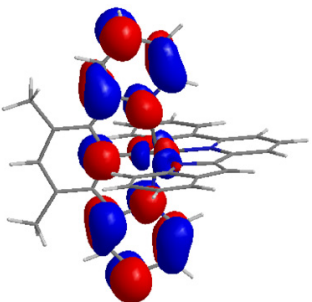

L+1

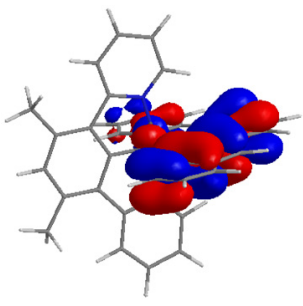

$\mathrm{H}-2$

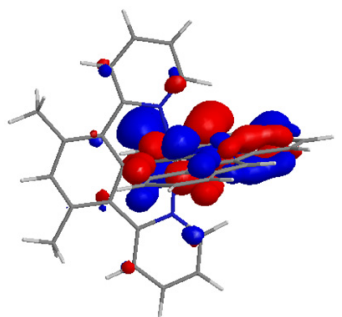

H-6

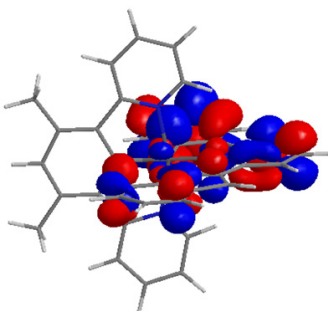

L+8

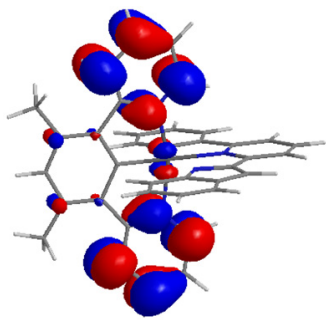

L+4

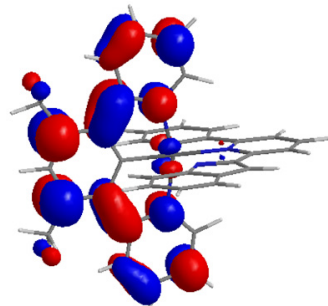

LUMO

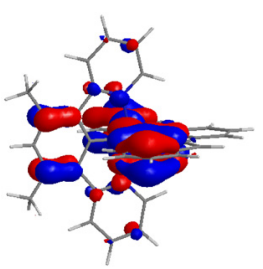

H-3

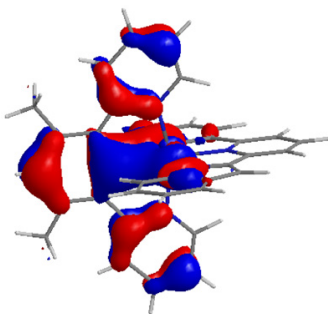

H-7

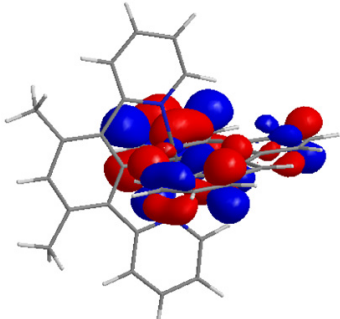

L+7

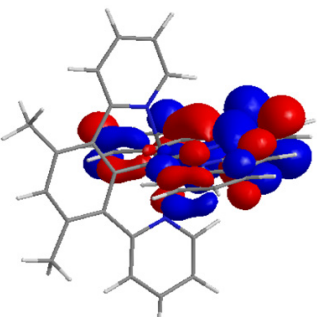

$L+3$

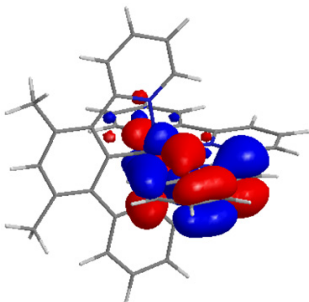

HOMO

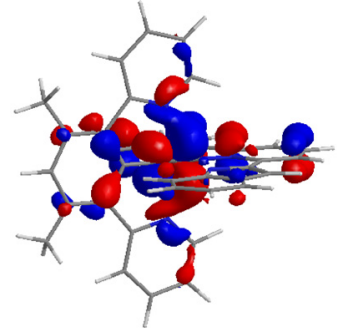

H-4

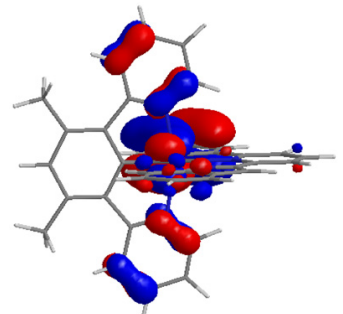

H-8
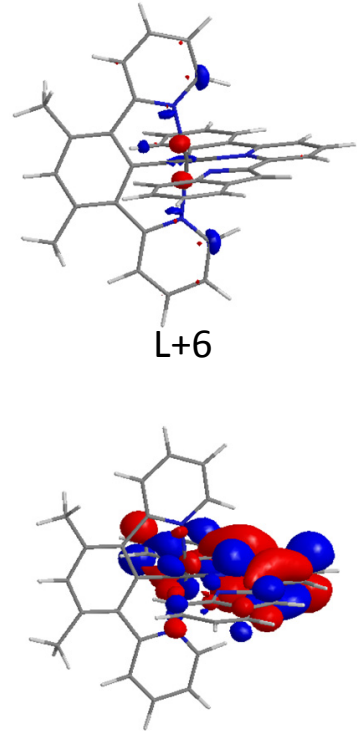

$\mathrm{L}+2$

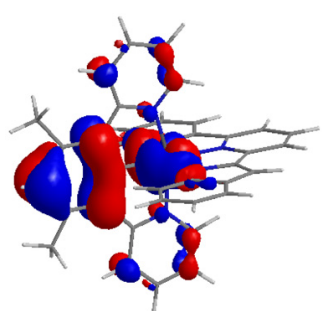

$\mathrm{H}-1$

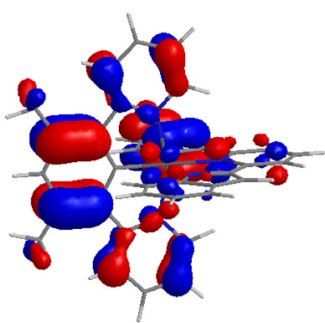

H-5

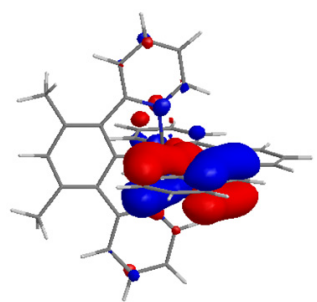

H-9

Figure S11. Frontier molecular orbitals of complex 9 (isovalue $0.03 \mathrm{au}$ ). 


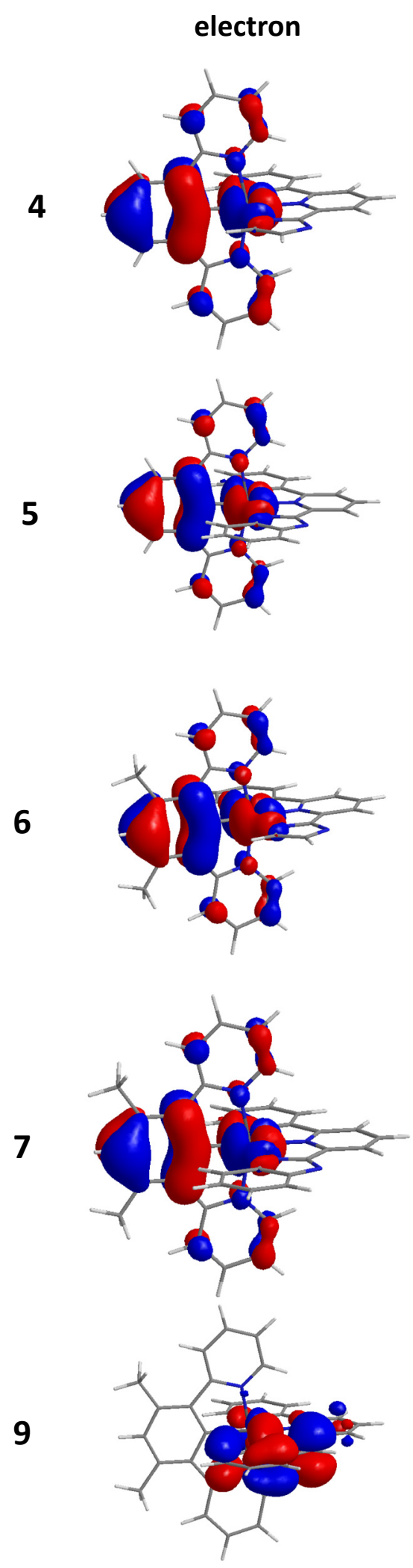

a
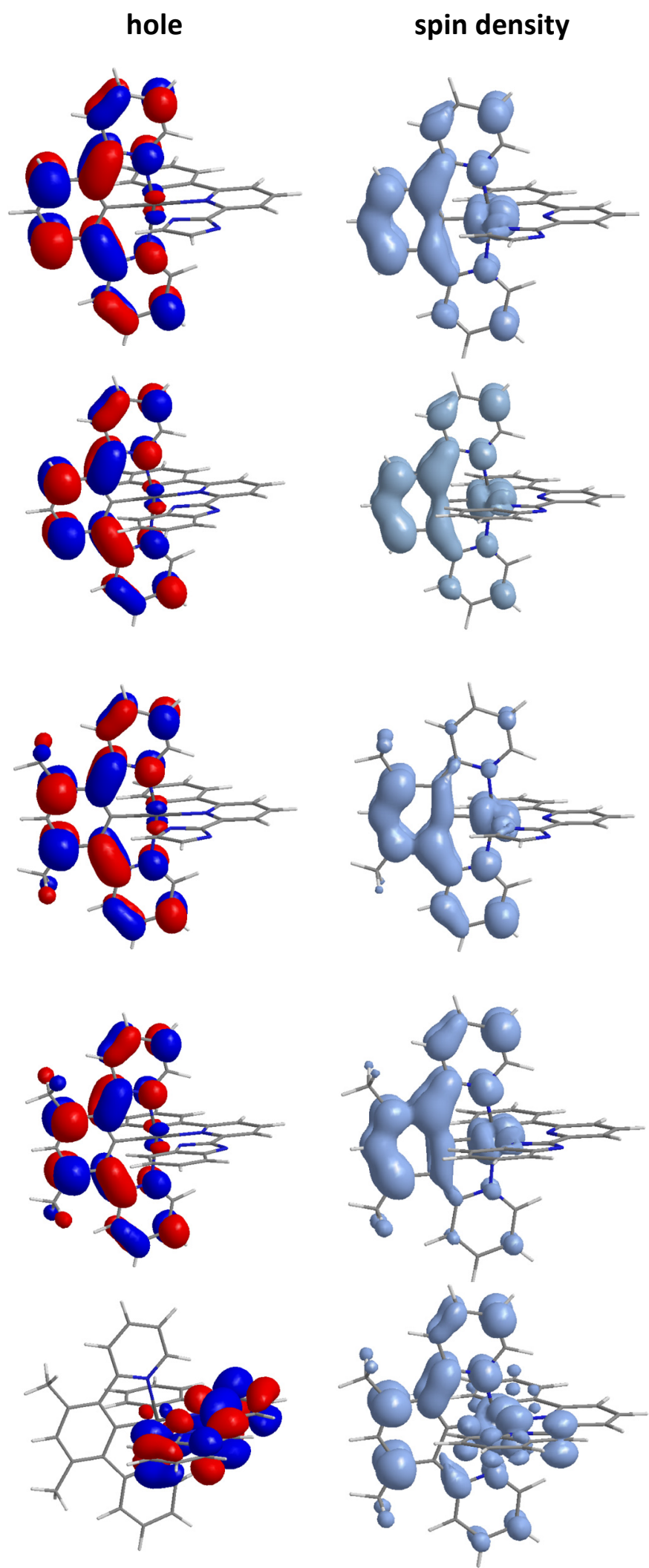

b
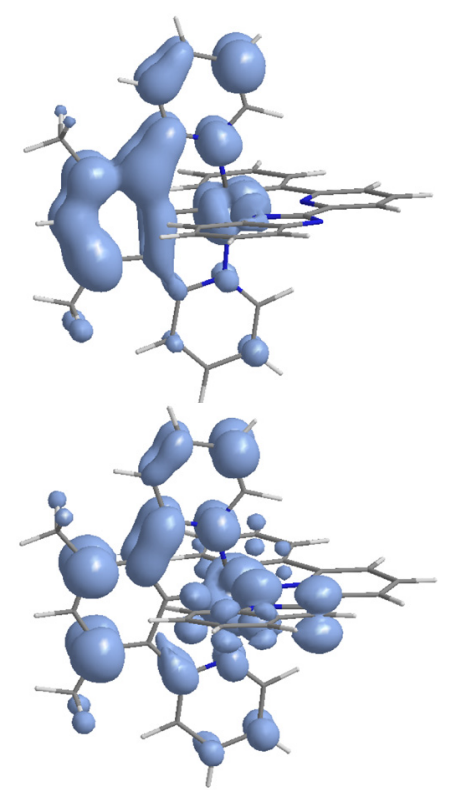

C

Figure S12. Natural transition orbitals (NTOs) (isovalue $=0.03$ ) for complexes 4-7 and 9 illustrating the nature of optically active triplet excited state $\mathrm{T}_{1}(\mathrm{a}$ and $\mathrm{b}$ ) and spin-density calculated (isovalue $=$ 0.002 ) for the $T_{1}$ excited state (c). 
Normalized Excitation and Emission Spectra of Complexes 4-7 and 9

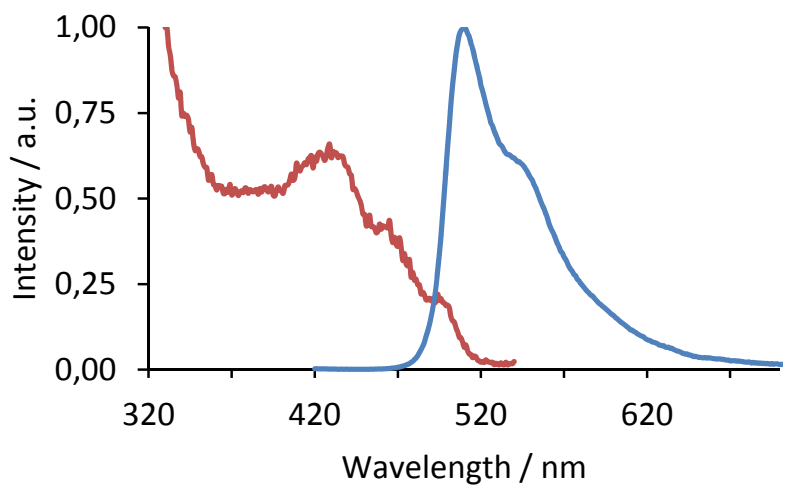

Figure S13. Normalized excitation (red line) and emission (blue line) spectra of complex 4 in PMMA film (5 $\mathrm{wt} \%)$ at $298 \mathrm{~K}$.

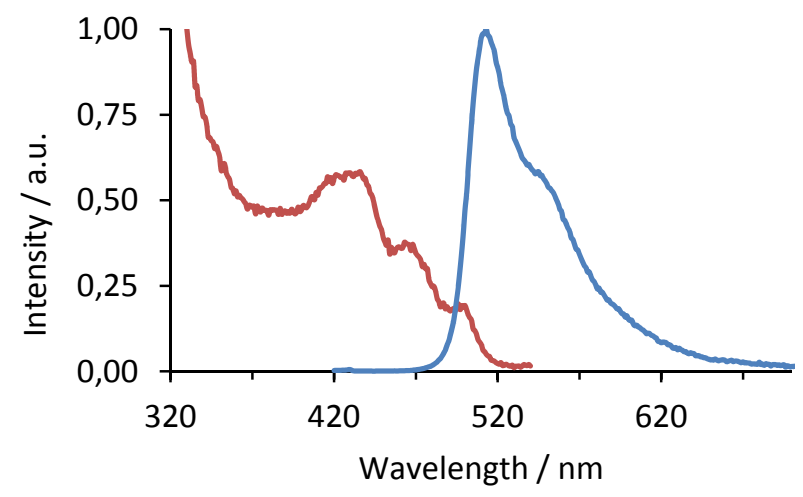

Figure S14. Normalized excitation (red line) and emission (blue line) spectra of complex 4 in a $2.0 \mathrm{x}$ $10^{-5} \mathrm{M}$ solution in 2-MeTHF at $298 \mathrm{~K}$.

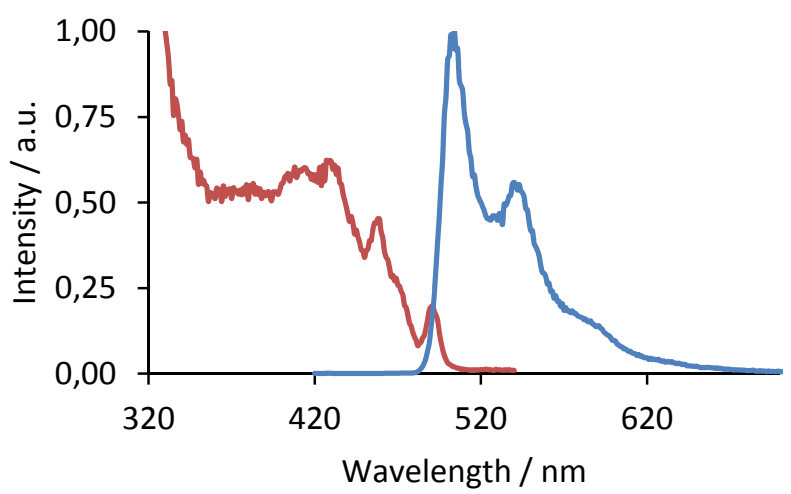

Figure S15. Normalized excitation (red line) and emission (blue line) spectra of complex 4 in a $2.0 \mathrm{x}$ $10^{-5} \mathrm{M}$ solution in 2-MeTHF at $77 \mathrm{~K}$. 


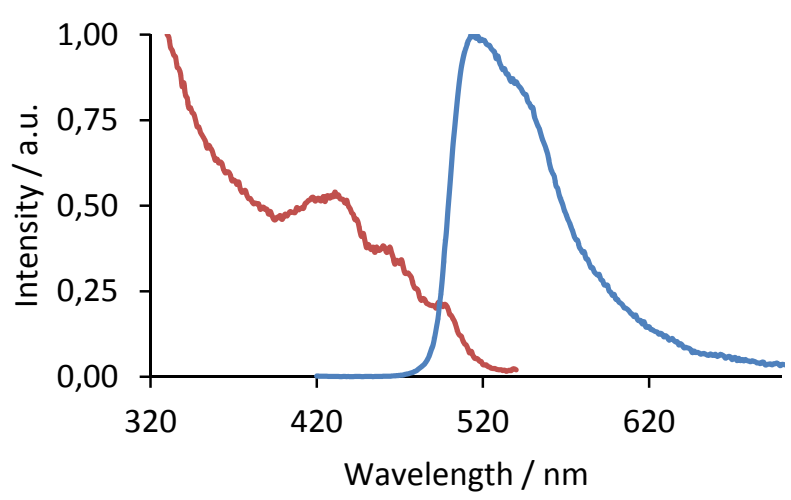

Figure S16. Normalized excitation (red line) and emission (blue line) spectra of complex 5 in PMMA film (5 wt\%) at $298 \mathrm{~K}$.

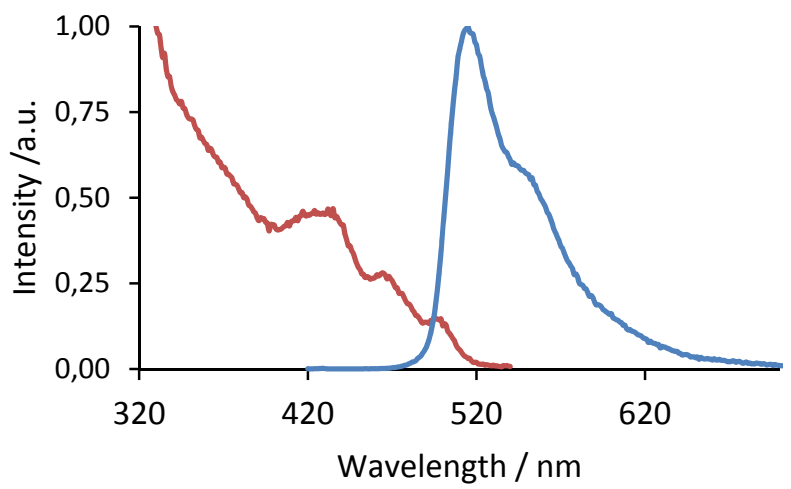

Figure S17. Normalized excitation (red line) and emission (blue line) spectra of complex 5 in a $2.0 \mathrm{x}$ $10^{-5} \mathrm{M}$ solution in $2-\mathrm{MeTHF}$ at $298 \mathrm{~K}$.

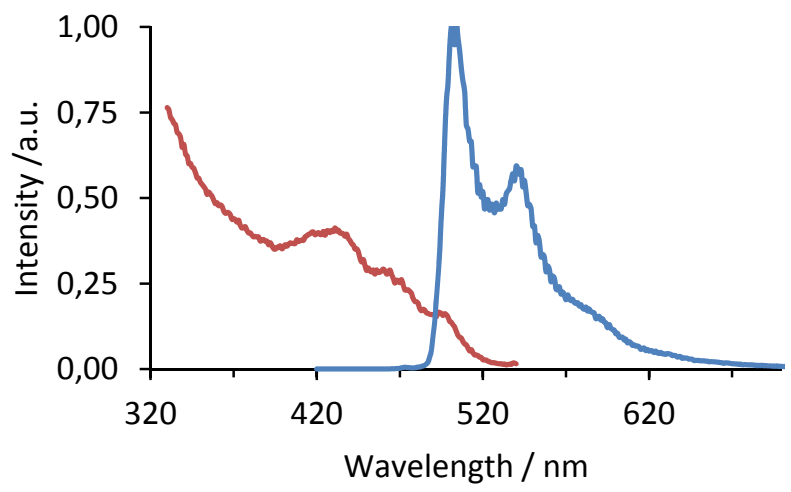

Figure S18. Normalized excitation (red line) and emission (blue line) spectra of complex 5 in a $2.0 \mathrm{x}$ $10^{-5} \mathrm{M}$ solution in 2-MeTHF at $77 \mathrm{~K}$. 


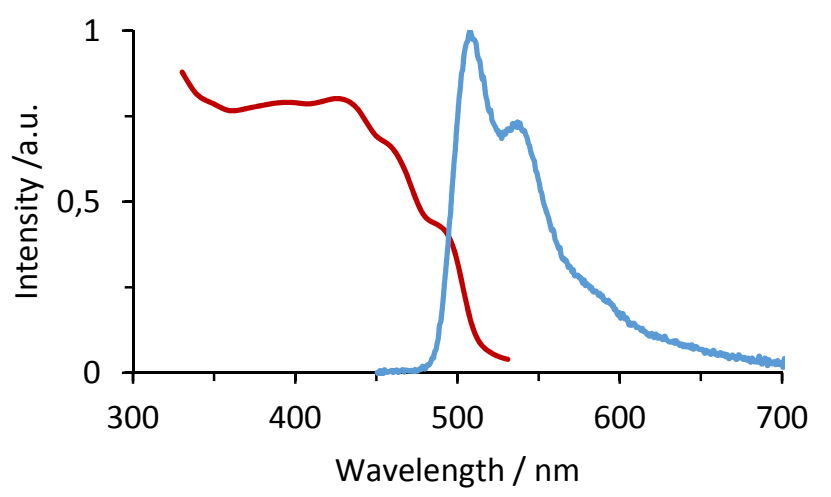

Figure S19. Normalized excitation (red line) and emission (blue line) spectra of complex 6 in PMMA film (5 wt\%) at $298 \mathrm{~K}$.

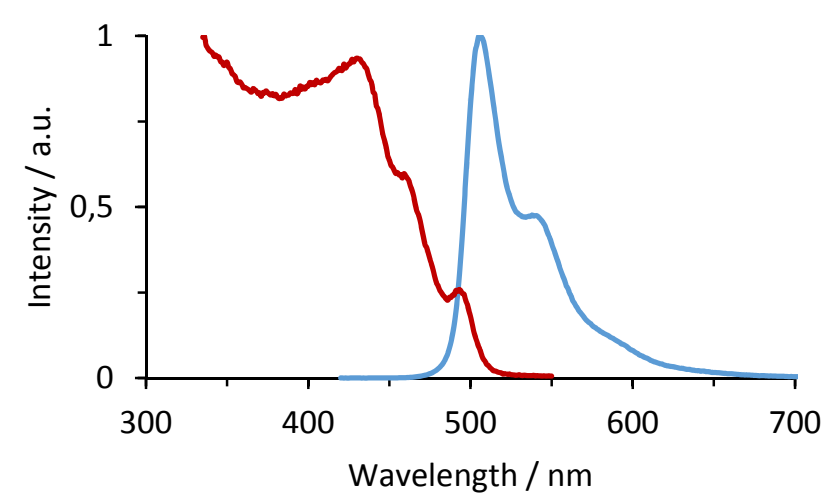

Figure S20. Normalized excitation (red line) and emission (blue line) spectra of complex 6 in a $2.5 \mathrm{x}$ $10^{-5} \mathrm{M}$ solution in 2-MeTHF at $298 \mathrm{~K}$.

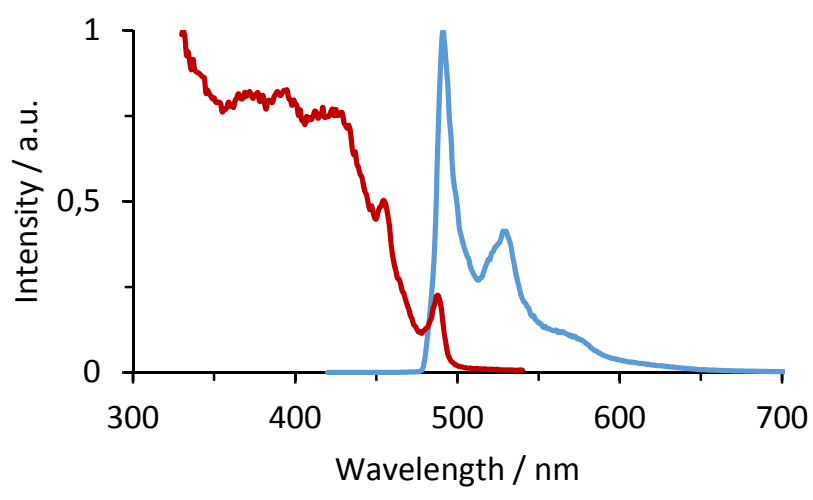

Figure S21. Normalized excitation (red line) and emission (blue line) spectra of complex 6 in a $2.5 \mathrm{x}$ $10^{-5} \mathrm{M}$ solution in 2-MeTHF at $77 \mathrm{~K}$. 


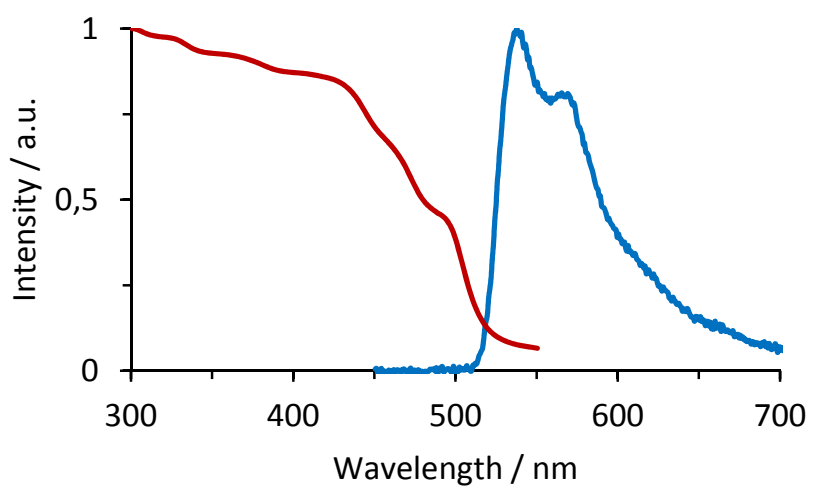

Figure S22. Normalized excitation (red line) and emission (blue line) spectra of complex 7 in PMMA film (5 wt\%) at $298 \mathrm{~K}$.

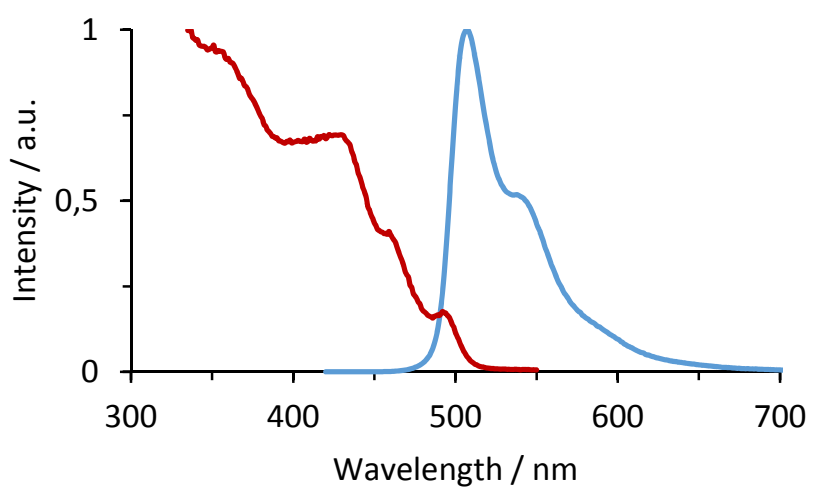

Figure S23. Normalized excitation (red line) and emission (blue line) spectra of complex 7 in a $2.5 \mathrm{x}$ $10^{-5} \mathrm{M}$ solution in 2-MeTHF at $298 \mathrm{~K}$.

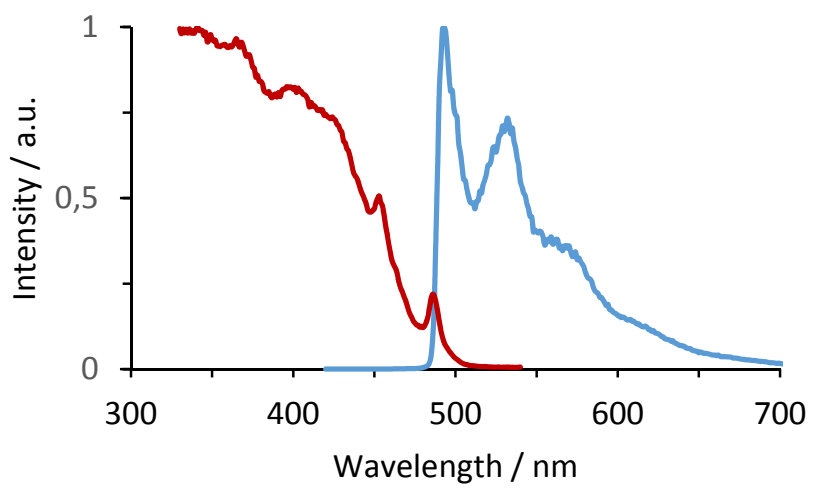

Figure S24. Normalized excitation (red line) and emission (blue line) spectra of complex 7 in a $2.5 \mathrm{x}$ $10^{-5} \mathrm{M}$ solution in 2-MeTHF at $77 \mathrm{~K}$. 


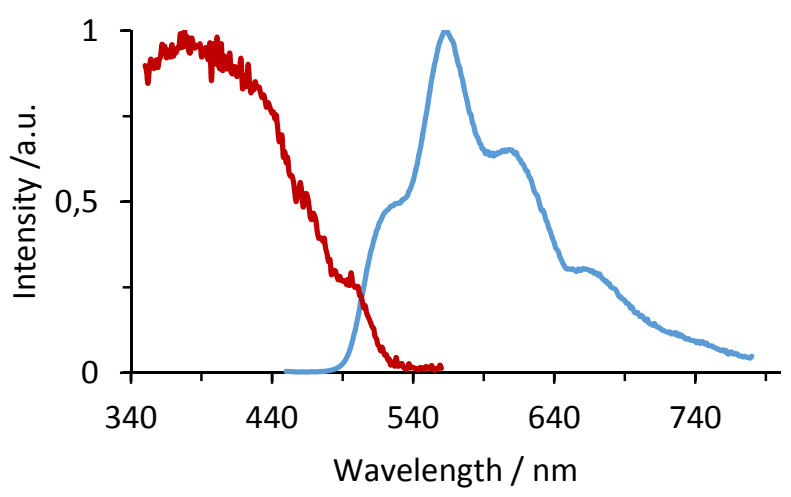

Figure S25. Normalized excitation (red line) and emission (blue line) spectra of complex 9 in PMMA film (5 wt\%) at $298 \mathrm{~K}$.

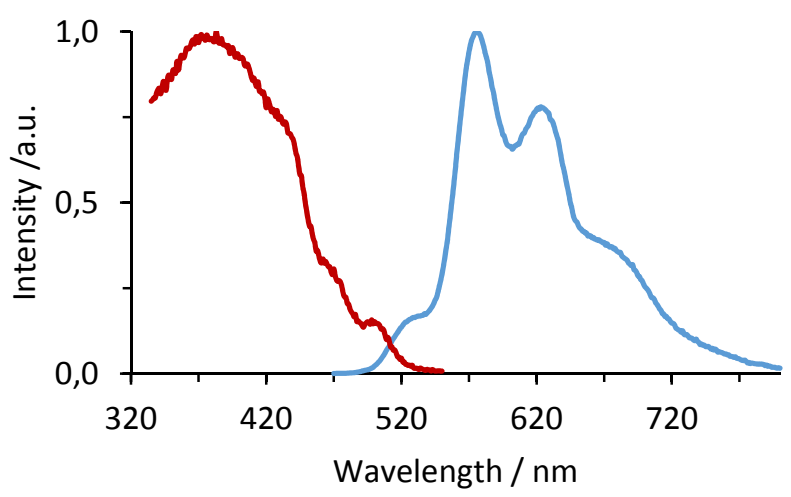

Figure S26. Normalized excitation (red line) and emission (blue line) spectra of complex 9 in a $2.5 \mathrm{x}$ $10^{-5} \mathrm{M}$ solution in 2-MeTHF at $298 \mathrm{~K}$.

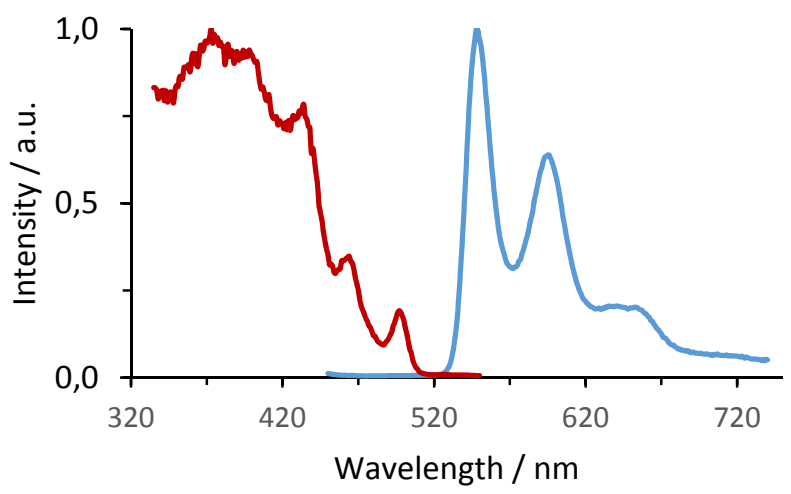

Figure S27. Normalized excitation (red line) and emission (blue line) spectra of complex 9 in a $2.5 \mathrm{x}$ $10^{-5} \mathrm{M}$ solution in 2-MeTHF at $77 \mathrm{~K}$. 


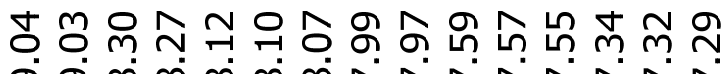

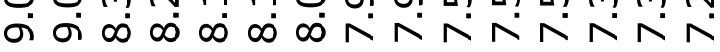
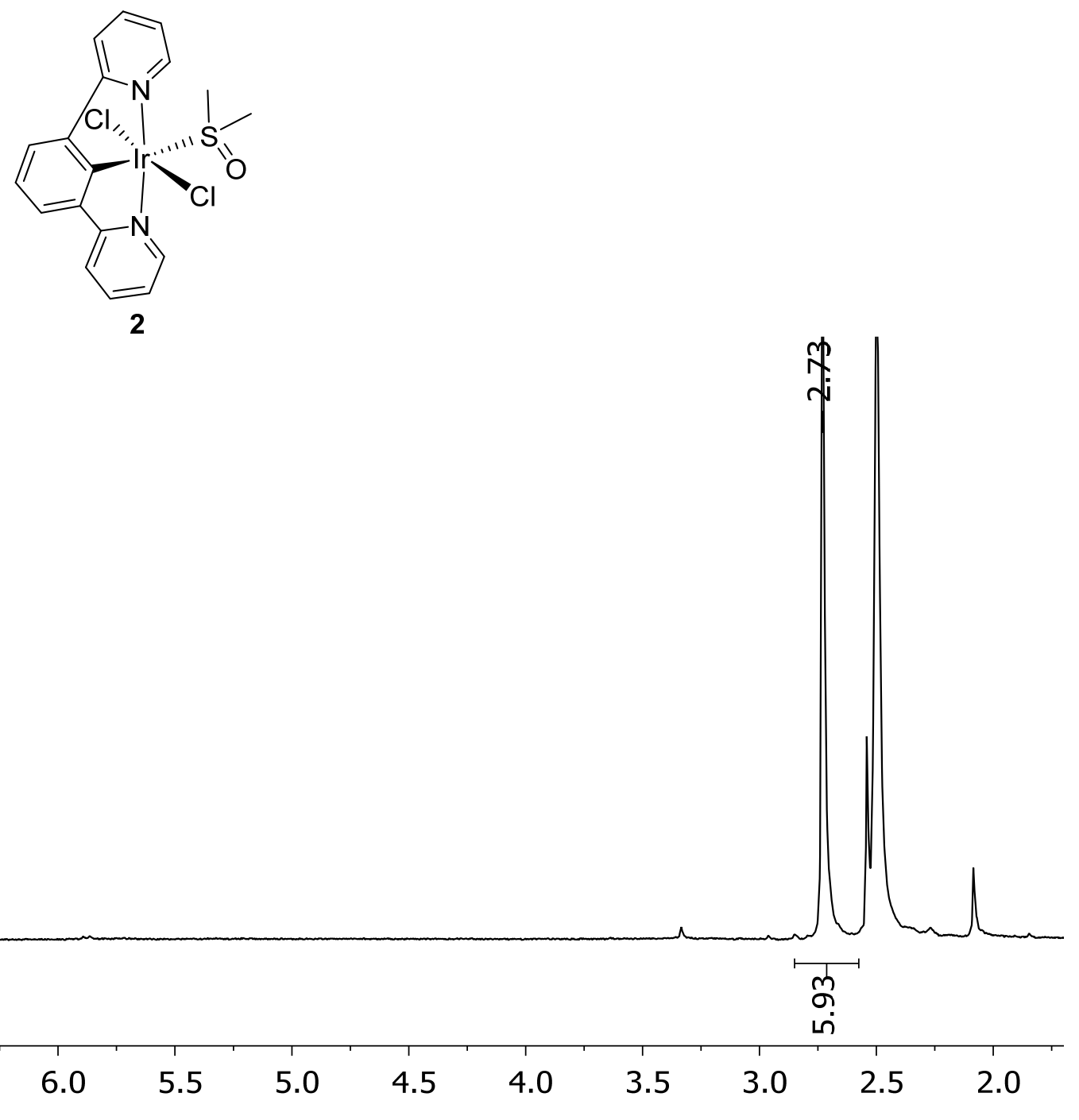

Figure S28. ${ }^{1}$ H NMR (300 MHz, DMSO- $d_{6}$, $\left.298 \mathrm{~K}\right)$ spectrum of complex 2. 


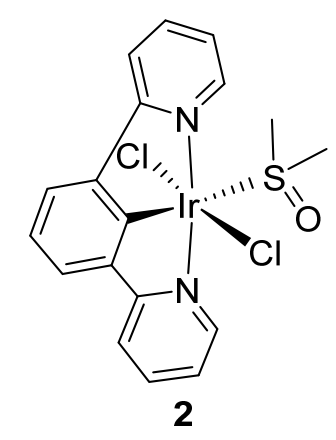

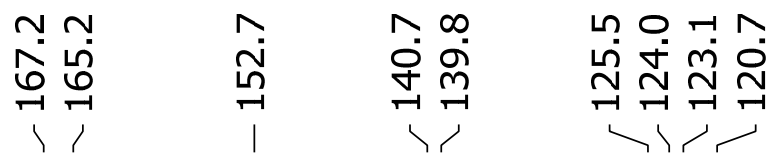

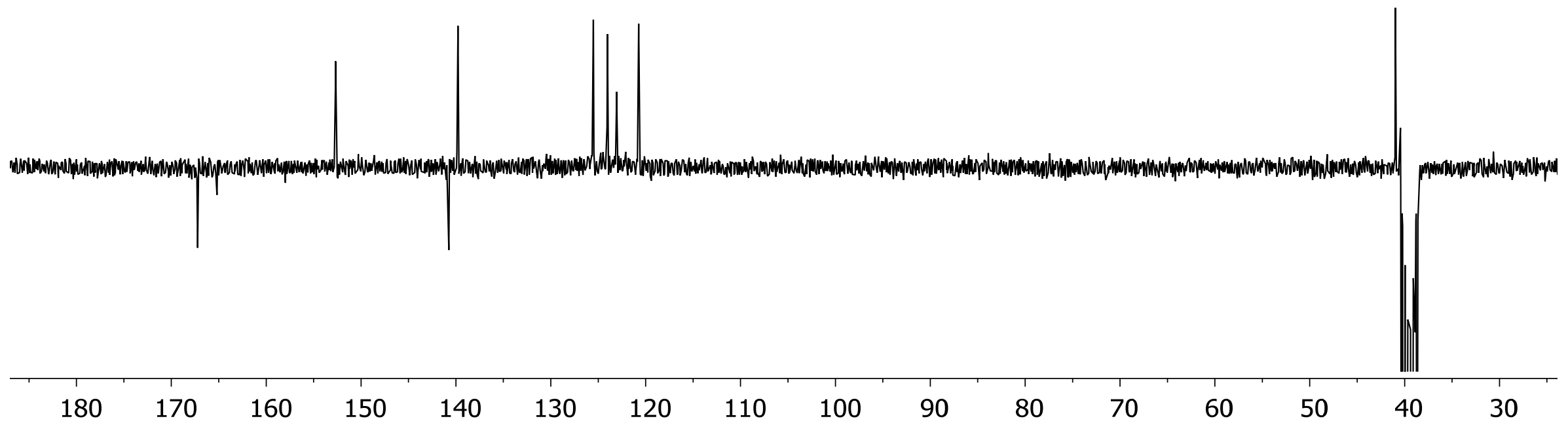

Figure S29. ${ }^{13} \mathrm{C}\left\{{ }^{1} \mathrm{H}\right\}$-APT NMR(75 MHz, DMSO- $\left.d_{6}, 298 \mathrm{~K}\right)$ spectrum of complex 2. 


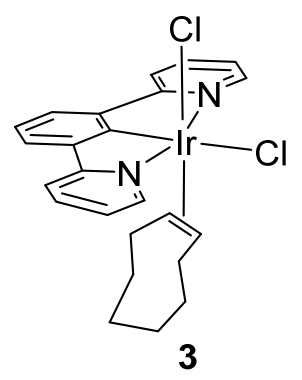

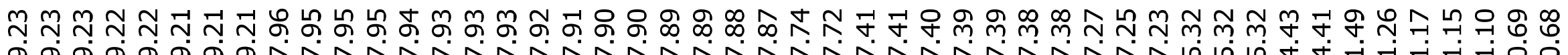

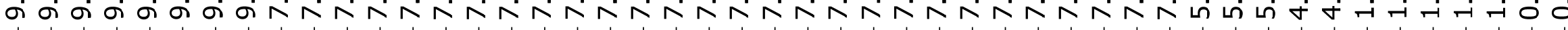

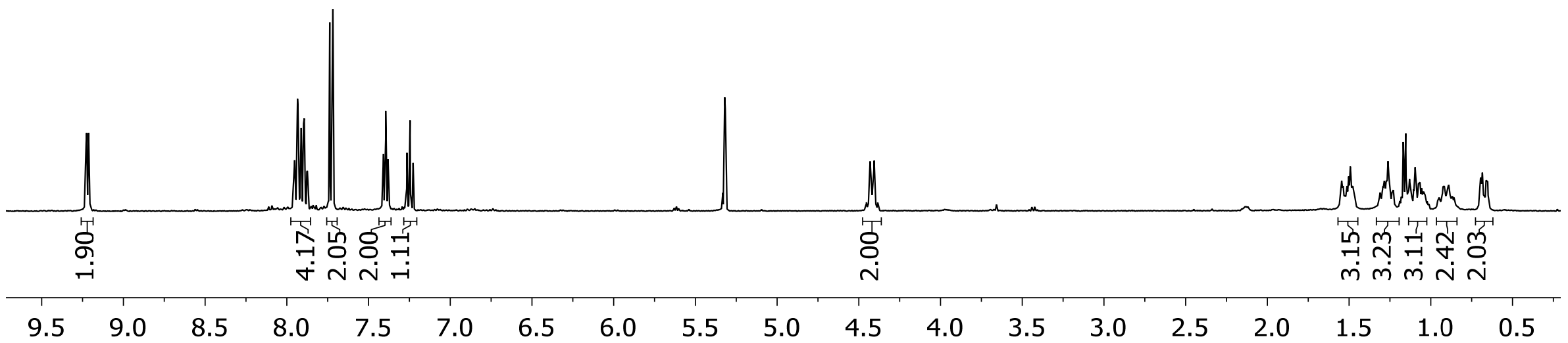

Figure S30. ${ }^{1} \mathrm{H}$ NMR (400 MHz, $\left.\mathrm{C} \mathrm{D}_{2} \mathrm{Cl}_{2}, 298 \mathrm{~K}\right)$ spectrum of complex 3. 

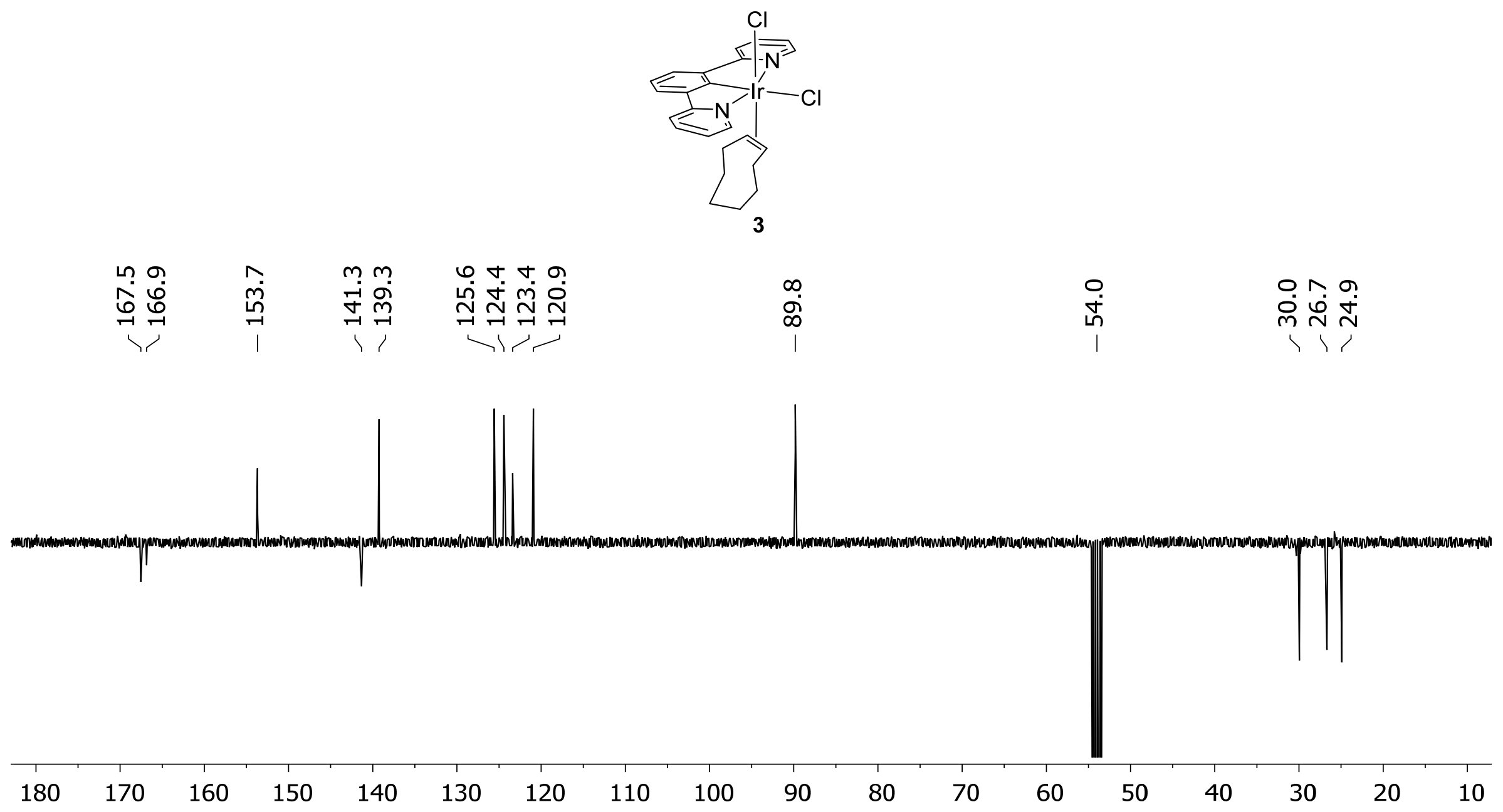

Figure S31. ${ }^{13} \mathrm{C}\left\{{ }^{1} \mathrm{H}\right\}$-APT NMR(100 MHz, $\left.\mathrm{CD}_{2} \mathrm{Cl}_{2}, 233 \mathrm{~K}\right)$ spectrum of complex 3. 


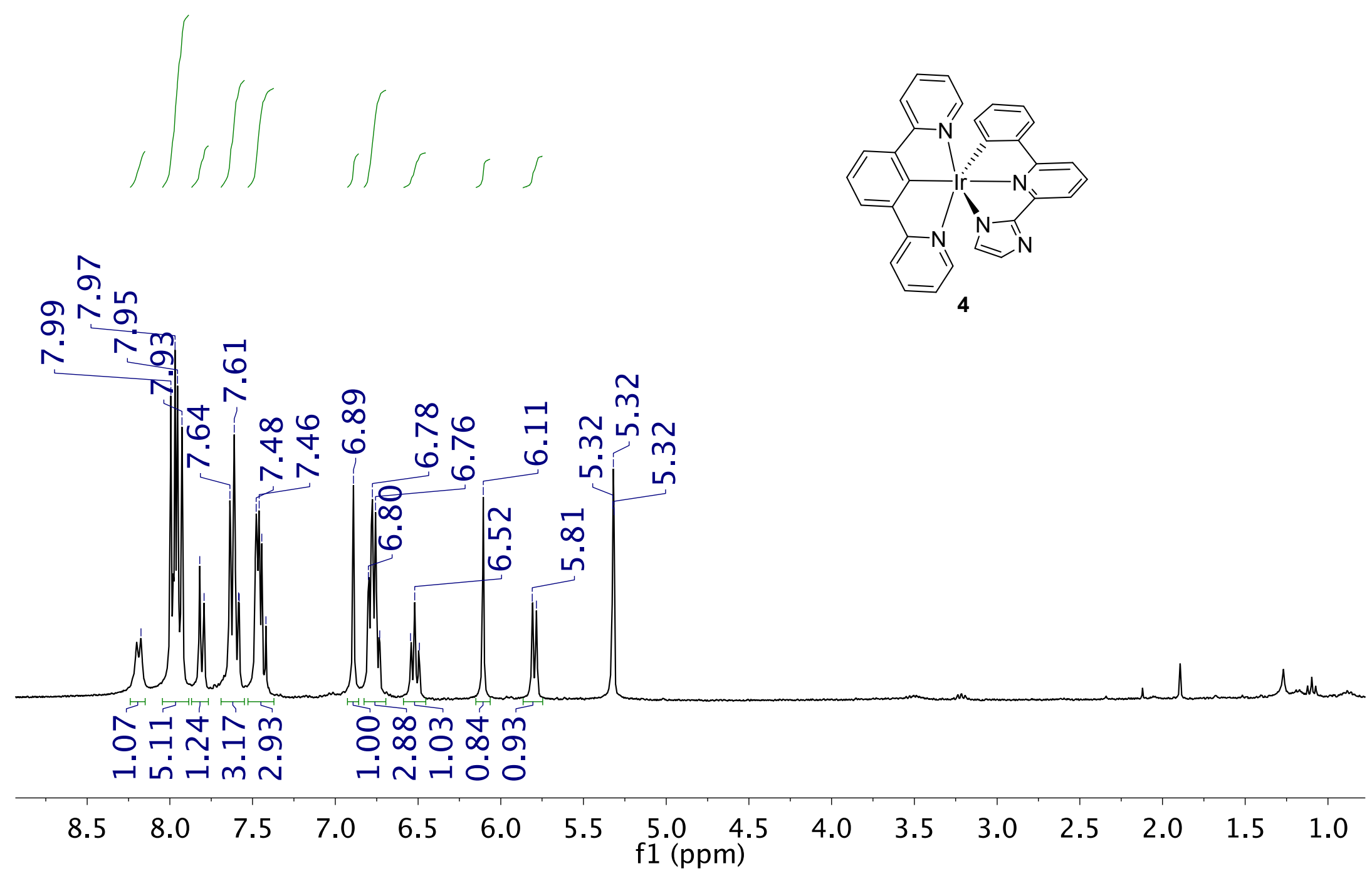

Figure S32. ${ }^{1} \mathrm{H}$ NMR $\left(300 \mathrm{MHz}, \mathrm{CD}_{2} \mathrm{Cl}_{2}, 298 \mathrm{~K}\right)$ spectrum of complex 4. 


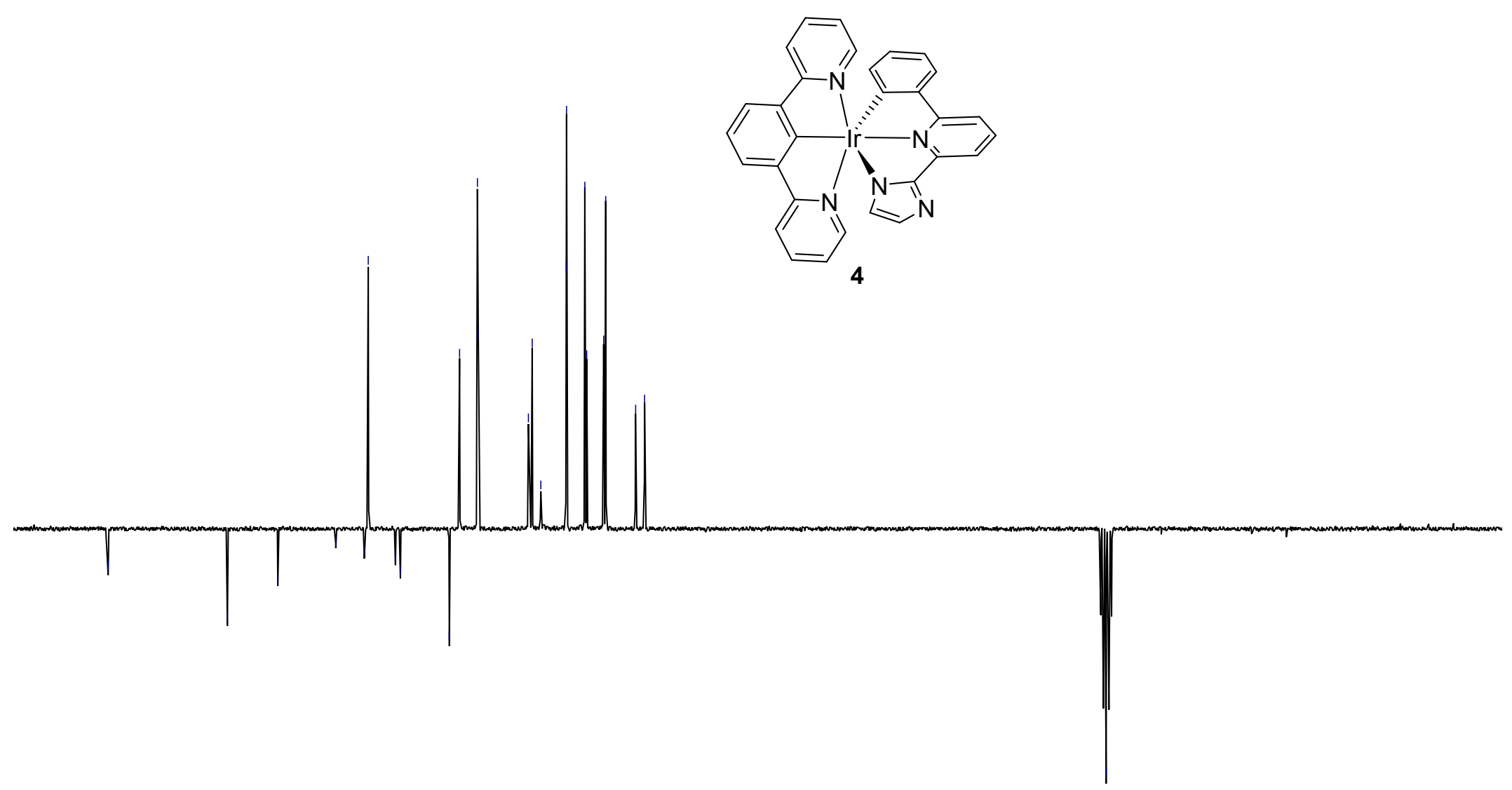

$\begin{array}{llllllllll}190 & 180 & 170 & 160 & 150 & 140 & 130 & 120 & 110 & 100\end{array}$

Figure S33. ${ }^{13} \mathrm{C}\left\{{ }^{1} \mathrm{H}\right\}$-APT NMR $\left(75 \mathrm{MHz}, \mathrm{CD}_{2} \mathrm{Cl}_{2}, 298 \mathrm{~K}\right)$ spectrum of complex 4. 


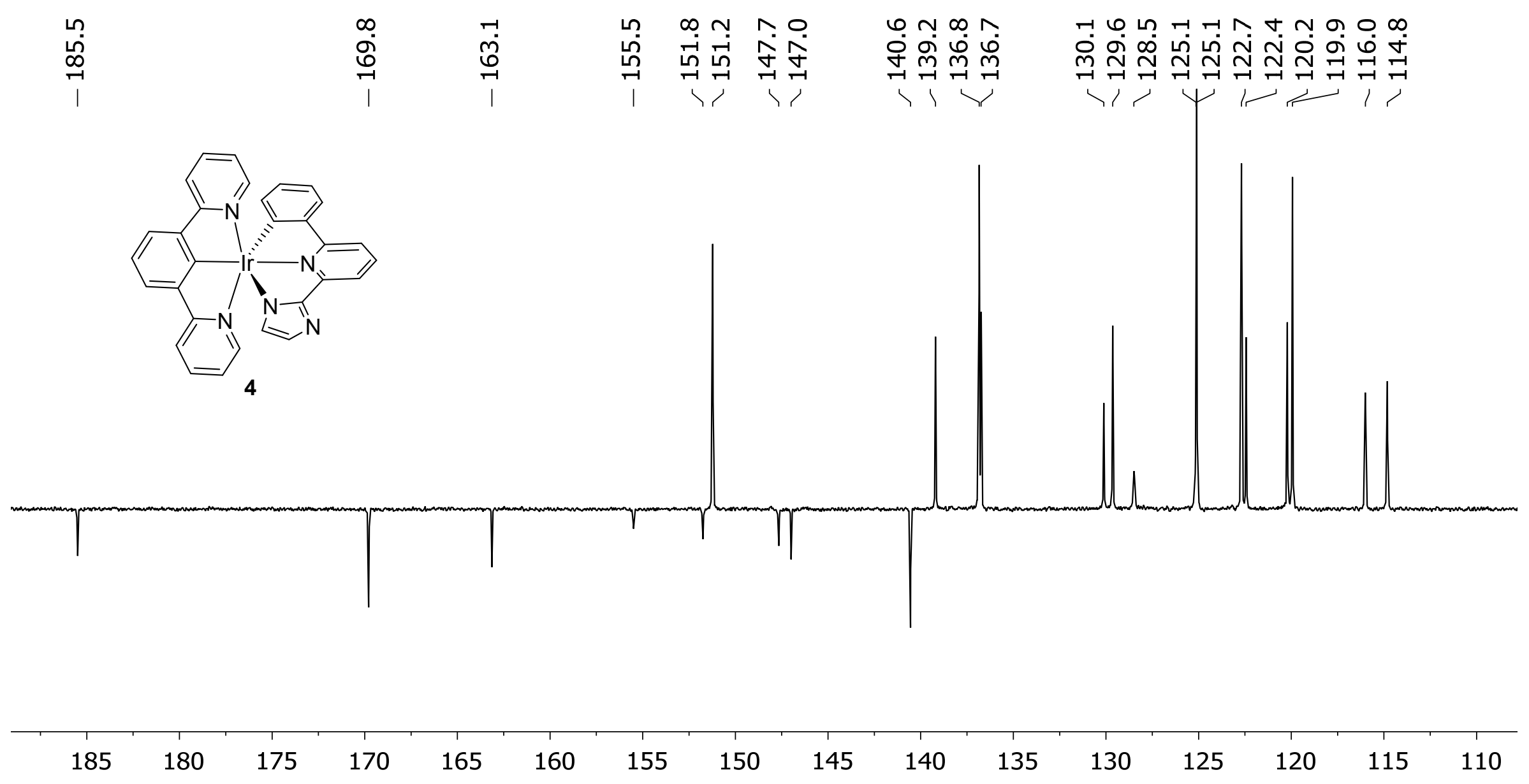

Figure S34. Aromatic region of the ${ }^{13} \mathrm{C}\left\{{ }^{1} \mathrm{H}\right\}$-APT NMR $\left(75 \mathrm{MHz}, \mathrm{CD}_{2} \mathrm{Cl}_{2}, 298 \mathrm{~K}\right)$ spectrum of complex 4. 


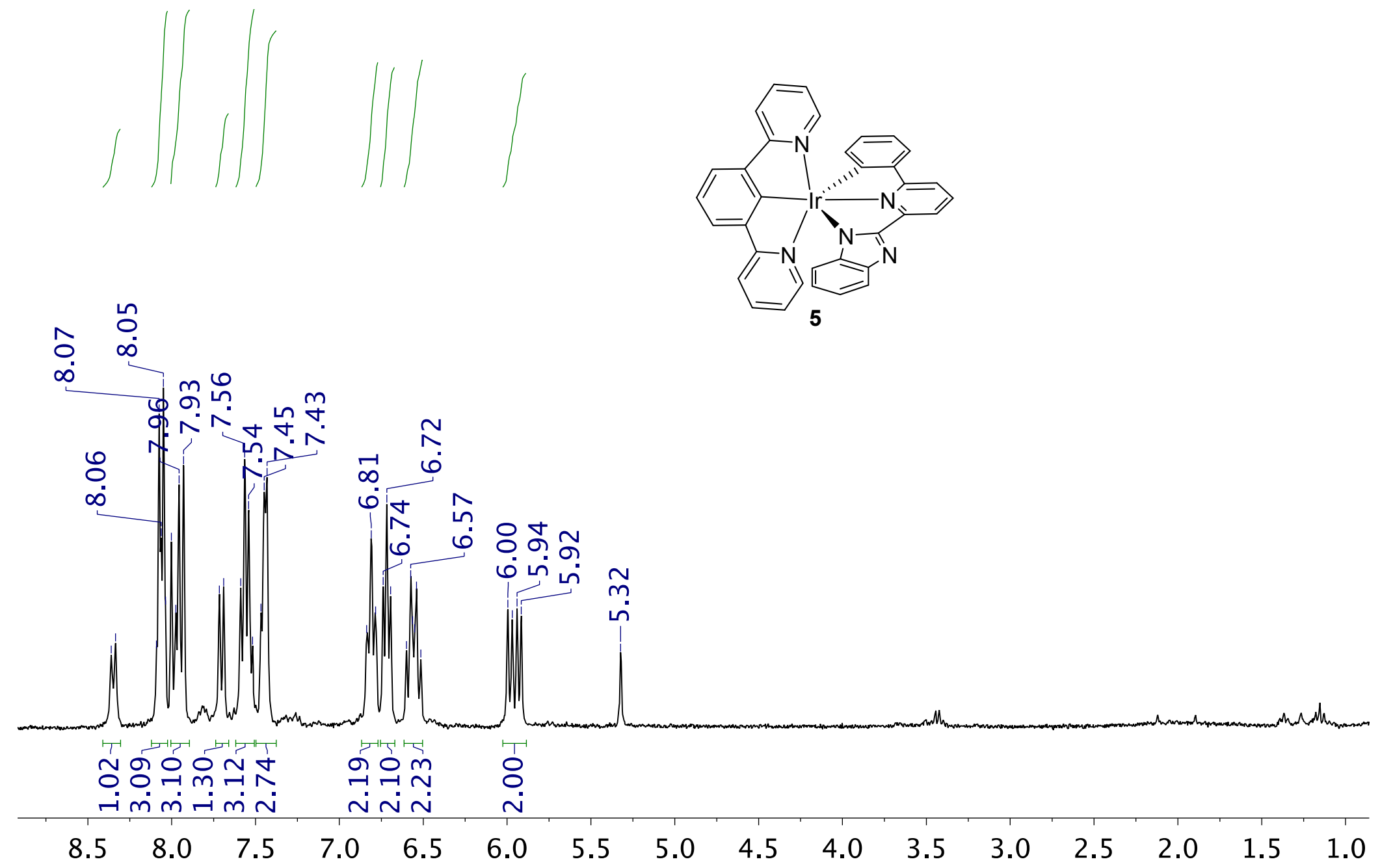

Figure S35. ${ }^{1} \mathrm{H}$ NMR $\left(300 \mathrm{MHz}, \mathrm{CD}_{2} \mathrm{Cl}_{2}, 298 \mathrm{~K}\right)$ spectrum of complex 5. 


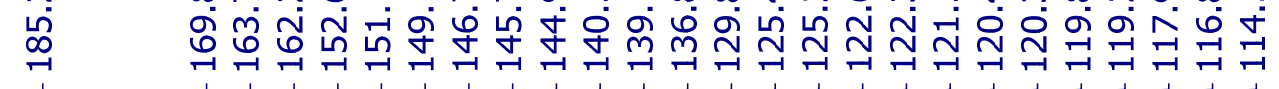

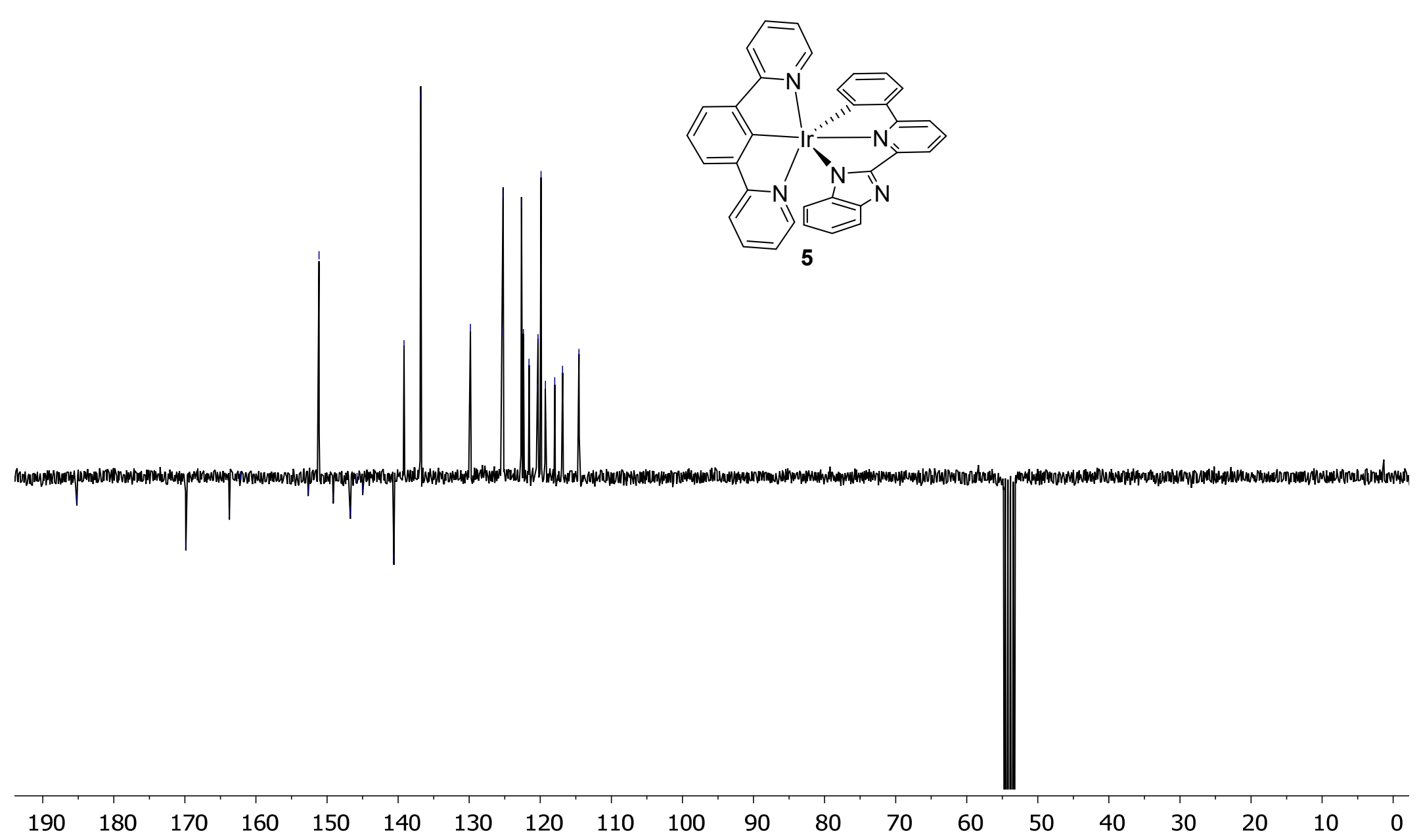

Figure S36. ${ }^{13} \mathrm{C}\left\{{ }^{1} \mathrm{H}\right\}$-APT NMR $\left(75 \mathrm{MHz}, \mathrm{CD}_{2} \mathrm{Cl}_{2}, 298 \mathrm{~K}\right)$ spectrum of complex 5 . 

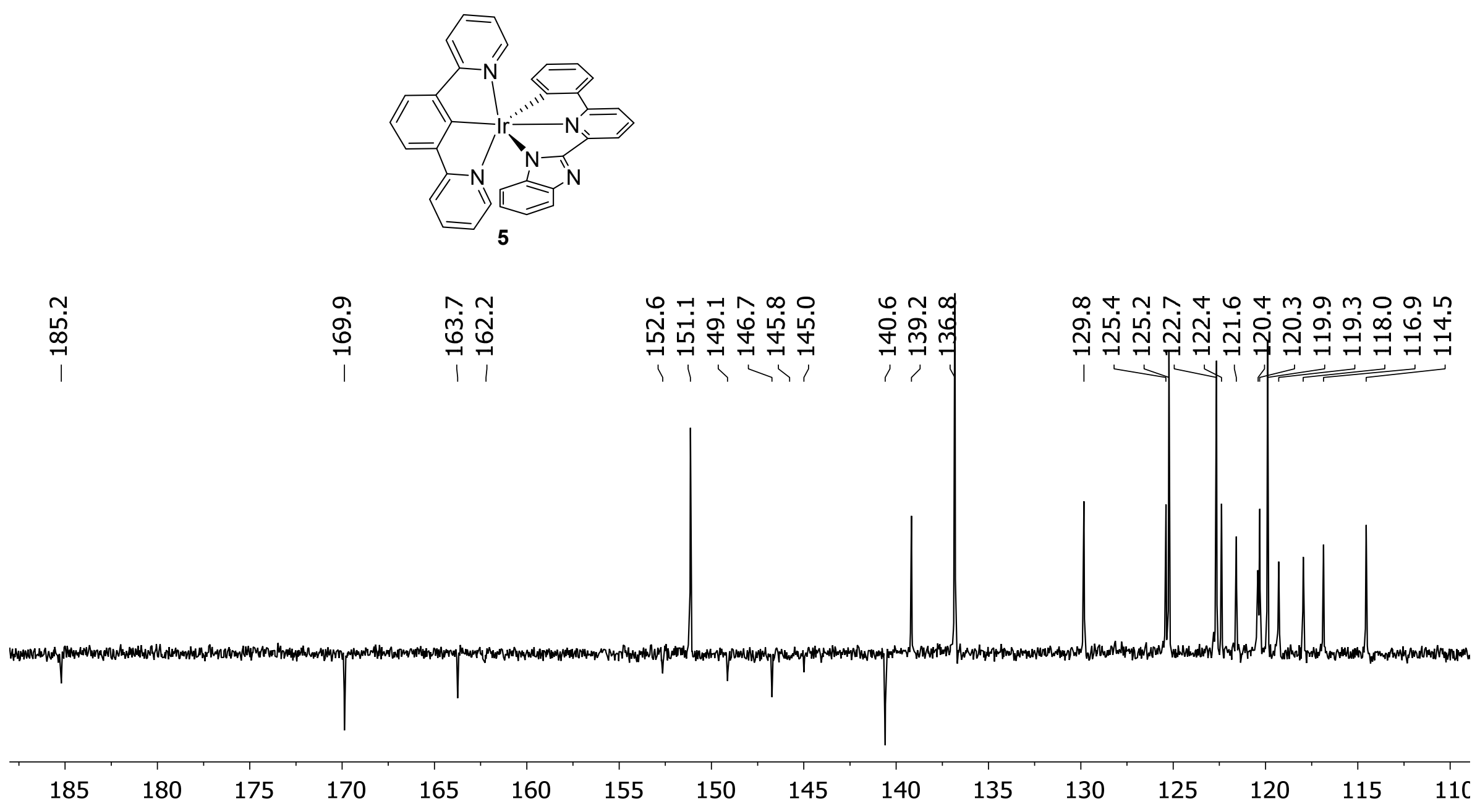

Figure S37. Aromatic region of the ${ }^{13} \mathrm{C}\left\{{ }^{1} \mathrm{H}\right\}$-APT NMR (75 MHz, $\left.\mathrm{CD}_{2} \mathrm{Cl}_{2}, 298 \mathrm{~K}\right)$ spectrum of complex 5. 

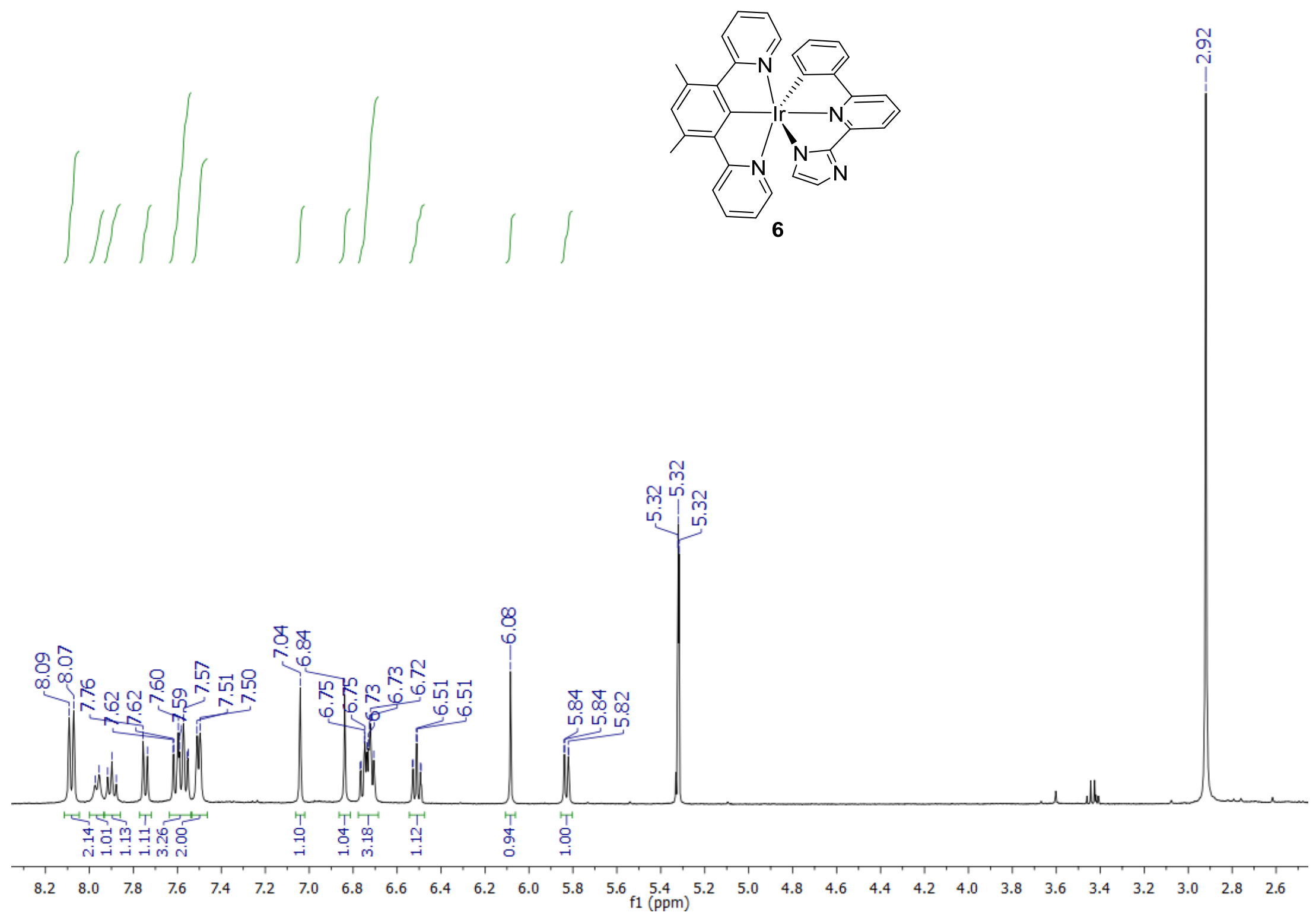

Figure S38. ${ }^{1} \mathrm{H}$ NMR (400 MHz, $\mathrm{CD}_{2} \mathrm{Cl}_{2}, 298 \mathrm{~K}$ ) spectrum of complex 6. 

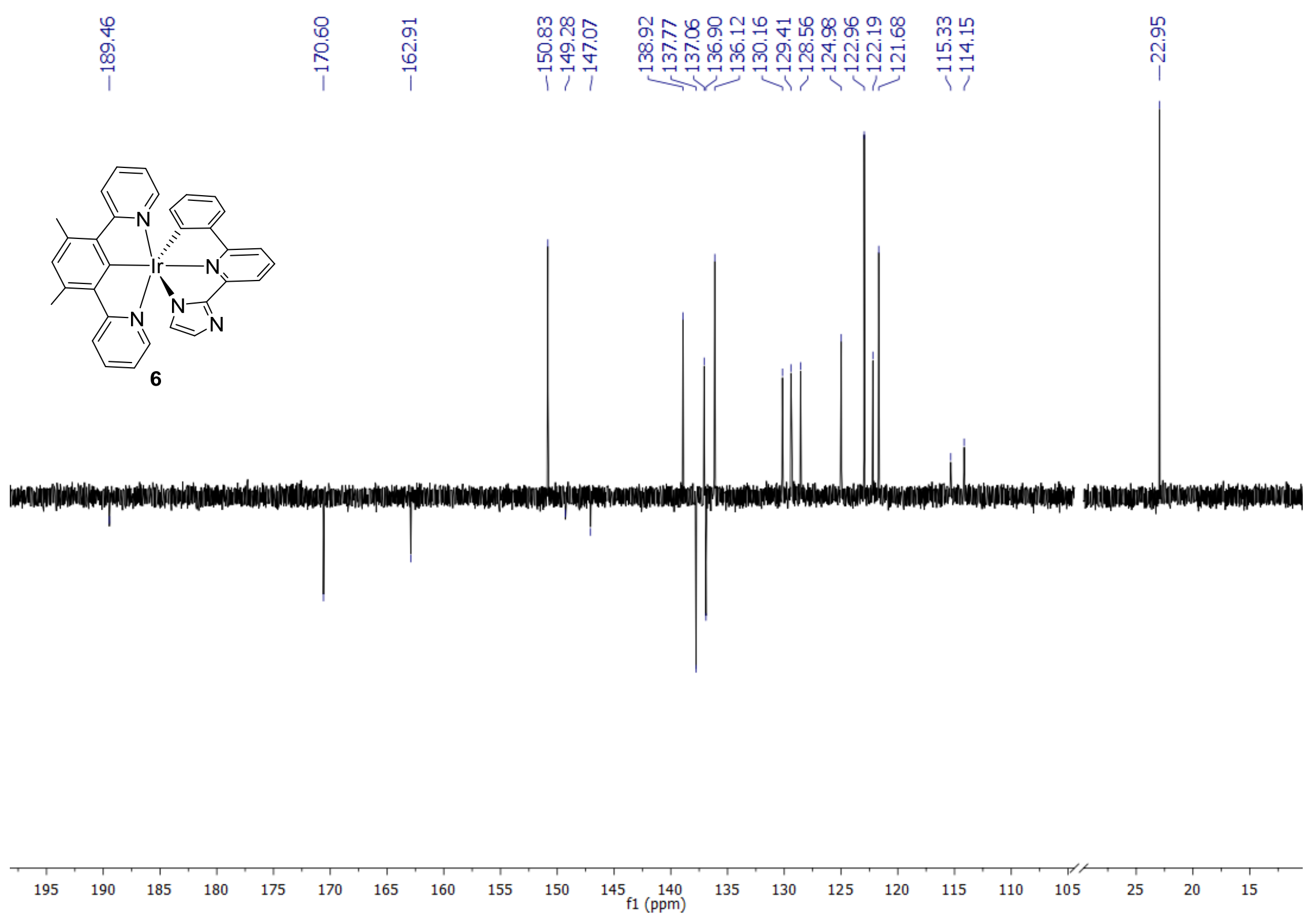

Figure S39. ${ }^{13} \mathrm{C}\left\{{ }^{1} \mathrm{H}\right\}$-APT NMR $\left(100 \mathrm{MHz}, \mathrm{CD}_{2} \mathrm{Cl}_{2}, 298 \mathrm{~K}\right)$ spectrum of complex 6. 

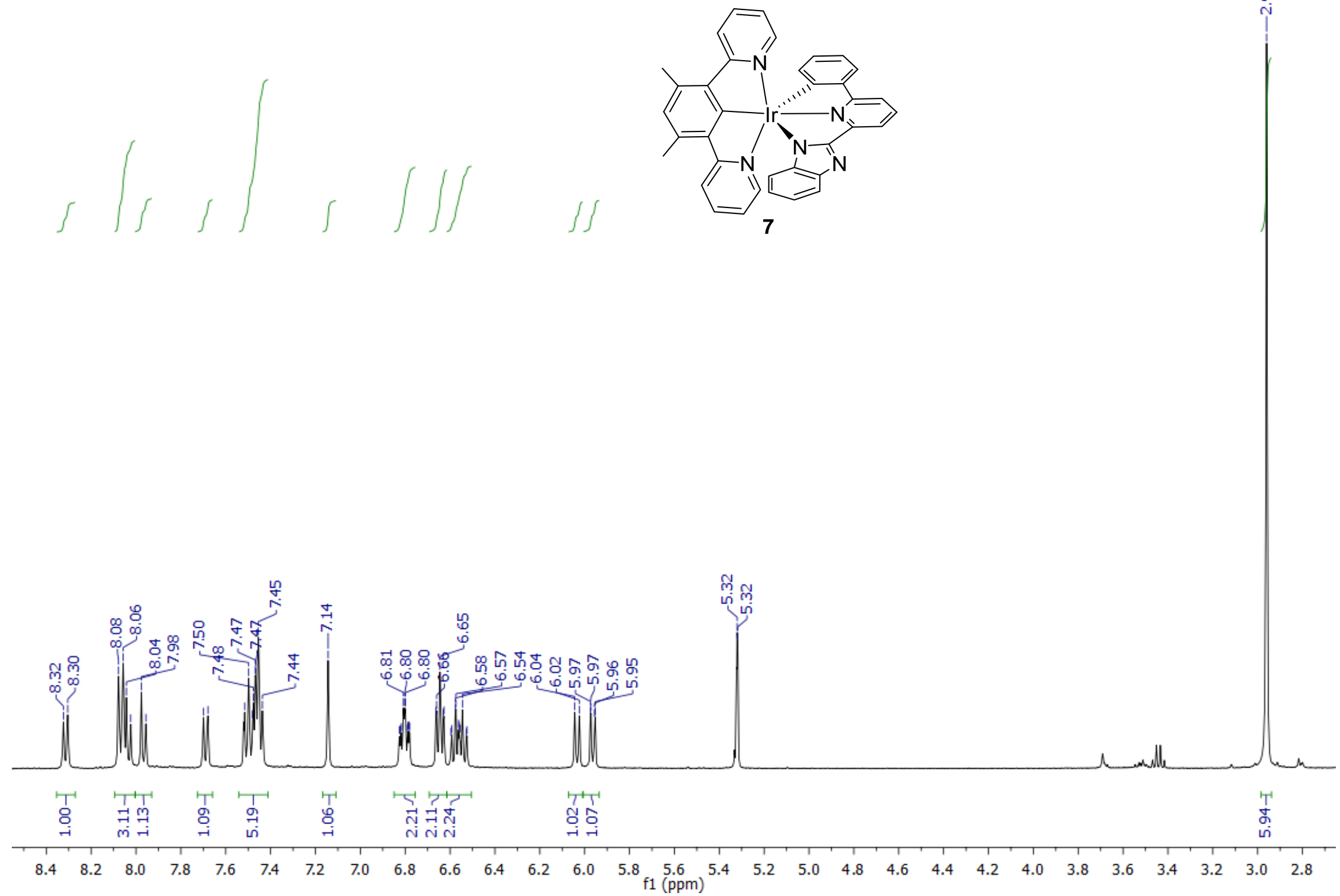

Figure S40. ${ }^{1} \mathrm{H}$ NMR (400 MHz, $\mathrm{CD}_{2} \mathrm{Cl}_{2}, 298 \mathrm{~K}$ ) spectrum of complex 7. 

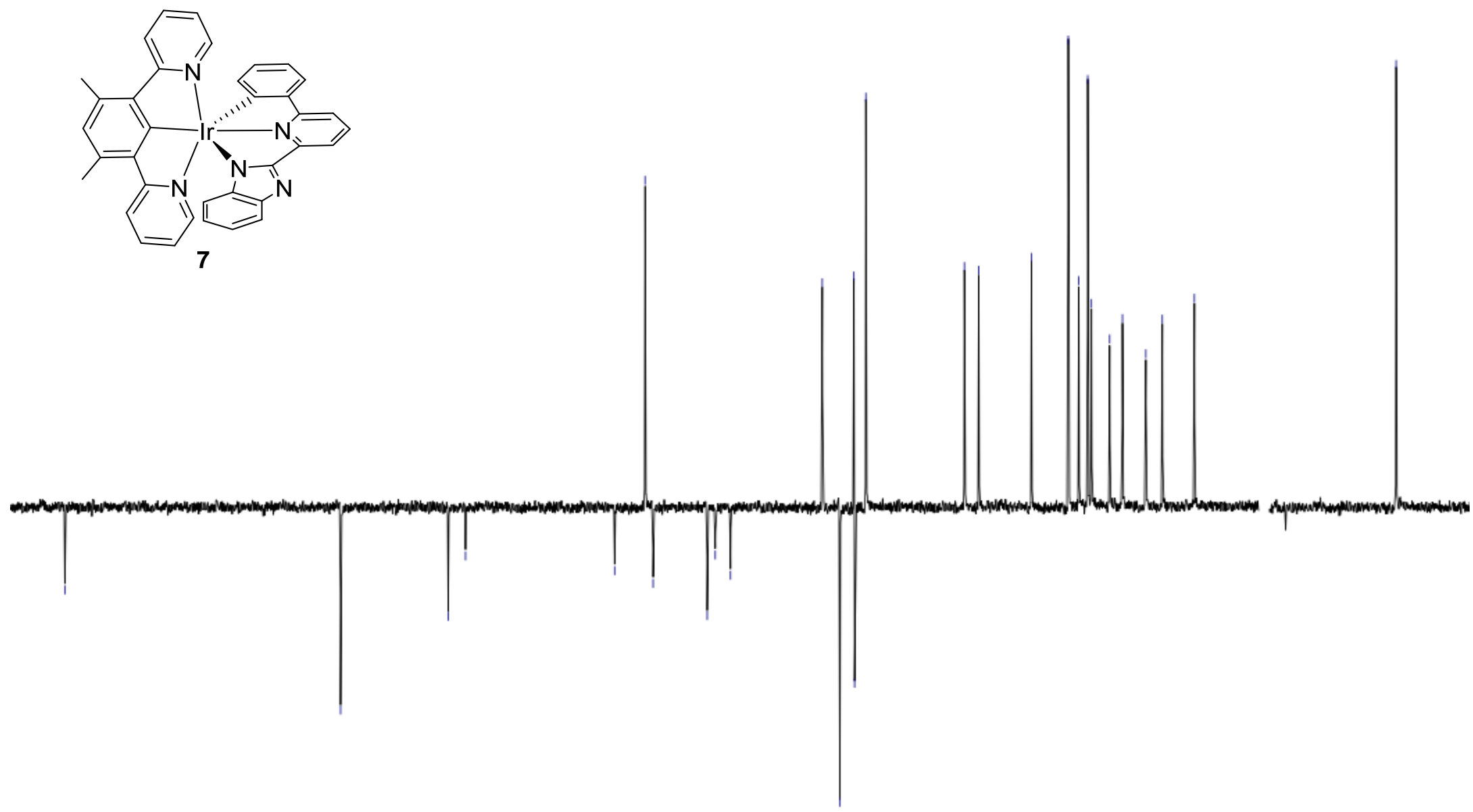

190

180

$75 \quad 170$

165

160

155

150

$145(\mathrm{ppm})$

130

125

120

115

30

$25 \quad 20$

Figure S41. ${ }^{13} \mathrm{C}\left\{{ }^{1} \mathrm{H}\right\}$-APT NMR $\left(100 \mathrm{MHz}, \mathrm{CD}_{2} \mathrm{Cl}_{2}, 298 \mathrm{~K}\right)$ spectrum of complex 7. 

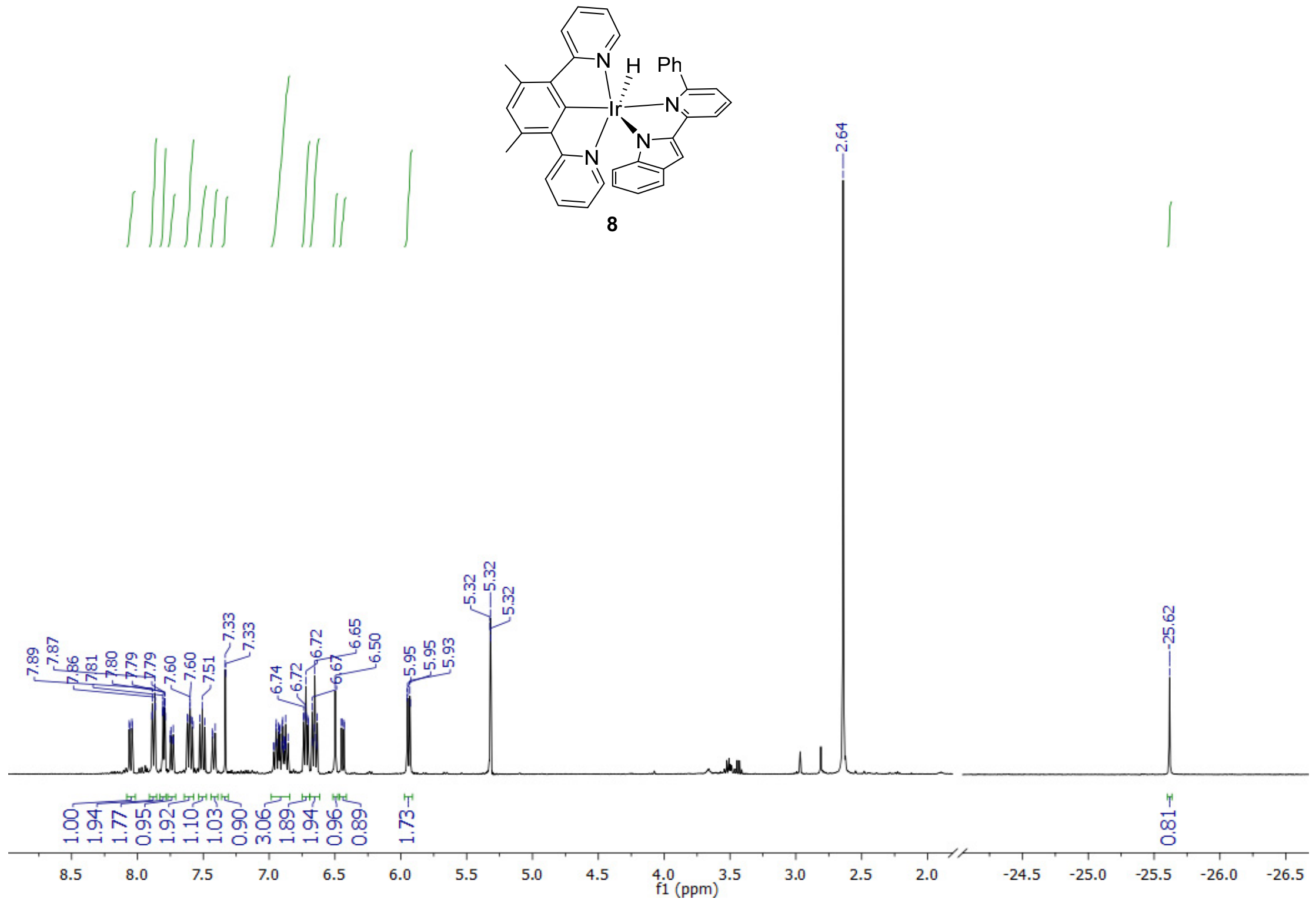

Figure S42. ${ }^{1} \mathrm{H}$ NMR (400 MHz, $\mathrm{CD}_{2} \mathrm{Cl}_{2}, 298 \mathrm{~K}$ ) spectrum of complex 8. 

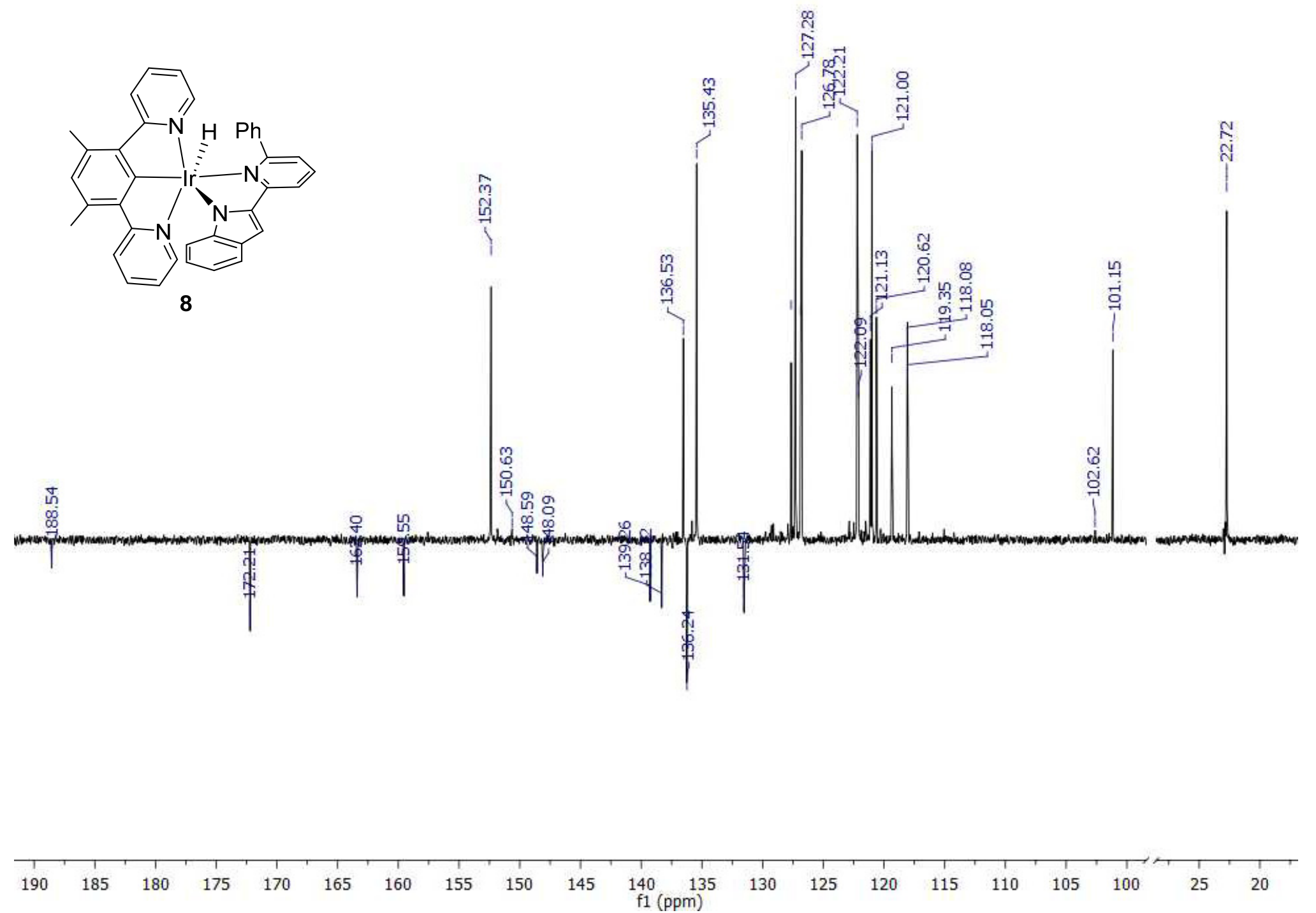

Figure S43. ${ }^{13} \mathrm{C}\left\{{ }^{1} \mathrm{H}\right\}$-APT NMR $\left(100 \mathrm{MHz}, \mathrm{CD}_{2} \mathrm{Cl}_{2}, 298 \mathrm{~K}\right)$ spectrum of complex 8. 

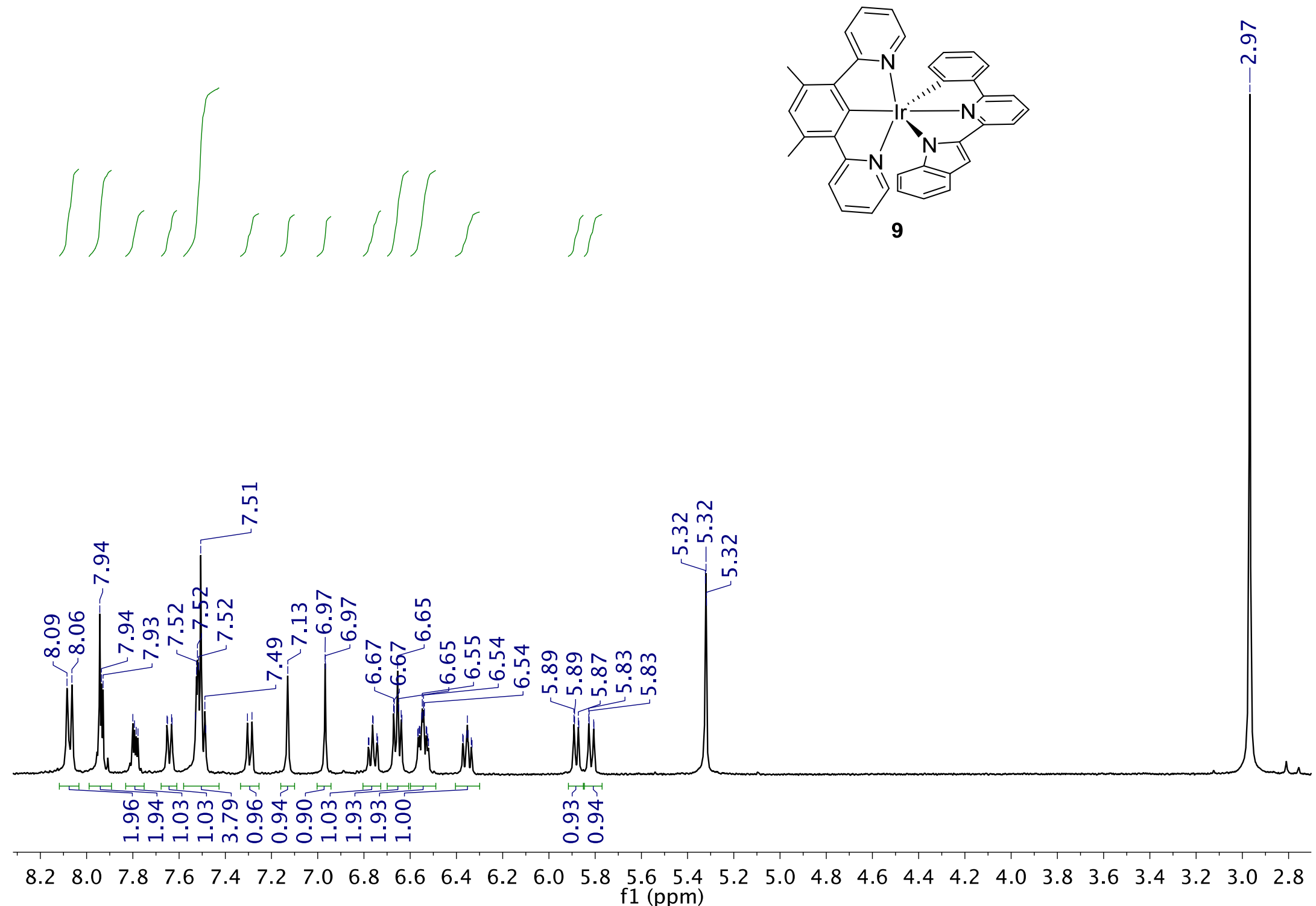

Figure S44. ${ }^{1} \mathrm{H}$ NMR (300 MHz, $\left.\mathrm{CD}_{2} \mathrm{Cl}_{2}, 298 \mathrm{~K}\right)$ spectrum of complex 9. 


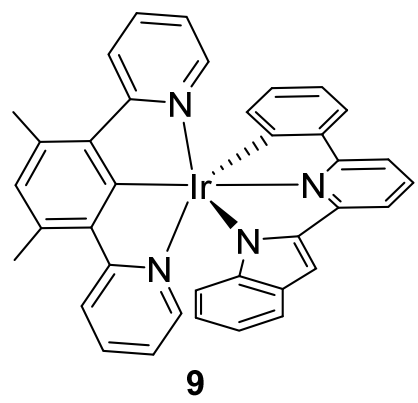

(n)

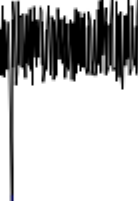

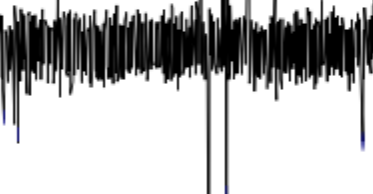

$\begin{array}{llllllllllll} & 190 & 185 & 180 & 175 & 170 & 165 & 160 & 155 & 150 & 145 & 140\end{array}$

Figure S45. ${ }^{13} \mathrm{C}\left\{{ }^{1} \mathrm{H}\right\}$-APT NMR $\left(75 \mathrm{MHz}, \mathrm{CD}_{2} \mathrm{Cl}_{2}, 298 \mathrm{~K}\right)$ spectrum of complex 9. 


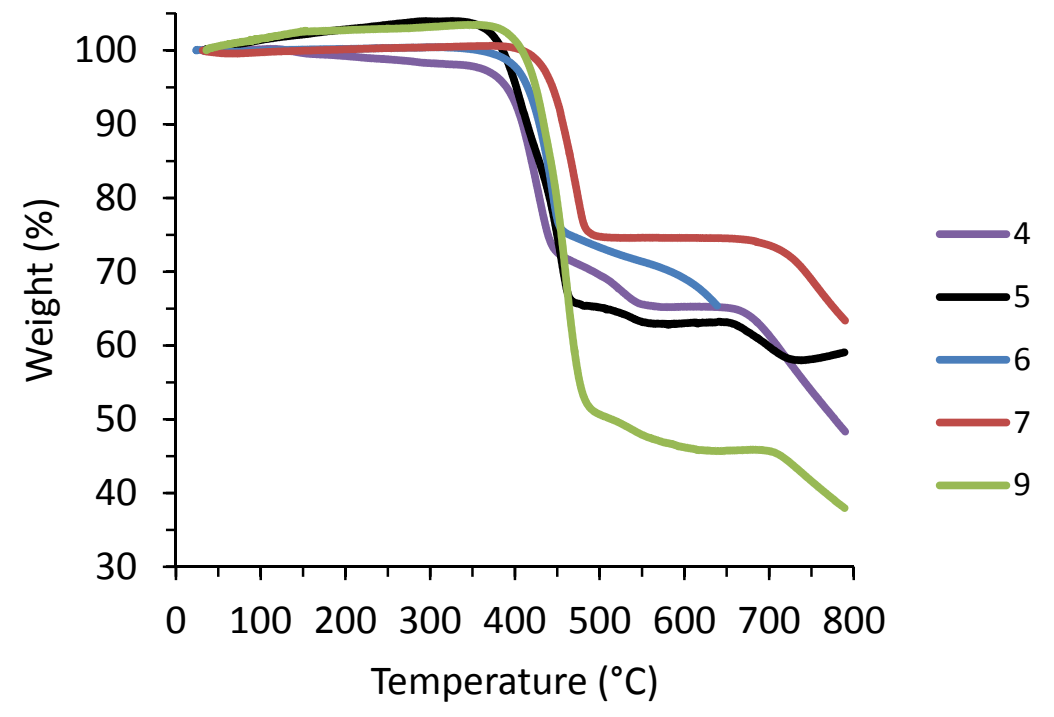

Figure S46. TGA curves of complexes 4-7 and 9. The solids were heated at $10^{\circ} \mathrm{C} / \mathrm{min}$ under nitrogen. 

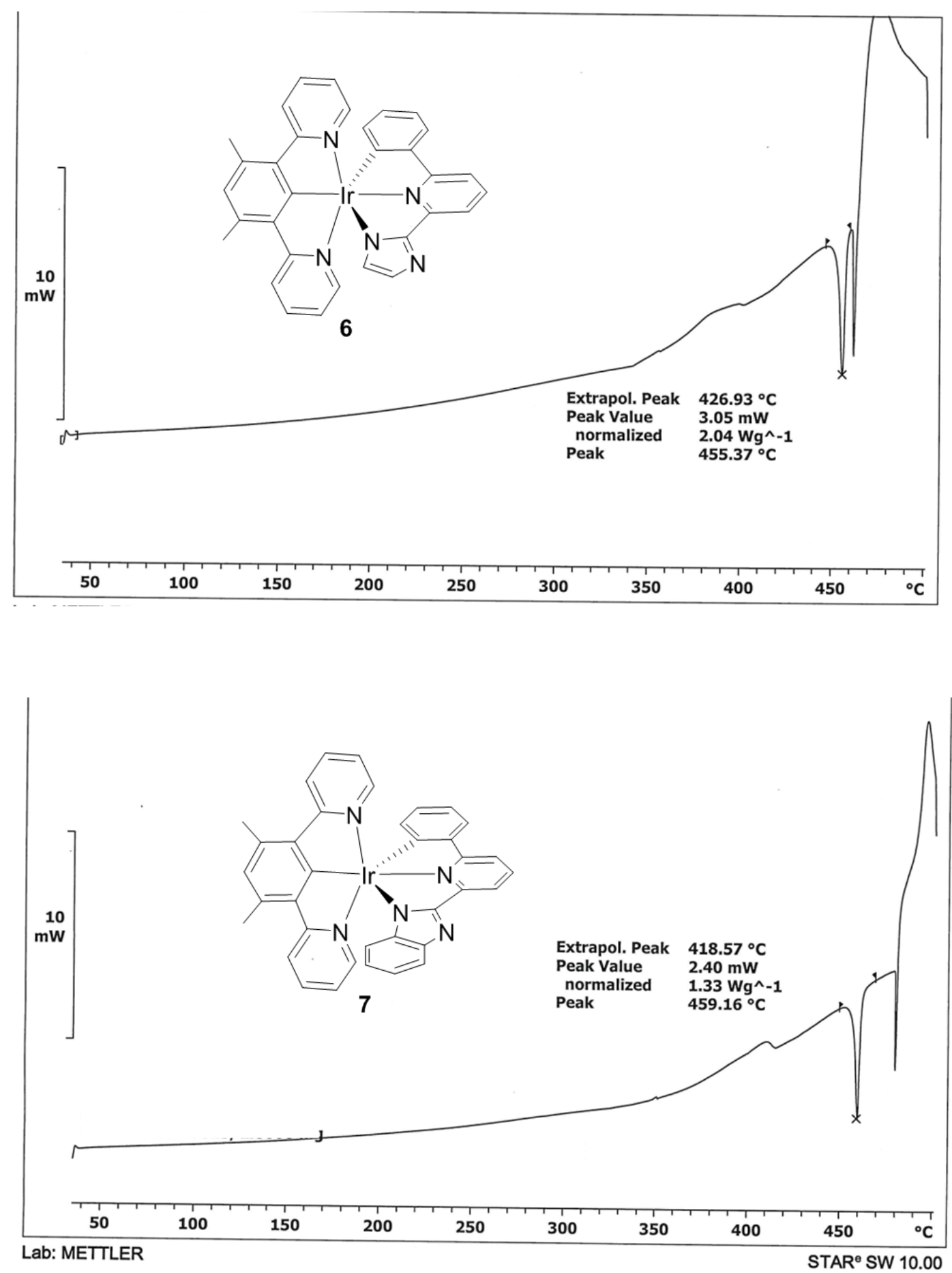

Figure S47. DSC curves of complexes 6 and 7. The solids were heated under nitrogen at $10^{\circ} \mathrm{C} / \mathrm{min}$ under nitrogen. 


\section{References}

(1) Blessing, R. H. Acta Crystallogr. 1995, A51, 33. SADABS: Area-detector absorption correction; Bruker- AXS, Madison, WI, 1996.

(2) SHELXL-2016/6. Sheldrick, G. M. A short history of SHELX. Acta Cryst. 2008, A64, 112-122.

(3) (a) Lee, C.; Yang, W.; Parr, R. G. Development of the Colle-Salvetti correlation-energy formula into a functional of the electron density. Phys. Rev. B 1988, 37, 785-789. (b) Becke, A. D. Density-functional thermochemistry. III. The role of exact exchange. J. Chem. Phys. 1993, 98, 5648-5652. (c) Stephens, P. J.; Devlin, F. J.; Chabalowski, C. F.; Frisch, M. J. Ab Initio Calculation of Vibrational Absorption and Circular Dichroism Spectra Using Density Functional Force Fields. J. Phys. Chem. 1994, 98, 1162311627.

(4) Grimme, S.; Antony, J.; Ehrlich, S.; Krieg, H. A consistent and accurate ab initio parametrization of density functional dispersion correction (DFT-D) for the 94 elements H-Pu. J. Chem. Phys. 2010, 132, 154104.

(5) Gaussian 09, Revision D.01, Frisch, M. J.; Trucks, G. W.; Schlegel, H. B.; Scuseria, G. E.; Robb, M. A.; Cheeseman, J. R.; Scalmani, G.; Barone, V.; Mennucci, B.; Petersson, G. A.; Nakatsuji, H.; Caricato, M.; Li, X., Hratchian, H. P.; Izmaylov, A. F.; Bloino, J.; Zheng, G.; Sonnenberg, J. L.; Hada, M.; Ehara, M.; Toyota, K.; Fukuda, R.; Hasegawa, J.; Ishida, M.; Nakajima, T.; Honda, Y.; Kitao, O.; Nakai, H.; Vreven, T.; Montgomery, J. A.; Peralta, Jr., J. E.; Ogliaro, F.; Bearpark, M.; Heyd, J. J.; Brothers, E.; Kudin, K. N.; Staroverov, V. N.; Keith, T.; Kobayashi, R.; Normand, J.; Raghavachari, K.; Rendell, A.; Burant, J. C.; Iyengar, S. S.; Tomasi, J.; Cossi, M.; Rega, N.; Millam, J. M.; Klene, M.; Knox, J. E.; Cross, J. B.; Bakken, V.; Adamo, C.; Jaramillo, J.; Gomperts, R.; Stratmann, R. E.; Yazyev, O.; Austin, A. J.; Cammi, R.; Pomelli, C.; Ochterski, J. W.; Martin, R. L.; Morokuma, K.; Zakrzewski, V. G.; Voth, G. A.; Salvador, P.; Dannenberg, J. J.; Dapprich, S.; Daniels, A. D.; Farkas, O.; Foresman, J. B.; Ortiz, J. V.; Cioslowski, J.; Fox, D. J. Gaussian, Inc., Wallingford CT, 2013.

(6) Andrea, D.; Haeussermann, U. M.; Dolg, M.; Stoll, H. Preuss, H. Energy-adjusted ab initio pseudopotentials for the second and third row transition elements. Theor. Chim. Acta 1990, 77, 123141.

(7) Ehlers, A. W.; Bohme, M.; Dapprich, S.; Gobbi, A.; Hollwarth, A.; Jonas, V.; Kohler, K. F.; Stegmann, R.; Veldkamp, A.; Frenking, G. A set of f-polarization functions for pseudo-potential basis sets of the transition metals Sc-Cu, Y-Ag and La-Au. Chem. Phys. Lett. 1993, 208, 111-114.

(8) (a) Hehre, W. J.; Ditchfield, R.; Pople, J. A. Self-Consistent Molecular Orbital Methods. XII. Further Extensions of Gaussian-Type Basis Sets for Use in Molecular Orbital Studies of Organic Molecules. $J$. Chem. Phys. 1972, 56, 2257-2261. (b) Francl, M. M.; Pietro, W. J.; Hehre, W. J.; Binkley, J. S.; Gordon, M. S.; DeFrees, D. J.; Pople, J. A. Self-consistent molecular orbital methods. XXIII. A polarization-type basis set for second-row elements. J. Chem. Phys. 1982, 77, 3654-3665. 
(9) Marenich, A. V.; Cramer, C. J.; Truhlar, D. G. Universal Solvation Model Based on Solute Electron Density and on a Continuum Model of the Solvent Defined by the Bulk Dielectric Constant and Atomic Surface Tensions. J. Phys. Chem. B 2009, 113, 6378-6396.

(10) O’Boyle, N. M.; Tenderholt, A. L.; Langner, K. M. cclib: A Library for Package-Independent Computational Chemistry Algorithms. J. Comput. Chem. 2008, 29, 839-845. 